ESAIM: M2AN

Vol. 40, No 3, 2006, pp. 553-595

DOI: $10.1051 / \mathrm{m} 2 \mathrm{an}: 2006022$
ESAIM: Mathematical Modelling and Numerical Analysis

www.edpsciences.org/m2an

\title{
ANALYSIS OF CRACK SINGULARITIES IN AN AGING ELASTIC MATERIAL
}

\author{
Martin Costabel $^{1}$, Monique Dauge ${ }^{1}$, Sergeï A. Nazarov ${ }^{2}$ and Jan Sokolowski ${ }^{3}$
}

\begin{abstract}
We consider a quasistatic system involving a Volterra kernel modelling an hereditarilyelastic aging body. We are concerned with the behavior of displacement and stress fields in the neighborhood of cracks. In this paper, we investigate the case of a straight crack in a two-dimensional domain with a possibly anisotropic material law. We study the asymptotics of the time dependent solution near the crack tips. We prove that, depending on the regularity of the material law and the Volterra kernel, these asymptotics contain singular functions which are simple homogeneous functions of degree $\frac{1}{2}$ or have a more complicated dependence on the distance variable $r$ to the crack tips. In the latter situation, we observe a novel behavior of the singular functions, incompatible with the usual fracture criteria, involving super polynomial functions of $\ln r$ growing in time.
\end{abstract}

Mathematics Subject Classification. 35Q72, 74D05, 74G70.

Received: March 10, 2005.

\section{INTRODUCTION}

Aging and creeping processes in an elastic body cause the elastic properties of the body to change with time, in particular by making its internal structure compatible with its deformation state by a relaxation of stresses. Such processes are relatively slow, so their modelization may neglect the dynamical effects and be done by quasistatic equations where the time $t$ is only a parameter. For a proper description of aging viscoelastic or hereditary-elastic materials, the postulated constitutive law between strains and stresses usually includes an integral operator with respect to the parameter $t$.

We do not consider here the models related to the evolution of geometry of such aging bodies, cf. [4]. So the resulting mathematical model of an aging body takes the form of a system of partial differential equations with respect to the spatial variables $x=\left(x_{1}, x_{2}\right)$, and of a perturbation in the form of an Volterra integral operator with respect to the parameter $t$.

Our paper is devoted to the mechanically relevant situation where the body contains a crack.

\footnotetext{
Keywords and phrases. Crack singularities, creep theory, Volterra kernel, hereditarily-elastic.

1 Institut de Recherche Mathématique de Rennes, Université de Rennes 1, Campus de Beaulieu, 35042 Rennes Cedex, France. Martin.Costabel@univ-rennes1.fr; Monique.Dauge@univ-rennes1.fr

2 Institute of Mechanical Engineering Problems, Laboratory of Mathematical Methods, Russian Academy of Sciences, V.O. Bol'shoi 61, 199178 St. Petersburg, Russia. serna@snark.ipme.ru

${ }^{3}$ Institut Élie Cartan, Laboratoire de Mathématiques, Université Henri Poincaré Nancy I, B.P. 239, 54506 Vandoeuvre-lès-Nancy Cedex, France. Jan.Sokolowski@iecn.u-nancy.fr
} 


\subsection{Crack in an hereditarily-elastic aging body}

We consider the case of a two-dimensional body $\Omega$ containing a crack modelized as a straight segment where traction free boundary conditions are prescribed. In the same way as in the static problems, singularities may appear in solutions of such models at crack tips. The precise knowledge of these singularities is of main interest in fracture mechanics.

In the case of the standard equations of pure linear elasticity, the asymptotics of solutions near crack tips have been known since a long time: As application of the general theory of [15] to plane angles of opening $2 \pi$, we obtain that the asymptotics at each tip are combination of homogeneous terms of the form $r^{\lambda} \mathcal{V}(\varphi)$. Here $(r, \varphi)$ are polar coordinates centered at the tip. Explicit calculations for isotropic materials prove that the $\lambda$ 's (the singularity exponents) are half-integers $\frac{1}{2}, \frac{3}{2}, \ldots$ The generalization of this result to any homogeneous anisotropic material law is due to [11] (see also [9] for a generality beyond elasticity). The square root singularity $r^{1 / 2}$ for the displacement corresponds to an unbounded stress with singularity $r^{-1 / 2}$.

Concerning homogeneous, isotropic, aging viscoelastic bodies, it is already shown, cf. [21,37] and others, that the singularities of stresses at the crack tips are of the same form as in the case of purely elastic bodies. However the stress intensity factors become time-dependent. This can be explained by an application of the so-called correspondence principle, we refer the reader to [35,37] (see also Sect. 1.3 for details).

In this paper, we make general assumptions on the instantaneous elasticity law (supposed to be anisotropic and smooth), and on the relaxation kernel (supposed to have a sectorial inhomogeneity). We prove that the standard square root singularity $r^{-1 / 2}$ of stresses is combined with a term $\Sigma$ depending in an holomorphic way on $\ln r$ ("logarithmic packet"). Such behaviour of singular solutions is shown in [29] for scalar problems, where explicit calculations are available.

In view of its relatively slow growth as $r \rightarrow 0$ (see (1.17) in the sequel) this holomorphic function $\Sigma$ leaves unchanged the power order of the stress singularity. However it makes impossible to define the stress intensity factor (see (1.18) below) and to apply stress fracture criteria.

In Section 7 certain sufficient conditions are given for the presence of such logarithmic packets. Certain fracture criteria in mechanics of cracks cannot be readily adapted to such changes of the structure of singularities. Therefore an important issue for applications is to know the precise conditions under which the square-root singularity subsists in our context of aging materials. Using the approach of [8] (see also [9, 10]), we provide in Section 6.1 a positive answer in the smooth case. More precisely, if the instantaneous Hooke's matrix and relaxation kernel are smooth functions of the spatial variables $x=\left(x_{1}, x_{2}\right)$ then the logarithms are totally absent in the asymptotic expansions. Let us point out that, however, the general anisotropic laws make the angular parts of singularities different from those obtained in the case of instantaneous elasticity problem.

There are explicit examples of hereditarily-elastic bodies for which logarithmic packets will appear at crack tips. We provide such an example, which is in a sense representative and instructive since it shows that the well established statement in the mechanical community that

"Pure square-root singularity of stresses are to be expected in the case of the invariance, with respect to the translations along the crack faces, of the instantaneous elastic moduli and of the relaxation kernel"

is not true. Indeed, we obtain logarithmic packets for instantaneously isotropic homogeneous materials provided that the crack is located at the interface between two different relaxation kernels. Such a phenomenon may be the result of aging and can be observed if, for example, the body is made of two parts, both of the same material, but starting at different moments: so the second part is added after the first one has already changed due to the aging process ( $c f$. Rem. 7.5). In our example, the instantaneous Hooke's tensor is isotropic while the relaxation kernel is also isotropic but takes different values in the upper and lower half-planes. We emphasize that the presence of logarithmic packets is ensured by a relation between the instantaneous Lamé coefficients and the coefficients of relaxation kernel. This relation resembles the famous Dundurs relation [13] providing a condition for the presence of oscillating singularities at a crack between two dissimilar isotropic elastic half-planes.

The asymptotic forms are determined thanks to a particular property of cracks in purely elastic media: in arbitrary anisotropic homogeneous body the main singularity exponent of stresses is always equal to $-1 / 2$, 


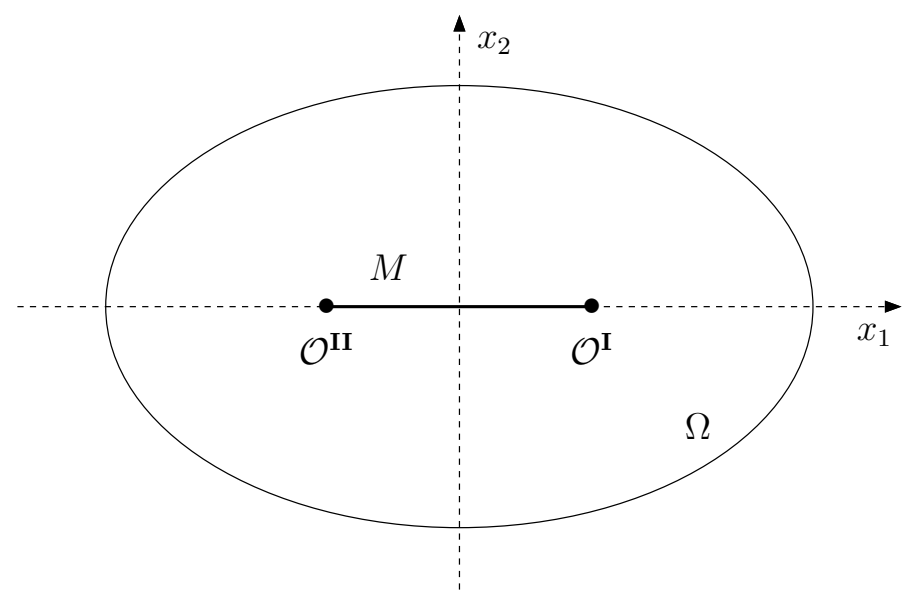

Figure 1. The domain with crack.

therefore independent of time. Our mathematical framework does not apply to the case of time-dependent exponents, thus discards the case of aging media in reference domains with angles different from $2 \pi$ (V-notch). There exist results $[5-7,22,23]$ on asymptotic behaviour of stresses in hereditarily-elastic aging media for small times $t \rightarrow 0$ and large times $t \rightarrow \infty$, but such results are unfortunately not sufficiently precise to be used in fracture mechanics. The asymptotic structure of physical fields for time-dependent exponents still remains an open problem.

\subsection{Mathematical formulation of the problem}

In the framework of creep theory [2,4], we consider a hereditarily-elastic aging, anisotropic, nonhomogeneous two-dimensional body $\Omega$ with a straight crack: $\Omega=\Omega_{0} \backslash M$ where $\Omega_{0}$ is a smooth domain and $M$ represents the crack

$$
M=\left\{x=\left(x_{1}, x_{2}\right):\left|x_{1}\right| \leq 1, x_{2}=0\right\} \subset \Omega_{0} .
$$

We denote the crack tips $(\mathbf{l}, 0)$ and $(-\mathbf{l}, 0)$ by $\mathcal{O}^{\mathbf{I}}$ and $\mathcal{O}^{\mathbf{I I}}$, respectively, and the crack surfaces by $M^{ \pm}$. Thus the boundary $\partial \Omega$ is the union of the exterior boundary $\partial \Omega_{0}=: \Gamma$ and of the crack surfaces $M^{ \pm}$. Let $n$ stand for the unit outward normal vector (column) to the boundary $\partial \Omega$. On $M^{ \pm}$, we have $n=\mp \mathbf{e}_{2}$ where $\mathbf{e}_{2}$ denotes the second basis vector $(0,1)^{\top}$. Note that the normal is not defined at the tips $\mathcal{O}^{\mathfrak{i}}$ (here and further $\mathfrak{i}$ stands for either $\mathbf{I}$ or II). In the sequel we treat dimensionless coordinates and, by rescaling, we achieve $\mathbf{l}=1$.

Besides Cartesian coordinates, we need polar coordinates $\left(r_{\mathfrak{i}}, \varphi_{\mathfrak{i}}\right)$ attached to each crack tip $\mathcal{O}^{\mathfrak{i}}$. Of course $r_{\mathfrak{i}}=\left|x-\mathcal{O}^{\mathfrak{i}}\right|$, and we choose $\varphi_{\mathfrak{i}}$ so that the crack $M$ is included in the lines $\varphi_{\mathfrak{i}}= \pm \pi$.

Equilibrium equations and boundary conditions are written in matrix form as follows:

$$
\left\{\begin{aligned}
D\left(-\nabla_{x}\right)^{\top} \sigma(u ; x, t) & =f(x, t), & & x \in \Omega=\Omega_{0} \backslash M \\
D(n(x))^{\top} \sigma(u ; x, t) & =g(x, t), & & x \in \partial \Omega \backslash\left\{\mathcal{O}^{\mathbf{I}} \cup \mathcal{O}^{\mathbf{I I}}\right\} .
\end{aligned}\right.
$$

Here $f=\left(f_{1}, f_{2}\right)^{\top}$ and $g=\left(g_{1}, g_{2}\right)^{\top}$ are the vectors of volume forces and tractions, respectively, in the form of column vectors ( $\top$ means transposition) and $\sigma(u ; x, t)$ stands for the vector of stresses at the point $x$ and at the time $t$, evaluated for the displacement vector $u=\left(u_{1}, u_{2}\right)^{\top}$. Moreover, $D\left(\nabla_{x}\right)$ denotes the $3 \times 2$-matrix of differential operators,

$$
D\left(\nabla_{x}\right)^{\top}=\left(\begin{array}{ccc}
\partial_{1} & 0 & 2^{-1 / 2} \partial_{2} \\
0 & \partial_{2} & 2^{-1 / 2} \partial_{1}
\end{array}\right), \quad \nabla_{x}=\left(\begin{array}{c}
\partial_{1} \\
\partial_{2}
\end{array}\right), \quad \partial_{j}=\frac{\partial}{\partial x_{j}}, \quad j=1,2 .
$$


The column $\varepsilon=\left(\varepsilon_{11}, \varepsilon_{22}, 2^{1 / 2} \varepsilon_{12}\right)^{\top}$ of height 3 ,

$$
\varepsilon(u ; x, t)=D\left(\nabla_{x}\right) u(x, t),
$$

denotes the vector of strains. The factors $2^{-1 / 2}$ are introduced into (1.3) in order to equalize natural norms of the strain column above and the usual norm of strain tensor of rank 2.

In an hereditary-elastic aging body, $\sigma$ is determined according to the constitutive law

$$
\sigma(u ; x, t)=A(x, t) D\left(\nabla_{x}\right) u(x, t)+\int_{0}^{t} B(x, t, \tau) D\left(\nabla_{x}\right) u(x, \tau) \mathrm{d} \tau .
$$

Here, the $3 \times 3$-matrix functions $A$ and $B$ are the Hooke matrix and the relaxation kernel, respectively. They are symmetric by definition and, furthermore, $A(x, t)$ is supposed to be positive definite:

$$
\xi^{\top} A(x, t) \xi \geq c_{A}|\xi|^{2} \quad \forall \xi \in \mathbb{R}^{3}, \quad x \in \Omega_{0}, \quad t \in(0, T)
$$

with a positive constant $c_{A}$. We make the following smoothness assumption on the coefficients of $A$ : for a fixed integer $\ell>0$,

$$
\left|\nabla_{x}^{j} A(x, t)\right| \leq c_{A}^{\prime}, \quad j=0, \ldots, \ell, \quad x \in \bar{\Omega}_{0}, \quad \text { for a.e. } t \in(0, T),
$$

where $\nabla_{x}^{j} w$ denotes the set of all derivatives of order $j$ of the function $w$. In other words, entries of $A$ are $\ell$-times continuously differentiable functions in $x \in \bar{\Omega}_{0}$ (in the body without crack) and measurable bounded functions in $t \in[0, T]$.

Since the relaxation kernel can possess a cylindrical anisotropy (cf. [2]) in the vicinity of the crack tips, we assume a weaker regularity hypothesis for the matrix $B$. Let $\rho$ be a positive function on $\bar{\Omega}_{0} \backslash\left\{\mathcal{O}^{\mathbf{I}} \cup \mathcal{O}^{\mathbf{I I}}\right\}$ which coincides with $r_{\mathfrak{i}}$ in a neighbourhood of $O^{\mathfrak{i}}$, e.g., $\rho=\min \left\{1, r_{\mathbf{I}}, r_{\mathbf{I I}}\right\}$. The relaxation kernel $B$ satisfies the following weighted condition:

$$
\left|\nabla_{x}^{j} B(x, t, \tau)\right| \leq c_{B} \rho(x)^{-j}, \quad j=0, \ldots, \ell, \quad x \in \Omega, \quad \text { for a.e. }(t, \tau) \in \mathcal{T}(T),
$$

with the triangle

$$
\mathcal{T}(T):=\{(t, \tau): t \in(0, T), \tau \in(0, t)\} .
$$

Assumption (1.7) is sufficient for the existence of a solution $u$ to problem (1.2)-(1.4).

However the determination of asymptotic properties of the solution $u(x, t)$ requires stronger hypotheses on the relaxation kernel. We assume that at each crack tip $\mathcal{O}^{\mathfrak{i}}, \mathfrak{i}=\mathbf{I}, \mathbf{I I}, B(x, \cdot, \cdot)$ stabilizes as $r_{\mathfrak{i}} \rightarrow 0$ towards a matrix $B^{\mathfrak{i}}\left(\varphi_{\mathfrak{i}}, \cdot, \cdot\right)$ which only depend on the angular variable $\varphi_{\mathbf{i}} \in(-\pi, \pi)$ : for a suitable stabilization rate $\delta_{B}>\frac{1}{2}$

$$
\left|\nabla_{x}^{j}\left(B(x, t, \tau)-B^{\mathfrak{i}}\left(\varphi_{\mathfrak{i}}, t, \tau\right)\right)\right| \leq c_{B}^{\prime} r_{\mathfrak{i}}^{-j+\delta_{B}}, \quad j=0, \ldots, \ell, \quad x \in \Omega, \quad \text { for a.e. }(t, \tau) \in \mathcal{T}(T)
$$

where entries of $B^{\mathfrak{i}}$ are $l$-times continuously differentiable in $\varphi_{\mathfrak{i}} \in[-\pi, \pi]$ and bounded in $(t, \tau) \in \mathcal{T}(T)$. Thus $B^{\mathfrak{i}}$ may have a jump through the crack $M$. We note that condition (1.9) implies condition (1.7). Moreover, if $B$ satisfies a smoothness condition like (1.6), namely

$$
\left|\nabla_{x}^{j} B(x, t, \tau)\right| \leq c_{B}, \quad j=0, \ldots, \ell, \quad x \in \bar{\Omega}_{0}, \quad \text { for a.e. }(t, \tau) \in \mathcal{T}(T),
$$

condition (1.9) is obviously fulfilled with $B^{\mathfrak{i}}:=B\left(\mathcal{O}^{\mathfrak{i}}\right)$, which is independent of $\varphi_{\mathfrak{i}}$.

We introduce the following notations for the second order partial differential operators intervening in problem (1.2)-(1.4): for the instantaneous operators, we denote

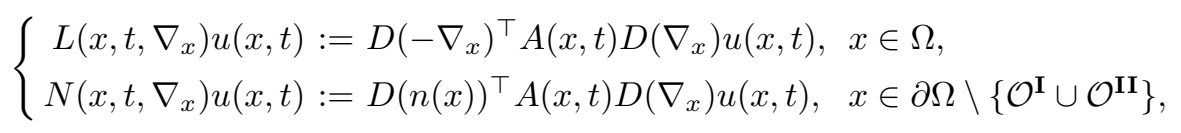


whereas the differential operators associated with the relaxation kernel are denoted by

$$
\begin{cases}P\left(x, t, \tau, \nabla_{x}\right):=D\left(-\nabla_{x}\right)^{\top} B(x, t, \tau) D\left(\nabla_{x}\right), & x \in \Omega, \\ Q\left(x, t, \tau, \nabla_{x}\right):=D(n(x))^{\top} B(x, t, \tau) D\left(\nabla_{x}\right), & x \in \partial \Omega \backslash\left\{\mathcal{O}^{\mathbf{I}} \cup \mathcal{O}^{\mathbf{I I}}\right\} .\end{cases}
$$

With these notations problem (1.2)-(1.4) can be written in the condensed form,

$$
\begin{aligned}
& \{L(t), N(t)\} u(t)+\int_{0}^{t}\{P(t, \tau), Q(t, \tau)\} u(\tau) \mathrm{d} \tau=\{f(t), g(t)\} \\
& \quad \text { on } \Omega \times\left(\partial \Omega \backslash\left\{\mathcal{O}^{\mathbf{I}} \cup \mathcal{O}^{\mathbf{I I}}\right\}\right), \text { for a.e. } t \in(0, T)
\end{aligned}
$$

without any explicit reference to the dependence on the variable $x$ of functions $u, f, g$ and omitting the differential operator $\nabla_{x}$.

\subsection{Main results and structure of the paper}

Relying on the formulation (1.2)-(1.4) of the creep problem, we study the behavior of physical fields $u(x, t)$ and $\sigma(u ; x, t)$ near the crack tips, i.e. as $r_{\mathfrak{i}} \rightarrow 0, \mathfrak{i}=\mathbf{I}, \mathbf{I I}$. In particular we give a precise description of the singularities of the stress field.

The classical result of the isotropic elasticity theory, which serves as a base of fracture mechanics, leads to singularities of square-root type at the crack tips. It is well known (see e.g., [3,21,36,37], and others) that the same type of singularities occur in the creep theory for instantaneously isotropic homogeneous materials with the isotropic relaxation kernel, i.e.,

$$
\begin{gathered}
A(t)=\left(\begin{array}{ccc}
\lambda(t)+2 \mu(t) & \lambda(t) & 0 \\
\lambda(t) & \lambda(t)+2 \mu(t) & 0 \\
0 & 0 & 2 \mu(t)
\end{array}\right) \\
B(t, \tau)=\left(\begin{array}{ccc}
\lambda^{\prime}(t, \tau)+2 \mu^{\prime}(t, \tau) & \lambda^{\prime}(t, \tau) & 0 \\
\lambda^{\prime}(t, \tau) & \lambda^{\prime}(t, \tau)+2 \mu^{\prime}(t, \tau) & 0 \\
0 & 0 & 2 \mu^{\prime}(t, \tau)
\end{array}\right) ;
\end{gathered}
$$

here $\lambda(t) \geq 0, \mu(t)>0$ are the Lamé coefficients. In the cited works several different mathematical approaches are used. However, at the final stage, explicitly or implicitly, the main role is played by the correspondence principle (cf. $[32,35])$, which states that

"The stress state of a plane homogeneous and isotropic body is independent of the Poisson ratio $\sigma=\lambda[2(\lambda+\mu)]^{-1}$ in the absence of body forces, in the case of selfequilibrium of exterior tractions applied on each connected component of the boundary."

For problem (1.2)-(1.4) with the matrices defined by (1.14), the Poisson ratio can be interpreted as the composition of Volterra integral operators,

$$
z \mapsto \lambda(t) z(t)+\int_{0}^{t} \lambda^{\prime}(t, \tau) z(t, \tau) \mathrm{d} \tau, \quad z \mapsto \mu(t) z(t)+\int_{0}^{t} \mu^{\prime}(t, \tau) z(t, \tau) \mathrm{d} \tau
$$

which allows, in principle, to express the singular solution of the creep problem by means of the singular solutions to the elasticity system. However, it leads also to some difficulties connected with the fact that the operators defined by (1.15) do not commute. We refer the reader to [37] for details.

We show in the sequel that for an arbitrary anisotropic aging hereditarily-elastic medium, the stress singularities might change and depend holomorphically on the logarithm of the polar coordinate $r_{\mathfrak{i}}$, 
so that, for any $\varepsilon \in(0,1 / 2)$,

$$
\sigma(u ; x, t)=r_{\mathfrak{i}}^{-1 / 2} \Sigma^{\mathfrak{i}}\left(\ln r_{\mathfrak{i}}, \varphi_{\mathfrak{i}}, t\right)+O\left(r_{\mathfrak{i}}^{\varepsilon-1 / 2}\right), \quad x \rightarrow \mathcal{O}^{\mathfrak{i}} .
$$

As we prove in Theorem 6.4 , for any $\varphi \in[-\pi, \pi]$ and a.e. $t \in[0, T]$ the following estimate is valid:

$$
\left|\Sigma^{\mathbf{i}}(\ln r, \varphi, t)\right| \leq c \exp \left(d_{1} t+d_{2} \sqrt{t|\ln r|}\right)
$$

where $c, d_{1}, d_{2}$ are positive constants. Thus, the function $z \mapsto \Sigma^{\mathfrak{i}}(-z, \varphi, t)$ grows faster than any polynomial $z^{N}, N>0$, but slower compared to any exponential function $\exp (\varepsilon z), \varepsilon>0$. In this way, the power order $r_{\mathfrak{i}}^{-1 / 2}$ of the stress singularity is maintained in the creep theory as well. However, in opposition to pure elasticity, the product

$$
r_{\mathfrak{i}}^{1 / 2} \sigma(u ; x, t)
$$

with fixed $\varphi$ and $t$ may have no limit as $r_{\mathfrak{i}} \rightarrow 0$. Therefore, the usual definition of the stress intensity factors, referring to the limit of (1.18) at $\varphi_{\mathfrak{i}}=0$ with $r_{\mathfrak{i}} \rightarrow 0$, do not make sense any more, which invalidates certain fracture criteria. In this context, the conditions on the model which assure the absence of logarithmic packets are of importance, in particular for the applicability of classical fracture criteria to the creep problems. We prove that if $B^{\mathfrak{i}}$ in (1.9) does not depend on $\varphi_{\mathfrak{i}}$, then the angular part $\Sigma^{\mathfrak{i}}$ in (1.16) does not contain $\ln r_{\mathfrak{i}}$ at all. At the same time, we point out certain sufficient conditions which guarantee the appearance of logarithmic packets in (1.16).

The paper is organized as follows. In Section 2, the classical method for Volterra equations is applied to obtain the existence of a weak solution to problem (1.13) defined by (1.2)-(1.4). In Section 3, we prove a basic regularity result in the form of an exponential estimate in $t$ for the solution $u(x, t)$ in some suitable weighted Sobolev spaces with respect to the spatial variable $x$. These weighted spaces contain the standard singularities in $r^{-1 / 2}$ of the stresses, thus the displacements described by these spaces are not $\mathcal{C}^{1}$ up to the crack tips, in general.

In the next two Sections 4 and 5, we prepare the material for the proof (done in Sect. 6) of a splitting of the time dependent solution $u(x, t)$ into a regular part and a singular part made of two logarithmic packets. Sections 4 and 5 are devoted to the instantaneous problems, i.e., the problems where the time $t$ is simply a frozen parameter in the Hooke matrix $A$, and the Volterra kernel is absent (or considered as an independent right hand side through a bootstrap procedure).

We gather in Section 4 classical material related to corner asymptotics for elasticity solutions, namely the Mellin transformation and the main singularities. In Section 5 we combine the asymptotics for the instantaneous problem with the bootstrap procedure. This results in new original estimates on finite logarithmic packets of arbitrary length. Relying on the approach initiated in [8], we also investigate the situation of a stabilized kernel $B^{\mathfrak{i}}$ independent of the angular variable: we prove in this case that we are staying in the same class of fields with separate asymptotics without logarithms, along the whole bootstrap procedure. Finally in Section 6, we come back to the time dependent problem and prove the results already mentioned above. We end by a discussion of the logarithmic packets in Section 7.

\section{Existence of solutions and exponential estimates}

In this section, we use a standard method for Volterra equations (see e.g. $[14,18]$ ) to derive existence and estimates for a finite energy solution to problem (1.13).

We will use everywhere the following generic notation: for a Banach space $\mathcal{B}$ with norm $\|\cdot\|_{\mathcal{B}}$, let $L_{\infty}(0, T ; \mathcal{B})$ denote the space of abstract functions in the interval $(0, T)$ with values in the space $\mathcal{B}$, equipped with the norm

$$
\|U ; T\|_{\mathcal{B}}=\operatorname{ess} \sup \left\{\|U(t)\|_{\mathcal{B}}: t \in(0, T)\right\} .
$$




\subsection{General method for Volterra equations}

For the convenience of the reader, we provide the existence and uniqueness result for an abstract Volterra equation

$$
\mathfrak{A}(t) \mathfrak{u}(t)+\int_{0}^{t} \mathfrak{B}(t, \tau) \mathfrak{u}(\tau) \mathrm{d} \tau=\mathfrak{f}(t), \text { for a.e. } t \in(0, T),
$$

where $\mathfrak{A}(t): \mathfrak{D} \rightarrow \mathfrak{R}$ and $\mathfrak{B}(t, \tau): \mathfrak{D} \rightarrow \mathfrak{R}$ are families of continuous operators between the Banach spaces $\mathfrak{D}$ and $\mathfrak{R}$. We assume moreover that the operators $\mathfrak{A}(t)$ are invertible and that the inverse operators $\mathfrak{A}(t)^{-1}$ and the operators $\mathfrak{B}(t, \tau)$ are measurable and bounded for $t \in(0, T)$ and $(t, \tau)$ in the triangle $\mathcal{T}(T)$ (1.8), respectively.

Theorem 2.1. For any $\mathfrak{f} \in L_{\infty}(0, T ; \mathfrak{R})$, there exists a unique solution $\mathfrak{u} \in L_{\infty}(0, T ; \mathfrak{R})$ of equation (2.1). Moreover there holds the estimate

$$
\|\mathfrak{u} ; t\|_{\mathfrak{D}} \leq c_{0} \mathrm{e}^{\delta_{0} t}\|\mathfrak{f} ; t\|_{\mathfrak{R}}, \quad \text { for } \quad \text { a.e. } t \in(0, T),
$$

for any $c_{0}$ and $\delta_{0}$ such that

$$
c_{0} \geq \underset{t \in(0, T)}{\operatorname{ess} \sup }\left\|\mathfrak{A}(t)^{-1}\right\|_{\mathfrak{R} \rightarrow \mathfrak{D}} \quad \text { and } \quad \delta_{0} \geq \operatorname{ess~sup}_{(t, \tau) \in \mathcal{T}(T)}\left\|\mathfrak{A}(t)^{-1} \mathfrak{B}(t, \tau)\right\|_{\mathfrak{D} \rightarrow \mathfrak{D}} .
$$

Proof.

(i) Existence. We search the solution in the form of the series

$$
\mathfrak{u}(t)=\sum_{k=0}^{\infty} \mathfrak{u}^{k}(t)
$$

where for $k \geq 0$

$$
\mathfrak{A}(t) \mathfrak{u}^{k}(t)=\delta_{0, k} \mathfrak{f}(t)-\int_{0}^{t} \mathfrak{B}(t, \tau) \mathfrak{u}^{k-1}(\tau) \mathrm{d} \tau .
$$

Here we have set $\mathfrak{u}^{-1}=0$ and $\delta_{i, k}$ is the Kronecker symbol. Let us prove the estimate

$$
\left\|\mathfrak{u}^{k} ; t\right\|_{\mathfrak{D}} \leq c_{0}(k !)^{-1}\left(\delta_{0} t\right)^{k}\|\mathfrak{f} ; t\|_{\mathfrak{R}} \text {, for a.e. } t \in(0, T),
$$

by induction. Since for $k=0$ estimate (2.5) is evident, it suffices to show the estimate with $k=K>0$ can be deduced from the the estimates for $k \leq K-1$. To this end, in view of $(2.3)$, we have

$$
\begin{aligned}
\left\|\mathfrak{u}^{K} ; t\right\|_{\mathcal{D}} \leq \delta_{0} \int_{0}^{t}\left\|\mathfrak{u}^{K-1}(\tau)\right\|_{\mathfrak{D}} \mathrm{d} \tau & \leq \delta_{0} \int_{0}^{t}\left\|\mathfrak{u}^{K-1} ; \tau\right\|_{\mathfrak{D}} \mathrm{d} \tau \\
& \leq c_{0} \delta_{0}^{K} \int_{0}^{t} \frac{\tau^{K-1}}{(K-1) !}\|\mathfrak{f} ; \tau\|_{\mathfrak{R}} \mathrm{d} \tau \leq c_{0} \frac{\left(\delta_{0} t\right)^{K}}{K !}\|\mathfrak{f} ; t\|_{\mathfrak{R}} .
\end{aligned}
$$

Estimate (2.5) implies the convergence of series (2.4) as well as estimate (2.2) which follows by the Taylor formula for the exponential function $t \mapsto \mathrm{e}^{\delta_{0} t}$.

(ii) Uniqueness. If $\mathfrak{u}$ is a solution of the homogeneous equation (2.1) and $\mathfrak{u}(t)=0$ for a.e. $t \in\left(0, t_{0}\right)$, then

$$
\|\mathfrak{u} ; t\|_{\mathfrak{D}} \leq \delta_{0}\left(t-t_{0}\right)\|\mathfrak{u} ; t\|_{\mathfrak{D}} \quad \forall t \geq t_{0},
$$

and therefore, $\mathfrak{u}(t)=0$ for a.e. $t \in\left(0, t_{1}\right)$ with any $t_{1}<t_{0}+\delta_{0}^{-1}$. The proof is completed. 


\subsection{Energy solutions}

We come back to creep problem (1.2)-(1.4). We impose the following integrability conditions on the data:

$$
f \in L_{\infty}\left(0, T ; L_{2}(\Omega)\right)^{2}, \quad g \in L_{\infty}\left(0, T ; L_{2}(\partial \Omega)\right)^{2},
$$

together with the compatibility conditions (selfequilibrium of the loading for a.e. $t$ )

$$
(f(\cdot, t), v)_{\Omega}+(g(\cdot, t), v)_{\partial \Omega}=0 \quad \forall v \in \mathcal{R}, \quad \text { a.e.t } \in(0, T),
$$

where $\mathcal{R}=\left\{\left(c_{1}-c_{0} x_{2}, c_{2}+c_{0} x_{1}\right): c_{q} \in \mathbb{R}\right\}$ denotes the linear space of rigid motions. In $(2.7)(\cdot, \cdot)_{\Xi}$ is the scalar product in the space $L_{2}(\Xi)$, the same symbol is used for the scalar product of vector functions and we denote for simplicity $L_{2}(\Xi)^{n}=L_{2}\left(\Xi ; \mathbb{R}^{n}\right)$ for any $n \in \mathbb{N}$. To ensure uniqueness of the solution, the following normalization condition is imposed:

$$
(u(\cdot, t), v)_{\Omega}=0 \quad \forall v \in \mathcal{R} \quad \text { for a.e. } t \in(0, T) .
$$

Theorem 2.2. Under conditions (2.6)-(2.8), there exists a unique solution

$$
u \in L_{\infty}\left(0, T ; H^{1}(\Omega)\right)^{2},
$$

to problem (1.2)-(1.4). Moreover u satisfies the estimate

$$
\|u ; t\|_{H^{1}(\Omega)} \leq C \mathrm{e}^{\delta t}\left\{\|f ; t\|_{L_{2}(\Omega)}+\|g ; t\|_{L_{2}(\partial \Omega)}\right\}
$$

for $t \in(0, T)$ and some positive constants $C$ and $\delta$.

Proof. Let us set

$$
\mathfrak{D}=\left\{u \in H^{1}(\Omega)^{2}: u \text { satisfies orthogonality condition }(2.8)\right\} .
$$

and let $\mathfrak{R}$ be the dual space of $\mathfrak{D}^{\prime}$ for the extension of the $L^{2}$ duality. Condition (1.5) together with the Korn inequality

$$
\|u\|_{H^{1}(\Omega)} \leq C_{\Omega}\left\|D\left(\nabla_{x}\right) u\right\|_{L_{2}(\Omega)} \quad \forall u \in \mathfrak{D}
$$

(see, e.g. $[16,33])$, implies that the operators $\mathfrak{A}(t)$ defined by

$$
\mathfrak{A}(t)(u)=\left(v \longmapsto \int_{\Omega}\left[D\left(\nabla_{x}\right) v(x)\right]^{\top} A(x, t) D\left(\nabla_{x}\right) u(x) \mathrm{d} x\right)
$$

are isomorphisms from $\mathfrak{D}$ onto $\mathfrak{R}$ with bounded inverses $\mathfrak{A}(t)^{-1}$. The operators

$$
\mathfrak{B}(t, \tau)(u)=\left(v \longmapsto \int_{\Omega}\left[D\left(\nabla_{x}\right) v(x)\right]^{\top} B(x, t, \tau) D\left(\nabla_{x}\right) u(x) \mathrm{d} x\right),
$$

are well defined from $\mathfrak{D}$ into $\mathfrak{R}$ because, as $D\left(\nabla_{x}\right) v(x)=0$ for all rigid motions $v \in \mathcal{R}$, cf. the structure of matrix $D\left(\nabla_{x}\right)$ in $(1.3)$ :

$$
\int_{\Omega}\left[D\left(\nabla_{x}\right) v(x)\right]^{\top} B(x, t, \tau) D\left(\nabla_{x}\right) u(x) \mathrm{d} x=0 \quad \forall u \in \mathfrak{D}, \quad v \in \mathcal{R} .
$$

Let the right hand side $\mathfrak{f}(t)$ be defined as

$$
\mathfrak{f}(t)=\left(v \longmapsto \int_{\Omega} v(x)^{\top} f(x, t) \mathrm{d} x+\int_{\partial \Omega} v(x)^{\top} g(x, t) \mathrm{d} \sigma\right) .
$$


It is clear that conditions $(2.6)-(2.7)$ imply that $\mathfrak{f}$ belongs to $L_{\infty}(0, T ; \mathfrak{R})$. Therefore Theorem 2.1 can be applied: it yields existence and uniqueness of a solution for problem (1.2)-(1.4), and estimates (2.10).

Remark 2.3. For the conclusions of Theorem 2.2, weaker hypotheses on the matrix coefficients $A(x, t)$ and $B(x, t, \tau)$ than those formulated in (1.6) and (1.7) would be sufficient. One only needs that $A$ and $A^{-1}$ are uniformly bounded on $\Omega \times(0, T)$ and that $B$ is uniformly bounded on $\Omega \times \mathcal{T}(T)$. In particular, piecewise constant $B$ on the half planes $\pm x_{2} \geq 0$ is admissible.

\section{Regularity of SOlutions}

We have just seen that a direct application of Theorem 2.1 gives the existence of a finite energy solution $u(x, t)$ for problem (1.13). The same statement also allows to prove regularity results for $u$ if the data $\{f, g\}$ are more regular and if we know suitable couples of spaces $(\mathfrak{D}, \mathfrak{R})$ for which Theorem 2.1 applies.

But the presence of the crack induces the appearance of singularities for the solutions of the instantaneous problems $\mathfrak{A}(t)$. The first ones of these singularities have the form

$$
V: x \longmapsto \sqrt{r_{\mathfrak{i}}} \mathcal{V}_{\mathbf{i}}\left(\varphi_{\mathfrak{i}}\right),
$$

with a smooth function $\mathcal{V}_{\mathfrak{i}}$ of the angular variable $\varphi_{\mathfrak{i}}$. Therefore, the space $\mathfrak{D}$ should contain such functions. The use of standard Sobolev spaces is very limitative: we could take $H^{s}(\Omega)$ for $s<\frac{3}{2}$ only. A much more appropriate option consists in choosing weighted Sobolev spaces of Kondrat'ev type [15]. These spaces will also serve to set the stronger assumptions on the data which we will use on the rest of the paper in view of the investigation of the leading crack singularities.

\subsection{Weighted Sobolev spaces}

Let $\mathfrak{C}_{0}^{\infty}(\Omega)$ be the space of the functions from $\mathcal{C}^{\infty}\left(\Omega \cup \partial \Omega_{0}\right)$ vanishing near the crack tips $\mathcal{O}^{\mathfrak{i}}$ and being smooth up to the crack surfaces, i.e., jumps on $M$ are allowed. With this notation, the Kondrat'ev space $V_{\beta}^{m}(\Omega)$ is defined for $\beta \in \mathbb{R}$ and $m \in \mathbb{N}_{0}=\{0,1, \ldots\}$ as the completion of the space of smooth functions $\mathfrak{C}_{0}^{\infty}(\Omega)$ with respect to the norm

$$
\|w\|_{V_{\beta}^{m}(\Omega)}=\left(\sum_{j=0}^{m}\left\|\rho^{\beta-m+j} \nabla_{x}^{j} w\right\|_{L_{2}(\Omega)}^{2}\right)^{1 / 2},
$$

where $\rho=\min \left\{1, r_{\mathbf{I}}, r_{\mathbf{I I}}\right\}$ is the same weight function as in (1.7).

We also need trace spaces for $m \geq 1$. Note that the boundary $\partial \Omega$ is the union of the external boundary $\partial \Omega_{0}$ and the two sides $M^{ \pm}$of the crack. Thus a trace $\psi$ on $\partial \Omega$ is equivalent to the data of

$$
\psi^{0}=\left.\psi\right|_{\partial \Omega_{0}} \quad \text { and } \quad \psi^{ \pm}=\left.\psi\right|_{M^{ \pm}} .
$$

Let us denote the trace operator $\left.w \mapsto w\right|_{\partial \Omega}$ by $\boldsymbol{\Gamma}_{0}$, the trace operator on $\partial \Omega_{0}$ by $\boldsymbol{\Gamma}_{0}^{0}$ and the trace operators on $M^{ \pm}$by $\boldsymbol{\Gamma}_{0}^{ \pm}$.

Since $\partial \Omega_{0}$ is disjoint from the crack, it is obvious that $\boldsymbol{\Gamma}_{0}^{0}\left(V_{\beta}^{m}(\Omega)\right)$ coincides with the standard trace space $H^{m-1 / 2}\left(\partial \Omega_{0}\right)$. On $M^{+}$and $M^{-}$, we introduce the spaces $V_{\beta}^{m-1 / 2}\left(M^{ \pm}\right)$as the closure of $\mathcal{C}_{0}^{\infty}\left(M^{ \pm}\right)$for the norm (3.2):

$$
\begin{aligned}
\|\psi\|_{V_{\beta}^{m-1 / 2}\left(M^{ \pm}\right)} & =\left\{\sum_{k=0}^{m-1}\left\|\rho^{\beta-\left(m-\frac{1}{2}\right)+k} \partial_{1}^{k} \psi\right\|_{L_{2}(M)}^{2}+I_{m, \beta}^{ \pm}(\psi)\right\}^{1 / 2} \text { with } \\
& I_{m, \beta}^{ \pm}(\psi)=\int_{-1}^{1} \int_{-1}^{1}\left|\rho\left(x_{1}\right)^{\beta} \partial_{1}^{m-1} \psi\left(x_{1}, \pm 0\right)-\rho\left(y_{1}\right)^{\beta} \partial_{1}^{m-1} \psi\left(y_{1}, \pm 0\right)\right|^{2} \frac{\mathrm{d} x_{1} \mathrm{~d} y_{1}}{\left|x_{1}-y_{1}\right|^{2}}
\end{aligned}
$$


Here $\partial_{1}^{k}$ denotes the partial derivative of order $k$ in $x_{1}$, i.e., along the crack. As shown e.g. in [17] the space $V_{\beta}^{m-1 / 2}\left(M^{+}\right)$coincides with the trace space $\boldsymbol{\Gamma}_{0}^{+}\left(V_{\beta}^{\ell}(\Omega)\right)$, and the same for $\boldsymbol{\Gamma}_{0}^{-}$.

Finally we choose to denote by $V_{\beta}^{m-1 / 2}(\partial \Omega)$ the direct sum

$$
V_{\beta}^{m-1 / 2}(\partial \Omega):=H^{m-1 / 2}\left(\Gamma_{0}\right) \oplus V_{\beta}^{m-1 / 2}\left(M^{+}\right) \oplus V_{\beta}^{m-1 / 2}\left(M^{-}\right) .
$$

Thanks to the density of smooth functions which are zero on the crack tips, we can show that $\boldsymbol{\Gamma}_{0}\left(V_{\beta}^{\ell}(\Omega)\right)$ coincides with $V_{\beta}^{m-1 / 2}(\partial \Omega)$ algebraically and topologically. In other words

$$
\psi \in \boldsymbol{\Gamma}_{0}\left(V_{\beta}^{\ell}(\Omega)\right) \quad \Longleftrightarrow \quad \psi_{0} \in H^{m-1 / 2}\left(\partial \Omega_{0}\right) \quad \text { and } \quad \psi^{ \pm} \in V_{\beta}^{m-1 / 2}\left(M^{ \pm}\right) .
$$

Although the norms (3.1) are well suited for the description of the asymptotic behavior of the solutions at the crack tips, the operators defined by the instantaneous Neumann elasticity operators (1.11) with domain $V_{\beta}^{\ell+1}(\Omega)^{2}$, are never of index zero, whatever the choice of the space weight index $\beta$, in contrast to the operator with domain $H^{1}(\Omega)^{2}$.

The reason for this is the presence of non-zero translations $a_{1} \mathbf{e}_{1}+a_{2} \mathbf{e}_{2}$ in the asymptotics of solutions at each crack tip as soon as the right hand side is more regular than the dual of the energy space $H^{1}(\Omega)^{2}$, together with the following two facts:

(i) if $\ell-\beta<0$, the weighted space $V_{\beta}^{\ell+1}(\Omega)^{2}$ is not contained in the energy space $H^{1}(\Omega)^{2}$;

(ii) if $\ell-\beta \geq 0$, non-zero translations $a_{1} \mathbf{e}_{1}+a_{2} \mathbf{e}_{2}$ do not belong to $V_{\beta}^{\ell+1}(\Omega)^{2}$.

Thus, translations are viewed as singularities of degree 0 by the weighted scale $V_{\beta}^{\ell+1}(\Omega)^{2}$. Constants and, more generally, smooth functions in the Cartesian variable $x$, are made admissible by a simple modification of the weighted norms (3.1), leading to the introduction of the so-called step-weighted spaces $[24,30]$ which in the case under consideration are closely connected with weighted Sobolev spaces [19].

They can be defined as follows: Let $m \in \mathbb{N}_{0}$ and $\beta \in \mathbb{R}$, with $-1<\beta \leq m$. For any integer $s, m-\beta-1<$ $s \leq m$, the space $V_{\beta}^{m+1, s}(\Omega)$ is the completion of $\mathfrak{C}^{\infty}(\Omega):=\mathfrak{C}_{0}^{\infty}(\Omega)+\mathcal{C}^{\infty}(\bar{\Omega})$ with respect to the norm

$$
\|w\|_{V_{\beta}^{m+1, s}(\Omega)}=\left(\sum_{j=s+1}^{m+1}\left\|\rho^{\beta-(m+1)+j} \nabla_{x}^{j} w\right\|_{L_{2}(\Omega)}^{2}+\sum_{j=0}^{s}\left\|\rho^{\beta-m+s} \nabla_{x}^{j} w\right\|_{L_{2}(\Omega)}^{2}\right)^{1 / 2} .
$$

We note that the condition $m-\beta-1<s$ ensures that any smooth function $w \in \mathcal{C}^{\infty}(\bar{\Omega})$ has a finite norm (3.5). If we choose $s=m$, we obtain the alternative class of weighted spaces $H_{\beta}^{m+1}(\Omega)=V_{\beta}^{m+1, m}(\Omega)$ defined as the functions with finite norm

$$
\|w\|_{H_{\beta}^{m+1}(\Omega)}=\left(\sum_{j=0}^{m+1}\left\|\rho^{\beta} \nabla_{x}^{j} w\right\|_{L_{2}(\Omega)}^{2}\right)^{1 / 2}
$$

where the weight is independent of the derivation order. The following result is a consequence of Hardy's inequality and is proved in [19] (see also [31], Th. 4.5.6 and Lem. 6.1.5).

\section{Lemma 3.1.}

(i) Let $m \in \mathbb{N}_{0}$ and $\beta \in \mathbb{R}$, with $-1<\beta \leq m$. We assume that $\beta$ is not an integer. For any integer $s$ with $m-\beta-1<s \leq m$, the space $H_{\beta}^{m+1}(\Omega)$ coincides with the space $V_{\beta}^{m+1, s}(\Omega)$.

(ii) For any $\beta>m$, the space $H_{\beta}^{m+1}(\Omega)$ coincides with the space $V_{\beta}^{m+1}(\Omega)$.

With the particular choice of $m-\beta \in(0,1)$ and $s=0$, the space $V_{\beta}^{m+1,0}(\Omega)$ is well defined (and coincides with $H_{\beta}^{m+1}(\Omega)$ ). We emphasize that, in comparison with the space $V_{\beta}^{m+1}(\Omega)$ that either includes, or excludes the 
constant 1 and the function $\ln r_{\mathfrak{i}}$ simultaneously, the step-weighted space $V_{\beta}^{m+1,0}(\Omega)$ with $m-\beta \in(0,1)$ includes the translation rigid motions, i.e. constants, and excludes the displacements resulting from the concentrated forces at the tips $\mathcal{O}^{i}$, i.e. logarithmic functions.

Let us also note that for a function $w \in V_{\beta}^{m+1,0}(\Omega)$ with $m \in \mathbb{N}_{0}$ and $m-\beta \in(0,1)$ the following relations are a consequence of a variant of Hardy's inequality, see [19], Lemma 1.2:

$$
\begin{gathered}
w(x)=\widetilde{w}(x)+\sum_{\mathfrak{i}=\mathbf{I}, \mathbf{I I}} \chi^{\mathfrak{i}}(x) b_{\mathbf{i}}, \quad \widetilde{w} \in V_{\beta}^{m+1}(\Omega), \quad b_{\mathfrak{i}} \in \mathbb{R}, \\
\|\widetilde{w}\|_{V_{\beta}^{m+1}(\Omega)}+\sum_{\mathbf{i}=\mathbf{I}, \mathbf{I I}}\left|b_{\mathfrak{i}}\right| \leq c\|w\|_{V_{\beta}^{m+1,0}(\Omega)}
\end{gathered}
$$

Here $\chi^{\mathfrak{i}} \in \mathcal{C}_{0}^{\infty}\left(\Omega_{0}\right)$ is a smooth cut-off function, which is equal to one in a neighbourhood of the point $\mathcal{O}^{\mathfrak{i}}$, with the property $\chi^{\mathbf{I}}(x) \chi^{\mathbf{I I}}(x)=0$ for $x \in \Omega$. Thus the supports of the cut-off functions are disjoint and the functions $\chi^{\mathbf{I}}\left(\right.$ respectively $\left.\chi^{\mathbf{I I}}\right)$ vanish in a vicinity of $\mathcal{O}^{\mathbf{I I}}$ (respectively $\mathcal{O}^{\mathbf{I}}$ ). We can choose $\chi^{\mathfrak{i}}$ such that $\operatorname{supp} \chi^{\mathfrak{i}} \subset \mathbb{B}^{\mathfrak{i}}:=\left\{x:\left|x-\mathcal{O}^{\mathfrak{i}}\right|<1\right\}$.

The sum in the left hand side of (3.8) is a norm in the space $V_{\beta}^{m+1,0}(\Omega)$ equivalent to the norm (3.5), which means that the space $V_{\beta}^{m+1,0}(\Omega)=H_{\beta}^{m+1}(\Omega)$, with $m-\beta \in(0,1)$ can be considered as a weighted space with separate asymptotics.

Let us point out that all the weighted spaces we have introduced are independent both algebraically and topologically on the specific choice of the functions $\rho$ and $\chi^{\mathfrak{i}}$.

We end this section by a statement which generalizes (3.7)-(3.8) and is proved in a similar way, see [19], Lemma 1.3. We use Cartesian coordinate systems $x_{\mathfrak{i}}=\left(x_{1} \mp 1, x_{2}\right)=:\left(x_{\mathfrak{i} 1}, x_{\mathfrak{i} 2}\right)$ with center $\mathcal{O}^{\mathfrak{i}}$.

Lemma 3.2. Let $m \in \mathbb{N}_{0}$ and $\beta \in \mathbb{R}$, with $-1<\beta<m$. We assume that $\beta$ is not an integer. Let $s$ be the integral part of $m-\beta$. Then any function $w \in V_{\beta}^{m+1, s}(\Omega)$ satisfies the following.

(i) There exist unique real numbers $b_{\mathbf{i}}^{\alpha},|\alpha| \leq s$, such that

$$
\begin{gathered}
w(x)=\widetilde{w}(x)+\sum_{\mathfrak{i}=\mathbf{I}, \mathbf{I I}} \chi^{\mathfrak{i}}(x) \sum_{|\alpha| \leq s} b_{\mathfrak{i}}^{\alpha} \frac{x_{\mathfrak{i}}^{\alpha}}{\alpha !}, \quad \widetilde{w} \in V_{\beta}^{m+1}(\Omega), \\
\|\widetilde{w}\|_{V_{\beta}^{m+1}(\Omega)}+\sum_{\mathfrak{i}=\mathbf{I}, \mathbf{I I}} \sum_{|\alpha| \leq s}\left|b_{\mathfrak{i}}^{\alpha}\right| \leq c\|w\|_{V_{\beta}^{m+1, s}(\Omega)} .
\end{gathered}
$$

Here, for $\alpha=\left(\alpha_{1}, \alpha_{2}\right), x_{\mathbf{i}}^{\alpha}$ denotes the monomial $x_{\mathfrak{i} 1}^{\alpha_{1}} x_{\mathfrak{i} 2}^{\alpha_{2}}$ and $\alpha !=\alpha_{1} ! \alpha_{2}$ !

(ii) The left hand side of (3.10) is a norm in the space $V_{\beta}^{m+1, s}(\Omega)$ equivalent to the norm (3.5).

(iii) The constants $b_{\mathbf{i}}^{\alpha}$ are the pointwise traces at the crack tip $\mathcal{O}^{\mathfrak{i}}$ of the derivatives of $u$ :

$$
b_{\mathfrak{i}}^{\alpha}=\partial^{\alpha} w\left(\mathcal{O}^{\mathfrak{i}}\right), \quad|\alpha| \leq s, \quad \mathfrak{i}=\mathbf{I}, \mathbf{I I} .
$$

(iv) The constants $b_{\mathbf{i}}^{\alpha}$ are all zero if and only if $w$ belongs to $V_{\beta}^{m+1}(\Omega)$.

(v) If, moreover, $s<m$ (i.e., $\beta>0$ ), the space $V_{\beta}^{m+1, s}(\Omega)$ is continuously imbedded in $\mathfrak{C}^{s}(\Omega)$, with $\mathfrak{C}^{s}(\Omega)$ the space of s-times continuously differentiable functions up to the boundary of $\Omega$.

\subsection{Basic regularity}

We will apply Theorem 2.1 again to problem $(1.2)-(1.4)$ in a new pair of spaces $\mathfrak{D}, \mathfrak{R}$ : the operator $\mathfrak{A}(t)$ is defined by the differential expression $\left\{L\left(x, t, \nabla_{x}\right), N\left(x, t, \nabla_{x}\right)\right\}$, the domain $\mathfrak{D}$ is taken as finite codimension subspace of $V_{\beta}^{\ell+1,0}(\Omega)^{2}$ and the target space as finite codimension subspace of $R_{\beta}^{\ell} V(\Omega)$ with

$$
R_{\beta}^{\ell} V(\Omega):=V_{\beta}^{\ell-1}(\Omega)^{2} \times V_{\beta}^{\ell-1 / 2}(\partial \Omega)^{2} .
$$


Here $\ell$ is the positive integer introduced in (1.6) and (1.7) and we assume $\ell-\beta>0$.

The following assertion can be proved either by combining the coercive weak formulation of problem (3.12) (cf. Th. 2.2) and the Kondrat'ev theory, or by calculations the dimensions of kernel and cokernel for the elasticity operator $: \mathfrak{D} \rightarrow \mathfrak{R}$ (see [31], Sect. 6.1 for details).

Theorem 3.3. Let the time $t$ be fixed. The instantaneous elasticity operator

$$
u \longmapsto\left\{L\left(x, t, \nabla_{x}\right), N\left(x, t, \nabla_{x}\right)\right\}(u) \quad \text { on } \Omega \times\left(\partial \Omega \backslash\left\{\mathcal{O}^{\mathbf{I}} \cup \mathcal{O}^{\mathbf{I I}}\right\}\right)
$$

defines an isomorphism $\mathfrak{A}(t): \mathfrak{D} \rightarrow \mathfrak{R}$ with

$$
\begin{gathered}
\mathfrak{D}=\left\{u \in V_{\beta}^{\ell+1,0}(\Omega)^{2}: \text { u satisfies orthogonality condition }(2.8)\right\}, \\
\mathfrak{R}=\left\{\{f, g\} \in R_{\beta}^{\ell} V(\Omega):\{f, g\} \text { satisfy compability condition }(2.7)\right\}
\end{gathered}
$$

if and only if $\ell-\beta \in\left(0, \frac{1}{2}\right)$.

Remark 3.4. By mere application of Lemma 3.2 about the splitting of $V_{\beta}^{\ell+1,0}(\Omega)$ we find that, if $\ell-\beta \in\left(0, \frac{1}{2}\right)$ the solution $u$ of the instantaneous elasticity operator with $\{f, g\} \in R_{\beta}^{\ell} V(\Omega)$ has an asymptotic expansion of the form

$$
u(x)=\widetilde{u}_{\beta}(x)+\sum_{\mathfrak{i}=\mathbf{I}, \mathbf{I I}} \chi^{\mathfrak{i}}(x)\left(a_{1, \mathbf{i}} \mathbf{e}_{1}+a_{2, \mathbf{i}} \mathbf{e}_{2}\right),
$$

where $\widetilde{u}_{\beta} \in V_{\beta}^{\ell+1}(\Omega)^{2}$ and $a_{1, \mathbf{i}}, a_{2, \mathbf{i}}$ are real constants. We recall that $\mathbf{e}_{1}$ and $\mathbf{e}_{2}$ are the unit vectors in $\mathbb{R}^{2}$.

Let us emphasize that the upper bound $\frac{1}{2}$ on the weight is due to the strongest singularities at the crack tips, which are associated with the exponent $\frac{1}{2}$, as already mentioned. Note that formula (3.14) will appear as a particular situation of a more general statement about the asymptotics of the solution of the instantaneous problem as $r_{\mathbf{i}} \rightarrow 0$, see (4.23).

Then we can apply the functional framework in Theorem 2.1 and prove:

Theorem 3.5. Let $l \in \mathbb{N}:=\{1,2, \ldots\}$ and $\beta>-1$ such that $\ell-\beta \in\left(0, \frac{1}{2}\right)$. Let the right hand side of the problem (1.2) verify condition (2.7) and the regularity assumption:

$$
\{f, g\} \in L_{\infty}\left(0, T ; R_{\beta}^{\ell} V(\Omega)\right) .
$$

Then there exists a unique solution $u \in L_{\infty}\left(0, T ; V_{\beta}^{\ell+1,0}(\Omega)\right)^{2}$ to problem (1.2)-(1.4) such that the orthogonality condition (2.8) is satisfied. The following estimate holds true,

$$
\|u ; t\|_{V_{\beta}^{\ell+1,0}(\Omega)} \leq c_{0} \mathrm{e}^{\delta_{0} t}\|\{f, g\} ; t\|_{R_{\beta}^{\ell} V(\Omega)},
$$

with positive constants $c_{0}$ and $\delta_{0}$ which are independent of $f, g$ and $t \in[0, T]$.

Definition (3.5) provides $V_{\beta}^{\ell+1,0}(\Omega) \subset H^{1}(\Omega)$ for $\ell-\beta \in(0,1)$. Therefore Theorem 3.5 gives, in a more precise way, the differentiability properties of the energy solution $(2.9)$. 
Proof of Theorem 3.5. Theorem 3.3 gives that the operators $\mathfrak{A}(t)$ defined by (3.12) are isomorphisms from $\mathfrak{D}$ onto $\mathfrak{R}$. Let

$$
\mathfrak{B}(t, \tau)=\left\{P\left(\cdot, t, \tau, \nabla_{x}\right), Q\left(\cdot, t, \tau, \nabla_{x}\right)\right\}
$$

be the Volterra operator kernel. Let us prove that $\mathfrak{B}(t, \tau)$ defines a family of continuous operators : $\mathfrak{D} \rightarrow \mathfrak{R}$.

The compatibility condition (2.7) was already shown in the proof of Theorem 2.2. Therefore it only remains to prove that there exists $c>0$ such that for any vector function $x \mapsto u(x)$ in $\mathfrak{D}$ and for a.e. $(t, \tau) \in \mathcal{T}(T)$

$$
\left\|\left\{P\left(\cdot, t, \tau, \nabla_{x}\right), Q\left(\cdot, t, \tau, \nabla_{x}\right)\right\} u\right\|_{R_{\beta}^{\ell} V(\Omega)} \leq c\|u\|_{V_{\beta}^{\ell+1,0}(\Omega)}
$$

We rely on the splitting (3.14) for the vector function $u=\left(u_{1}, u_{2}\right) \in V_{\beta}^{\ell+1,0}(\Omega)^{2}$. Obviously there holds

$$
\left\|\left\{P\left(\cdot, t, \tau, \nabla_{x}\right), Q\left(\cdot, t, \tau, \nabla_{x}\right)\right\} \widetilde{u}_{\beta}\right\|_{R_{\beta}^{\ell} V(\Omega)} \leq c\left\|\widetilde{u}_{\beta}\right\|_{V_{\beta}^{\ell+1}(\Omega)} .
$$

Moreover, thanks to the right factor $D\left(\nabla_{x}\right)$ in $\{P, Q\}$, the term $\left\{P\left(\cdot, t, \tau, \nabla_{x}\right), Q\left(\cdot, t, \tau, \nabla_{x}\right)\right\}$ is zero over constants (note that here, it would not be enough to invoke the orders of the operators because of the possible angular dependency of their coefficients). Therefore we have obtained (3.17). Thus Theorem 2.1 can be applied and yields the results of Theorem 3.5.

In the case of problem (1.13) the series (2.4) for the displacement field $u$ takes the form

$$
u(x, t)=\sum_{k=0}^{\infty} u^{k}(x, t)
$$

where $u^{k}$ is the solution to problem $\{L, N\} u^{k}=\left\{f^{k}, g^{k}\right\}$ with the orthogonality condition (2.8) and the right hand sides $\left\{f^{k}, g^{k}\right\}$ given as follows: $\left\{f^{0}, g^{0}\right\}=\{f, g\}$ and for $k \in \mathbb{N}$,

$$
\begin{aligned}
& \left\{f^{k}(x, t), g^{k}(x, t)\right\}=-\left\{D\left(-\nabla_{x}\right)^{\top} Y^{k}(x, t), D(n(x))^{\top} Y^{k}(x, t)\right\} \\
& \quad \text { with } Y^{k}(x, t)=\int_{0}^{t} B(x, t, \tau) D\left(\nabla_{x}\right) u^{k-1}(x, \tau) \mathrm{d} \tau
\end{aligned}
$$

Thus, the intermediate estimates (2.5) in the proof of Theorem 2.1 give for problem (1.13)

$$
\left\|u^{k} ; t\right\|_{V_{\beta}^{\ell+1,0}(\Omega)} \leq c_{0}\left(\delta_{0} t\right)^{k} \frac{1}{k !}\|\{f, g\} ; t\|_{R_{\beta}^{\ell} V(\Omega)} .
$$

\section{Singularities of instantaneous problems With Smooth Data}

For $\ell-\beta \in\left(0, \frac{1}{2}\right)$ as specified in Theorem 3.5, the elements $u$ of the space $V_{\beta}^{\ell+1,0}(\Omega)^{2}$ have pointwise traces at the crack tips $\mathcal{O}^{\mathbf{I}}$ and $\mathcal{O}^{\mathbf{I I}}$, but their associated stress fields $\sigma(u ; x)$ have not. The square root singularities of the instantaneous problems prevent any further use of Theorem 3.5 to improve the regularity result on the solution $u(x, t)$ of the creep problem. Since it is important to have a description of the non-continuous part of the stress field, we come back to the instantaneous problems

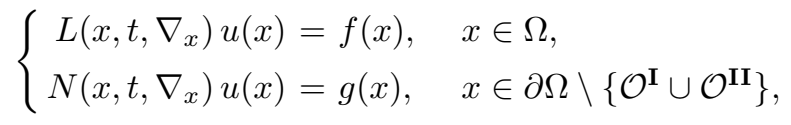

at fixed time $t$.

We consider this instantaneous problem (4.1) with $u \in H^{1}(\Omega)$ and the right hand side $\{f, g\} \in R_{\gamma}^{\ell} V(\Omega)$ for a weight index $\gamma$ such that $\ell-\gamma>\frac{1}{2}$. From Theorem 3.3 we know that $u$ belong to $V_{\beta}^{\ell+1}(\Omega)^{2}$ for all $\beta$ such 
that $\ell-\beta<\frac{1}{2}$, but the limit is sharp and $u$ does not belong to $V_{\gamma}^{\ell+1}(\Omega)^{2}$ in general. In this range of weights, the regularity result has to be replaced with a splitting result into regular and singular parts.

In this section we recall known techniques and results ending up at the description of asymptotics of $u$, in order to prepare for the next step, in which we have to take the Volterra kernel into account.

We start by the introduction of the Mellin operator pencils associated with the operators in (4.1) at the crack tips, and continue by recalling the Mellin transformation and its use to obtain splittings in regular and singular parts of solutions. Particular features of the pencils corresponding to crack problems for linear elasticity are then addressed. The splitting results are finally given first in the case of flat data, then for general smooth data.

\subsection{Mellin operator pencils}

The regularity properties (and the singularities) of solutions to problem (4.1) are determined by two model problems corresponding to each of the two crack tips $\mathcal{O}^{\mathfrak{i}}$ for $\mathfrak{i}=\mathbf{I}$, II. These problems are obtained by freezing the coefficients of $L$ and $N$ at $\mathcal{O}^{\mathfrak{i}}$, defining $L^{\mathfrak{i}}$ and $N^{\mathfrak{i} \pm}$ :

$$
\left\{\begin{aligned}
L^{\mathfrak{i}}\left(t, \nabla_{x}\right) & =D\left(-\nabla_{x}\right)^{\top} A\left(\mathcal{O}^{\mathfrak{i}}, t\right) D\left(\nabla_{x}\right) \\
N^{\mathfrak{i} \pm}\left(t, \nabla_{x}\right) & =D\left(\mp \mathbf{e}_{2}\right)^{\top} A\left(\mathcal{O}^{\mathfrak{i}}, t\right) D\left(\nabla_{x}\right) .
\end{aligned}\right.
$$

These problems are set on the plane with the semi-infinite crack

$$
\mathbb{K}:=\left\{x_{\mathfrak{i}} \in \mathbb{R}: r_{\mathfrak{i}}>0,\left|\varphi_{\mathfrak{i}}\right|<\pi\right\}
$$

and are written as :

$$
\left\{\begin{aligned}
L^{\mathfrak{i}}\left(t, \nabla_{x}\right) U\left(x_{\mathfrak{i}}\right) & =F\left(x_{\mathfrak{i}}\right), & & x_{\mathfrak{i}} \in \mathbb{K} \\
N^{\mathfrak{i} \pm}\left(t, \nabla_{x}\right) U\left(x_{\mathfrak{i}}\right) & =G^{ \pm}\left(x_{\mathfrak{i} 1}\right), & & \varphi_{\mathfrak{i}}= \pm \pi
\end{aligned}\right.
$$

The properties of problem (4.3) are related to the resolvent of the associated Mellin operator pencil $\mathcal{A}^{\mathfrak{i}}[t]$. In order to define this symbol, we first write the operators (4.2) in polar coordinates $(r, \varphi):=\left(r_{\mathfrak{i}}, \varphi_{\mathbf{i}}\right)$ :

$$
L^{\mathfrak{i}}\left(t, \nabla_{x}\right)=: r^{-2} \mathcal{L}^{\mathfrak{i}}\left(t, \varphi, \partial_{\varphi}, r \partial_{r}\right), \quad N^{\mathfrak{i} \pm}\left(t, \nabla_{x}\right)=: r^{-1} \mathcal{N}^{\mathfrak{i} \pm}\left(t, \partial_{\varphi}, r \partial_{r}\right) .
$$

Here the angular variable $\varphi$ belongs to the interval

$$
\Upsilon=(-\pi, \pi),
$$

$r$ to the half-axis $\mathbb{R}_{+}$and $\partial_{\varphi}=\partial / \partial \varphi, \partial_{r}=\partial / \partial r$. The Mellin operator pencil $\mathcal{A}^{\mathfrak{i}}[t]$ associated with problem (4.3) is the holomorphic operator valued function $\mathbb{C} \ni \lambda \mapsto \mathcal{A}^{\mathfrak{i}}[t](\lambda)$, where $\mathcal{A}^{\mathfrak{i}}[t](\lambda)$ is the operator acting in $\Upsilon$ according to

$$
\mathcal{A}^{\mathfrak{i}}[t](\lambda):=\left\{\begin{array}{ccc}
u & \longmapsto\left\{\mathcal{L}^{\mathfrak{i}}\left(t, \varphi, \partial_{\varphi}, \lambda\right), \mathcal{N}^{\mathfrak{i} \pm}\left(t, \partial_{\varphi}, \lambda\right)\right\}, \\
H^{\ell+1}(\Upsilon)^{2} & \longrightarrow & R^{\ell} H(\Upsilon) .
\end{array}\right.
$$

Here $R^{\ell} H(\Upsilon)$ is the target space:

$$
R^{\ell} H(\Upsilon):=H^{\ell-1}(\Upsilon)^{2} \times \mathbb{R}^{2} \times \mathbb{R}^{2} .
$$

We recall that the time $t \in[0, T]$ is fixed. Nevertheless, $t$ acts as a parameter and we will keep in our notations the dependency of the operators on $t$.

\subsection{Mellin transformation}

We fix one crack tip $\mathcal{O} \in\left\{\mathcal{O}^{\mathbf{I}}, \mathcal{O}^{\mathbf{I I}}\right\}$ and omit the index $\mathfrak{i} \in\{\mathbf{I}, \mathbf{I I}\}$ in the notation of coordinates, writing $r$ and $\varphi$ instead of $r_{\mathfrak{i}}$ and $\varphi_{\mathfrak{i}}$. After Kondrat'ev [15], the fundamental tool for the investigation of problem (4.3) is the Fourier-Laplace transform with respect to the variable $t=\ln r$, in other words the Mellin transform, which 
associates to a function $\mathbb{R}_{+} \times(-\pi, \pi) \ni(r, \theta) \mapsto U(r, \theta)$ with compact support in $r$ the function defined for all $\lambda \in \mathbb{C}$ by

$$
\mathcal{M}[U](\lambda, \theta)=\int_{0}^{+\infty} r^{-\lambda} U(r, \theta) \frac{\mathrm{d} r}{r} .
$$

The inverse Mellin transform $\mathcal{M}_{\xi}^{-1}$ can be expressed as (with $\xi \in \mathbb{R}$ )

$$
U(r, \theta)=\frac{1}{2 i \pi} \int_{\operatorname{Re} \lambda=\xi} r^{\lambda} \mathcal{M}[U](\lambda, \theta) \mathrm{d} \lambda .
$$

The Mellin transform can be naturally extended to functions belonging to the weighted spaces of the class $V$ : in the infinite sector $\mathbb{K}$ of opening $2 \pi$ we define the weighted spaces $V_{\beta}^{\ell}(\mathbb{K})$ like in (3.1) with the distance function $\rho$ replaced with the true distance $r$ to the vertex $\mathcal{O}$ of $\mathbb{K}$. The main result for the validity of the Mellin transform is

Lemma 4.1. For any $U \in V_{\beta}^{\ell+1}(\mathbb{K})$, the Mellin transform $\mathcal{M}[U]$ is well defined for any $\lambda$ with real part $\operatorname{Re} \lambda$ equal to $\ell-\beta$ and the inverse Mellin formula (4.5) holds for $\xi=\ell-\beta$.

We also define correspondingly the weighted trace spaces $V_{\beta}^{\ell-1 / 2}(\partial \mathbb{K})$, compare with (3.2), and the product space $R_{\beta}^{\ell} V(\mathbb{K})$ for right hand sides, see (3.11). For $\left\{F, G^{ \pm}\right\} \in R_{\beta}^{\ell} V(\mathbb{K})$ such that (4.9) holds, we have for any $\lambda$ with $\operatorname{Re} \lambda=\ell-\beta$ :

$$
\mathcal{A}^{\mathfrak{i}}[t](\lambda) \mathcal{M}[U](\lambda)=\left\{\mathcal{M}\left[r^{2} F\right], \mathcal{M}\left[r G^{ \pm}\right]\right\} .
$$

The above relation is the reason for the definition of $\mathcal{A}^{\mathfrak{i}}[t]$.

From the general theory of Agranovich and Vishik [1] we know that $\mathcal{A}^{\mathfrak{i}}[t](\lambda)$ is invertible for all $\lambda$ outside a discrete set $\Sigma^{\mathfrak{i}}$. Since the set $\Sigma^{\mathfrak{i}}$ coincides with the set of $\lambda$ such that the kernel of $\mathcal{A}^{\mathfrak{i}}[t]$ is not reduced to $\{0\}$, $\Sigma^{\mathfrak{i}}$ is called the spectrum of $\mathcal{A}^{\mathfrak{i}}[t]$ and for $\lambda \in \Sigma^{\mathfrak{i}}$ the non-zero solutions of

$$
\begin{cases}\mathcal{L}^{\mathfrak{i}}\left(t, \varphi, \partial_{\varphi}, \lambda\right) \mathcal{U}(\varphi)=0, & \varphi \in \Upsilon \\ \mathcal{N}^{\mathfrak{i} \pm}\left(t, \partial_{\varphi}, \lambda\right) \mathcal{U}(\varphi)=0, & \varphi= \pm \pi\end{cases}
$$

are the eigenvectors of $\mathcal{A}^{\mathfrak{i}}[t]$. Moreover, the power-law function of degree $\lambda \in \mathbb{C}$

$$
U(x, t)=r^{\lambda} \mathcal{U}(\varphi, t)
$$

is a solution to problem (4.3) with zero right hand side:

$$
\left\{\begin{array}{rlrl}
L^{\mathfrak{i}}\left(t, \nabla_{x}\right) U\left(x_{\mathfrak{i}}\right) & =0, & & x_{\mathfrak{i}} \in \mathbb{K}, \\
N^{\mathfrak{i} \pm}\left(t, \nabla_{x}\right) U\left(x_{\mathfrak{i}}\right) & =0, & \varphi_{\mathfrak{i}}= \pm \pi .
\end{array}\right.
$$

if and only if $\lambda$ belongs to $\Sigma^{\mathfrak{i}}$ and $\mathcal{U}$ is a corresponding eigenvector. Finally, $\lambda \mapsto \mathcal{A}^{\mathfrak{i}}[t](\lambda)^{-1}$ is meromorphic on the complex plane with poles in $\Sigma^{\mathfrak{i}}$.

The fundamental result concerning the solvability of the model problem (4.3) follows, $c f$. [15], Section 1:

Theorem 4.2. Let $\operatorname{Re} \Sigma^{\mathfrak{i}}$ be the set of real parts ${ }^{1}$ of the elements of $\Sigma^{\mathbf{i}}$.

(i) Let $\beta \in \mathbb{R}$, such that $\beta \notin \operatorname{Re} \Sigma^{\mathbf{i}}$. Then problem (4.3) realizes an isomorphism from $V_{\beta}^{\ell+1}(\mathbb{K})^{2}$ onto $R_{\beta}^{\ell} V(\mathbb{K})$. We have the representation formula for its solution:

$$
U=\frac{1}{2 i \pi} \int_{\operatorname{Re} \lambda=\ell-\beta} r^{\lambda} \mathcal{A}^{\mathfrak{i}}[t](\lambda)^{-1} \mathcal{H}(\lambda) \mathrm{d} \lambda, \quad \text { with } \quad \mathcal{H}=\left\{\mathcal{M}\left[r^{2} F\right], \mathcal{M}\left[r G^{ \pm}\right]\right\}
$$

\footnotetext{
${ }^{1}$ In the case of cracks $\operatorname{Re} \Sigma^{\mathbf{i}}$ coincides with the set of half-integers, see Section 4.3.
} 
and there holds the estimate

$$
\|U\|_{V_{\beta}^{\ell+1}(\mathbb{K})} \leq C_{\beta}\left\|\left\{F, G^{ \pm}\right\}\right\|_{R_{\beta}^{\ell} V(\mathbb{K})} .
$$

(ii) Let furthermore $\gamma<\beta$, such that $\gamma \notin \operatorname{Re} \Sigma^{i}$. For $\left\{F, G^{ \pm}\right\} \in R_{\beta}^{\ell} V(\mathbb{K}) \cap R_{\gamma}^{\ell} V(\mathbb{K})$, let $U_{\beta}$ and $U_{\gamma}$ be the solutions of problem (4.3) in $V_{\beta}^{\ell+1}(\mathbb{K})^{2}$ and $V_{\gamma}^{\ell+1}(\mathbb{K})^{2}$, respectively. There holds

$$
U_{\beta}=U_{\gamma}+\frac{1}{2 i \pi} \int_{\mathcal{C}} r^{\lambda} \mathcal{A}^{\mathfrak{i}}[t](\lambda)^{-1} \mathcal{H}(\lambda) \mathrm{d} \lambda
$$

Here $\mathcal{C}$ is a simple closed contour around all elements of $\Sigma^{\mathbf{i}}$ contained in the strip

$$
\{\lambda \in \mathbb{C}: \ell-\beta<\operatorname{Re} \lambda<\ell-\gamma\} .
$$

The key argument for the above results is the Mellin symbolic calculus: the solution $U_{\beta}$ is found via the formula

$$
U_{\beta}=\mathcal{M}_{\ell-\beta}^{-1}\left[\mathcal{A}^{\mathfrak{i}}[t](\lambda)^{-1}\left\{\mathcal{M}\left[r^{2} F\right], \mathcal{M}\left[r G^{ \pm}\right]\right\}\right]
$$

and the constant $C_{\beta}$ in (4.10) is any upper bound for $\mathcal{A}^{\mathfrak{i}}[t](\lambda)^{-1}$ in the norm of continuous operators $R^{\ell} H(\Upsilon) \rightarrow$ $H^{\ell+1}(\Upsilon)^{2}$ equipped with the parameter-dependent norms [1].

Moreover, $\mathcal{U}(\lambda):=\mathcal{A}^{\mathfrak{i}}[t](\lambda)^{-1} \mathcal{H}(\lambda)$ is a meromorphic extension of the Mellin transform of $U_{\beta}$ to the strip $\{\lambda \in \mathbb{C}: \ell-\beta<\operatorname{Re} \lambda<\ell-\gamma\}$. Note that the residue in (4.11) is the sum of the contributions $S\{\nu\}$ of each pole $\nu \in \Sigma$, with $\ell-\beta<\nu<\ell-\gamma$ : For $\varrho>0$ small enough (in the case of cracks any $\varrho<\frac{1}{2}$ is suitable)

$$
S\{\nu\}(x)=\frac{1}{2 i \pi} \int_{|\lambda-\nu|=\varrho} r^{\lambda} \mathcal{A}^{\mathfrak{i}}[t](\lambda)^{-1} \mathcal{H}(\lambda) \mathrm{d} \lambda .
$$

If $\nu$ is a non-zero element of $\Sigma$, it is a pole of order 1 and $S\{\nu\}$ has the form $r^{\nu} \mathcal{U}(\varphi)$ with an eigenvector $\mathcal{U}$ associated with the eigenvalue $\nu$.

\subsection{Spectrum of the operator pencils, eigenvalues $\frac{1}{2}$ and 1}

In our particular situation of a crack, we know much more about $\Sigma^{\mathfrak{i}}$ and the poles of $\mathcal{A}^{\mathfrak{i}}[t](\lambda)^{-1}$. Let us first recall that in [25], see also in [31], Section 7.4, and [27], it is proved that the spectrum of the associated operator pencil coincides with

$$
\mathbb{Z} \cup\left\{m+\frac{1}{2} \pm i \nu: m \in \mathbb{Z}\right\} \quad \nu \text { real independent of } m,
$$

in the general situation of the Dirichlet and Neumann problems for arbitrary selfadjoint systems which enjoy the polynomial property $[26,28]$. The fact that $\nu=0$ is well known for elasticity equations in isotropic and orthotropic materials and was established in [12] for homogeneous anisotropic elasticity. In [9] the equality $\nu=0$ is proved for arbitrary Douglis-Nirenberg systems with the same boundary operators on the two crack surfaces $\varphi= \pm \pi$. The situation $\nu \neq 0$ occurs $e . g$., with a crack inside the interface between two anisotropic bodies, we refer the reader to $[12,13,27]$ for related examples.

We gather in the following lemmas the facts which we will use in our analysis.

\section{Lemma 4.3.}

(i) The spectrum $\Sigma^{\mathfrak{i}}$ contains integers and semi-integers, i.e., $\Sigma^{\mathfrak{i}}=\frac{1}{2} \mathbb{Z}$.

(ii) All non-zero eigenvalues $\lambda \in \frac{1}{2} \mathbb{Z}$ are algebraically simple, their geometric multiplicities are equal to 2 . The full algebraic multiplicity of the eigenvalue $\lambda=0$ is 4 , its partial algebraic multiplicities are equal to 2 . 
(iii) Eigenfunctions corresponding to positive integers $\lambda \in \mathbb{N}$ are traces on $\Upsilon$ of vector polynomials of degree $\lambda$, solutions of problem (4.9). In particular, for each $\lambda \in \mathbb{N}$ there are exactly two linear independent polynomials.

For the specific problems considered in the paper, we are interested only in the eigenvalues $\lambda=\frac{1}{2}$ and $\lambda=1$, and in the corresponding power solutions (4.8) of problem (4.9).

The solvability of $\mathcal{A}^{\mathfrak{i}}[t](\lambda)$ when $\lambda$ belongs to its spectrum is related to dual eigenfunctions corresponding to the eigenvalue $-\lambda$ via the following duality product between functions $\mathcal{U}$ and $\mathcal{U}^{*} \in H^{1}(\Upsilon)$ :

$$
\mathscr{X}\left(\mathcal{U} \mid \mathcal{U}^{*}\right)=\left.\int_{-\pi}^{\pi} \mathcal{U}^{*}(\varphi)^{\top} \frac{\mathrm{d}}{\mathrm{d} \lambda} \mathcal{L}^{\mathfrak{i}}\left(t, \varphi, \partial_{\varphi}, \lambda\right)\right|_{\lambda=\frac{1}{2}} \mathcal{U}(\varphi) \mathrm{d} \varphi+\left.\sum_{ \pm} \mathcal{U}^{*}( \pm \pi)^{\top} \frac{\mathrm{d}}{\mathrm{d} \lambda} \mathcal{N}^{\mathfrak{i} \pm}\left(t, \partial_{\varphi}, \lambda\right)\right|_{\lambda=\frac{1}{2}} \mathcal{U}( \pm \pi)
$$

Here follow properties concerning the eigenvalue $\lambda=\frac{1}{2}$.

Lemma 4.4. We recall that $\Upsilon$ is the interval $(-\pi, \pi)$.

(i) The power solutions (4.8) of problem (4.9) with $\lambda=\frac{1}{2}$ have a basis of the form $\left\{V_{1, \mathfrak{i}}(x, t), V_{2, \mathbf{i}}(x, t)\right\}$ with

$$
V_{1, \mathfrak{i}}(x, t)=r^{1 / 2} \mathcal{V}_{1, \mathfrak{i}}(\varphi, t) \quad \text { and } \quad V_{2, \mathfrak{i}}(x, t)=r^{1 / 2} \mathcal{V}_{2, \mathfrak{i}}(\varphi, t)
$$

Thanks to $(1.6)$ we can choose $\mathcal{V}_{n, \mathfrak{i}} \in L_{\infty}\left(0, T ; C^{\infty}(\bar{\Upsilon})\right)^{2}, n=1,2$.

(ii) The eigenfunctions $\left(\mathcal{V}_{j, \mathbf{i}}^{*}\right)_{j=1,2}$ corresponding to the eigenvalue $-\frac{1}{2}$ of problem (4.7) can be selected so that the following biorthogonality condition is satisfied

$$
\mathscr{X}\left(\mathcal{V}_{n, \mathfrak{i}}(\cdot, t) \mid \mathcal{V}_{j, \mathfrak{i}}^{*}(\cdot, t)\right)=\delta_{n, j}, \quad n, j=1,2
$$

where $\delta_{n, j}$ is the Kronecker symbol, and as a consequence of the boundedness of $\mathcal{V}_{n, \mathbf{i}}$ in $t$, one gets $\mathcal{V}_{j, \mathfrak{i}}^{*} \in L_{\infty}\left(0, T ; C^{\infty}(\bar{\Upsilon})\right)^{2}$

(iii) The boundary value problem

$$
\mathcal{L}^{\mathfrak{i}}\left(t, \varphi, \partial_{\varphi}, \frac{1}{2}\right) \mathcal{U}(\varphi)=\mathcal{F}(\varphi), \quad \varphi \in \Upsilon ; \quad \mathcal{N}^{\mathfrak{i} \pm}\left(t, \partial_{\varphi}, \frac{1}{2}\right) \mathcal{U}( \pm \pi)=\mathcal{G}^{ \pm}
$$

with the right hand sides $\left\{\mathcal{F}, \mathcal{G}^{ \pm}\right\} \in R^{\ell} H(\Upsilon)$ admits a solution $\mathcal{U} \in H^{\ell+1}(\Upsilon)^{2}$ if and only if the following compatibility conditions are verified

$$
\int_{-\pi}^{\pi} \mathcal{V}_{j, \mathbf{i}}^{*}(\varphi, t)^{\top} \mathcal{F}(\varphi) \mathrm{d} \varphi+\sum_{ \pm} \mathcal{V}_{j, \mathbf{i}}^{*}( \pm \pi, t)^{\top} \mathcal{G}^{ \pm}=0, \quad j=1,2
$$

Any solution is determined up to linear combinations $c_{1} \mathcal{V}_{1, \mathfrak{i}}+c_{2} \mathcal{V}_{2, \mathbf{i}}$. However by imposing the orthogonality conditions

$$
\mathscr{X}\left(\mathcal{U} \mid \mathcal{V}_{j, \mathbf{i}}^{*}\right)=0, \quad j=1,2
$$

a unique solution is obtained. Furthermore, the following estimate holds true:

$$
\|\mathcal{U}\|_{H^{\ell+1}(\Upsilon)} \leq C\left\|\left\{\mathcal{F}, \mathcal{G}^{ \pm}\right\}\right\|_{R^{\ell} H(\Upsilon)}
$$


Proof. The part (ii) is a particular case of general normalization and biorthogonality conditions presented in [20], as it is given in ([31], p. 65). In [27] is provided the mechanical interpretation of the conditions, as well as the methods of selection of the bases $\left\{\mathcal{V}_{1, \mathfrak{i}}, \mathcal{V}_{2, \mathbf{i}}\right\}$, adapted to different fracture criteria. The part (iii) is the standard Fredholm alternative from the theory of ordinary differential equations.

Remark 4.5. For isotropic materials, standard normalization conditions for the eigenfunctions $\mathcal{V}_{1, \mathfrak{i}}$ and $\mathcal{V}_{2, \mathbf{i}}$ deal with the associated stresses on the polar axis $\{x: r>0, \varphi=0\}$ prolongating the crack. As shown in [27], these normalization conditions

$$
\left\{\begin{array}{l}
\left(D\left(\mathbf{e}_{2}\right) \mathbf{e}_{2}\right)^{\top} A(\mathcal{O}, t) D\left(\nabla_{x}\right) V_{n, \mathbf{i}}\left(x_{1}, 0\right)=(2 \pi r)^{-1 / 2} \delta_{n, 1} \text { (normal stress) } \\
\left(D\left(\mathbf{e}_{2}\right) \mathbf{e}_{1}\right)^{\top} A(\mathcal{O}, t) D\left(\nabla_{x}\right) V_{n, \mathbf{i}}\left(x_{1}, 0\right)=(2 \pi r)^{-1 / 2} \delta_{n, 2} \text { (shear stress) }
\end{array}\right.
$$

can be satisfied for anisotropic materials as well.

Besides its first assertion which is derived with the help of simple algebraic calculations, the next lemma delivers the same facts about eigenvalues \pm 1 as Lemma 4.4 about $\pm \frac{1}{2}$.

\section{Lemma 4.6.}

(i) $A$ basis $\left\{W_{1, \mathfrak{i}}(x, t)=r \mathcal{W}_{1, \mathfrak{i}}(\varphi, t), W_{2, \mathfrak{i}}(x)=r \mathcal{W}_{2, \mathfrak{i}}(\varphi)\right\}$ of the power solutions (4.8) of problem (4.9) with $\lambda=1$ is given as follows:

$$
W_{1, \mathfrak{i}}(x, t)=x_{1} \mathbf{e}_{1}-x_{2} \mathbf{A}^{\mathfrak{i}}(t), \quad W_{2, \mathfrak{i}}(x)=x_{1} \mathbf{e}_{2}-x_{2} \mathbf{e}_{1},
$$

where $\mathbf{A}^{\mathfrak{i}}(t)=\mathcal{E}_{+} A^{\prime \prime}\left(\mathcal{O}^{\mathfrak{i}}, t\right)^{-1} A^{\prime}\left(\mathcal{O}^{\mathfrak{i}}, t\right) \in L_{\infty}(0, T)^{2}$,

$$
\mathcal{E}_{ \pm}=\left(\begin{array}{cc}
0 & 2^{ \pm 1 / 2} \\
1 & 0
\end{array}\right), \quad A^{\prime}=\left(\begin{array}{l}
A_{21} \\
A_{31}
\end{array}\right), \quad A^{\prime \prime}=\left(\begin{array}{ll}
A_{22} & A_{23} \\
A_{32} & A_{33}
\end{array}\right)
$$

i.e., the matrix $\left(A^{\prime}, A^{\prime \prime}\right)$ is the lower $2 \times 3$-submatrix of the matrix $A$.

(ii) There exist eigenfunctions $\mathcal{W}_{1}^{*}$ and $\mathcal{W}_{2}^{*}$ associated with the eigenvalue -1 of problem (4.7) so that the properties corresponding to (ii) and (iii) of Lemma 4.4 hold for $\lambda=1$.

Let us point out that the first solution in (4.21) corresponds to the loading along the crack i.e., $\sigma\left(W_{1, \mathfrak{i}} ; x, t\right)=$ $(c(t), 0,0)^{\top}$. The second solution in $(4.21)$ is a rotation about the point $\mathcal{O}$. Note that the two other rigid motions, the shifts along the axes $x_{1}$ and $x_{2}$, are present in (3.14) as detached terms of the asymptotics.

\subsection{Splitting of solutions for regular data with zero values at crack tips}

We investigate the solutions $u$ provided by Theorem 3.3 when the data are more regular, but still flat, which means that their traces at the crack tips, if they exist, are zero. The extension to more realistic regular data is discussed in the next Section 4.5.

Theorem 4.7. Let $\gamma$ be such that

$$
\gamma>-1 \quad \text { and } \quad \ell-\gamma \in\left(1, \frac{3}{2}\right)
$$

Let $\{f, g\} \in R_{\gamma}^{\ell} V(\Omega)$ satisfying the compatibility condition (2.7) and $u$ be the energy solution of the instantaneous problem (4.1) satisfying the orthogonality condition (2.8). We know, Theorem 3.3, that u belongs to $V_{\beta}^{\ell+1,0}(\Omega)^{2}$ for all $\beta$ such that $\ell-\beta \in\left(0, \frac{1}{2}\right)$, with the asymptotics (3.14). There holds moreover

$$
u(x)=\widetilde{u}_{\gamma}(x)+\sum_{\mathfrak{i}=\mathbf{I}, \mathbf{I I}} \chi^{\mathfrak{i}}(x) \sum_{n=1}^{2}\left\{a_{n, \mathfrak{i}} \mathbf{e}_{n}+b_{n, \mathfrak{i}} V_{n, \mathfrak{i}}\left(x_{\mathfrak{i}}, t\right)+c_{n, \mathfrak{i}} W_{n, \mathfrak{i}}\left(x_{\mathfrak{i}}, t\right)\right\}
$$


where $\widetilde{u}_{\gamma} \in V_{\gamma}^{\ell+1}(\Omega)^{2}$ and $a_{n, \mathbf{i}}, b_{n, \mathbf{i}}, c_{n, \mathbf{i}}$ are real numbers. We recall that $x_{\mathbf{I}}=\left(x_{1}-1, x_{2}\right)$ and $x_{\mathbf{I I}}=\left(x_{1}+1, x_{2}\right)$. The displacements

$$
V_{n, \mathbf{i}}\left(x_{\mathfrak{i}}, t\right)=r_{\mathfrak{i}}^{1 / 2} \mathcal{V}_{n, \mathbf{i}}\left(\varphi_{\mathbf{i}}, t\right) \quad \text { and } \quad W_{n, \mathbf{i}}\left(x_{\mathfrak{i}}, t\right)=r_{\mathfrak{i}} \mathcal{W}_{n, \mathbf{i}}\left(\varphi_{\mathbf{i}}, t\right)
$$

are those introduced in Lemmas 4.4 (i) and 4.6 (i), respectively, for each crack tip.

Proof. We choose $\beta$ such that $\ell-\beta+1=\ell-\gamma$. By Theorem 3.3, we know that $u$ has the expansion (3.14). Since the coefficients of the operators $L$ and $N$ are smooth, we can immediately deduce that

$$
\left\{F_{\mathfrak{i}}, G_{\mathfrak{i}}^{ \pm}\right\}:=\left\{L^{\mathfrak{i}}, N^{\mathfrak{i} \pm}\right\}\left(\chi^{\mathfrak{i}} \widetilde{u}_{\beta}\right) \in R_{\gamma}^{\ell} V(\mathbb{K}), \quad \mathfrak{i}=\mathbf{I}, \mathbf{I I}
$$

where $\chi^{\mathfrak{i}} \widetilde{u}_{\beta}$ is defined on the entire sector $\mathbb{K}$ by extension by 0 . Then we apply Theorem 4.2 to the data $\left\{F_{\mathfrak{i}}, G_{\mathfrak{i}}^{ \pm}\right\}$, which also belong to $R_{\beta}^{\ell} V(\mathbb{K})$ since they have compact support. The solution $U_{\beta}=U_{\beta, \mathbf{i}}$ coincides with $\chi^{\mathfrak{i}} \widetilde{u}_{\beta}$. The regular part is $U_{\gamma}=U_{\gamma, \mathfrak{i}}$. The residue formula for $\mathfrak{i}=\mathbf{I}, \mathbf{I I}$, combined with Lemmas 4.4 and 4.6 yields that the asymptotic parts appear for $\nu=\frac{1}{2}$ and 1 and that they have the form in (4.23). Setting

$$
\widetilde{u}_{\gamma}(x)=\sum_{\mathfrak{i}=\mathbf{I}, \mathbf{I I}} \chi^{\mathfrak{i}}(x) U_{\gamma, \mathbf{i}}\left(x_{\mathfrak{i}}\right)
$$

we end the proof of the theorem.

\section{Remark 4.8.}

(i) Since the functions $W_{n, \mathbf{i}}$ are polynomial, the expansion (4.23) can be reformulated as

$$
u(x)=\widetilde{w}_{\gamma}(x)+\sum_{\mathfrak{i}=\mathbf{I}, \mathbf{I I}} \chi^{\mathfrak{i}}(x) \sum_{n=1}^{2} b_{n, \mathbf{i}} V_{n, \mathbf{i}}\left(x_{\mathfrak{i}}, t\right) \quad \text { with } \quad \widetilde{w}_{\gamma} \in V_{\gamma}^{\ell+1,1}(\Omega)^{2} .
$$

Here the $b_{n, \boldsymbol{i}}$ are the same constants as in (4.23). Note that with $\gamma$ in the range (4.22), for any displacement $w \in V_{\gamma}^{\ell+1,1}(\Omega)^{2}$ the stresses have pointwise values at the crack tips $\mathcal{O}^{\mathbf{i}}$, cf. Lemma 3.2. In particular, the associated normal stresses $g^{ \pm}=N\left(x, t, \nabla_{x}\right) \widetilde{w}_{\gamma}$ on $M^{ \pm}$have pointwise values $g^{ \pm}\left(\mathcal{O}^{\mathfrak{i}}\right)$ at the crack tips.

(ii) In principle we can choose $\gamma$ and $l$ so that $\ell-\gamma$ is still larger than prescribed by (4.22). But the latter limitation suffices to obtain a description of the stresses modulo continuous fields (i.e. the displacements modulo $\mathcal{C}^{1}$ fields). Moreover taking variable coefficients into account is easier with (4.22) (no shadows are present).

\subsection{Smooth data for tractions. Values at crack tips}

Until now we have considered right hand sides

$$
\{f, g\} \in R_{\gamma}^{\ell} V(\Omega) \quad \text { with } \quad \ell-\gamma \in\left(1, \frac{3}{2}\right) .
$$

For the volume forces $f$, since $V_{\underline{\gamma}}^{\ell-1}(\Omega)=H_{\gamma}^{\ell-1}(\Omega)$, we have no other choice than $f \in V_{\gamma}^{\ell-1}(\Omega)^{2}$; moreover, any function, sufficiently smooth in $\bar{\Omega}$, belongs to this space.

In contrast, for the tractions $g$ we note that the weight $\rho^{\gamma-l+1}$ is unbounded and forces the elements of $V_{\gamma}^{\ell+1}(\Omega)$ to have pointwise value zero in $\mathcal{O}^{i}$ for their gradients. Whereas for the regular part $\widetilde{w}_{\gamma}$ in $(4.24)$, the pointwise values $g^{ \pm}\left(\mathcal{O}^{\mathbf{i}}\right)$ are zero by virtue of assumption (4.25), for a general displacement $w \in V_{\gamma}^{\ell+1,1}(\Omega)^{2}$, these values are not zero. However they cannot be any combination of real values: since

$$
\left.N\left(x, t, \nabla_{x}\right)\right|_{M^{ \pm}}=D\left(\mp \mathbf{e}_{2}\right)^{\top} A\left(x_{1}, 0, t\right) D\left(\nabla_{x}\right)
$$


we obtain the compatibility conditions for the normal stresses of a regular displacement:

$$
g^{-}\left(\mathcal{O}^{\mathfrak{i}}\right)=-g^{+}\left(\mathcal{O}^{\mathfrak{i}}\right), \quad \mathfrak{i}=\mathbf{I}, \mathbf{I I}
$$

We cannot be satisfied with the assumption $g^{ \pm}\left(\mathcal{O}^{\mathfrak{i}}\right)=0$ because

(i) Such a property is not acceptable in many physically justified examples, in particular in the case of the uniform pressure from the interior of the crack (oil stocks).

(ii) We have to keep in mind the creep problem where a recursive procedure has to be applied, see (3.19). Since only one out of the two polynomial displacements $\mathcal{W}_{j}$ is a rigid motion, the iterative procedure does not conserve zero pointwise values for the normal stresses.

Now is it justified to impose the compatibility condition (4.27)? The answer may be yes only if the Volterra kernel $B$ has differentiable coefficients with respect to $x$. In the case of sectorial coefficients, condition (4.27) cannot be kept along the iterative procedure.

In order to provide precise definitions of the spaces for the boundary data $g$, it is necessary to examine more carefully spaces of traces. We have already introduced in (3.3) the space $V_{\beta}^{\ell-1 / 2}(\partial \Omega)$. It coincides with the trace space of $V_{\beta}^{\ell}(\Omega)$, see (3.4). Considering now the space $V_{\gamma}^{\ell, 0}(\Omega)=H_{\gamma}^{\ell}(\Omega)$ with $\gamma$ and $l$ as in (4.22), we find that a relation of type (3.4) is no longer true. Indeed, on the model of (3.3) we define the space $H_{\gamma}^{\ell-1 / 2}(\partial \Omega)$ as the direct sum

$$
H_{\gamma}^{\ell-1 / 2}(\partial \Omega):=H^{\ell-1 / 2}\left(\partial \Omega_{0}\right) \oplus H_{\gamma}^{\ell-1 / 2}\left(M^{+}\right) \oplus H_{\gamma}^{\ell-1 / 2}\left(M^{-}\right)
$$

where $H_{\gamma}^{\ell-1 / 2}\left(M^{ \pm}\right)$is the space with the norm, $c f .(3.2)$ :

$$
\|\psi\|_{H_{\gamma}^{\ell-1 / 2}\left(M^{ \pm}\right)}=\left\{\sum_{k=0}^{\ell-1}\left\|\rho^{\gamma} \partial_{1}^{k} \psi\right\|_{L_{2}\left(M^{ \pm}\right)}^{2}+I_{l, \gamma}^{ \pm}(\psi)\right\}^{1 / 2},
$$

where the seminorms $I_{l, \gamma}^{ \pm}(\psi)$ are defined in (3.2). Each space $H_{\gamma}^{\ell-1 / 2}\left(M^{ \pm}\right)$is the trace space $\boldsymbol{\Gamma}_{0}^{ \pm}\left(H_{\gamma}^{\ell}(\Omega)\right)$ separately, and consists of functions $\psi^{ \pm}$admitting the representation

$$
\psi^{ \pm}\left(x_{1}\right)=\widetilde{\psi}^{ \pm}\left(x_{1}\right)+\sum_{\mathbf{i}=\mathbf{I}, \mathbf{I I}} \chi^{\mathfrak{i}}\left(x_{1}, 0\right) \psi^{ \pm}\left(\mathcal{O}^{\mathfrak{i}}\right) \quad \text { with } \quad \widetilde{\psi}^{ \pm} \in V_{\gamma}^{\ell-1 / 2}\left(M^{ \pm}\right) .
$$

In the space (4.28), the elements $\widetilde{\psi}^{ \pm}$are independent from each other, and $\psi^{ \pm}\left(\mathcal{O}^{\mathfrak{i}}\right)$ can be chosen as any constants. On the other hand, formula (3.7) gives the same representation (4.29) for the trace $\psi \in \boldsymbol{\Gamma}_{0}\left(H_{\gamma}^{\ell}(\Omega)\right.$ ) but with the relationship

$$
\psi^{+}\left(\mathcal{O}^{\mathfrak{i}}\right)=\psi^{-}\left(\mathcal{O}^{\mathfrak{i}}\right), \quad \mathfrak{i}=\mathbf{I}, \mathbf{I I},
$$

since the constants $b^{\mathfrak{i}}$ are common in (3.7) for both surfaces of the crack $M$.

Note that (4.30) differs from (4.27) and the change of signs in (4.27) results from the matrix $D\left(\mp \mathbf{e}_{2}\right)$ in the Neumann elasticity operator (4.27). To provide the correct relationship between pointwise values at crack tips of applied external surface loading, we introduce the trace operator $\boldsymbol{\Gamma}_{1}=\partial_{n}$ for the derivative along the outward normal. For the same range of weight indices $\gamma$, due to the opposite direction of the normal on $M^{ \pm}$, we obtain

$$
\begin{aligned}
\psi \in \boldsymbol{\Gamma}_{1}\left(H_{\gamma}^{\ell+1}(\Omega)\right) \Longleftrightarrow & \psi_{0} \in H^{\ell-1 / 2}\left(\Gamma_{0}\right) \text { and } \\
& \psi^{ \pm} \in H_{\gamma}^{\ell-1 / 2}\left(M^{ \pm}\right) \text {with } \psi^{+}\left(\mathcal{O}^{\mathfrak{i}}\right)=-\psi^{-}\left(\mathcal{O}^{\mathfrak{i}}\right), \mathfrak{i}=\mathbf{I}, \mathbf{I I} .
\end{aligned}
$$


The following space for data $\{f, g\}$ is now justified mechanically:

$$
R_{\gamma}^{\ell, 1} V(\Omega):=V_{\gamma}^{\ell-1}(\Omega)^{2} \times \boldsymbol{\Gamma}_{1}\left(V_{\gamma}^{\ell+1,1}(\Omega)\right)^{2} .
$$

We point out, that in the right hand side of (4.32) the symbol $V$ can be replaced by $H$ since $H_{\gamma}^{\ell-1}(\Omega)=V_{\gamma}^{\ell-1}(\Omega)$ and $H_{\gamma}^{\ell+1}(\Omega)=V_{\gamma}^{\ell+1,1}(\Omega)$ for $\gamma, l$ satisfying (4.22) (Lemma 3.1). We use the symbol $V$ in (4.32) for the only reason to distinguish the space $R_{\gamma}^{\ell, 1} V(\Omega)$ from the maximal space for datum $\{f, g\}$ defined as

$$
R_{\gamma}^{\ell} H(\Omega):=H_{\gamma}^{\ell-1}(\Omega)^{2} \times H_{\gamma}^{\ell-1 / 2}(\partial \Omega)^{2}
$$

In view of (4.28), space (4.33) is intrinsic for problem (4.1) mathematically.

\subsection{Splitting of solutions with general smooth data}

The model problem (4.3) with right-hand sides satisfying the compatibility condition (4.31) admits a smooth particular solution: explicit calculations give

Lemma 4.9. Particular solutions of problem (4.3) with the right hand sides $F=0$ and $G^{ \pm}$of the form $G^{ \pm}\left(x_{1}\right)= \pm G$ with $G \in \mathbb{R}^{2}$ are given by

$$
W_{0, \mathfrak{i}}(x, t)=-x_{2} G_{\mathfrak{i}}(t), \quad \text { with } \quad G_{\mathfrak{i}}(t)=\mathcal{E}_{+} A^{\prime \prime}\left(\mathcal{O}^{\mathfrak{i}}, t\right)^{-1} \mathcal{E}_{-} G \in \mathbb{R}^{2}
$$

with $\mathcal{E}_{ \pm}$and $A^{\prime \prime}$ defined in Lemma 4.6.

We immediately deduce from this lemma the following extension of Theorem 4.7.

Theorem 4.10. Let $\gamma$ be such that $\ell-\gamma \in\left(1, \frac{3}{2}\right)$. Let $\{f, g\} \in R_{\gamma}^{\ell, 1} V(\Omega)$, cf. (4.32), satisfying the compatibility condition (2.7) and let $u$ be the energy solution of the instantaneous problem (4.1) satisfying the orthogonality condition (2.8). There holds

$$
u(x)=\widetilde{w}_{\gamma}(x)+\sum_{\mathfrak{i}=\mathbf{I}, \mathbf{I I}} \chi^{\mathfrak{i}}(x) \sum_{n=1}^{2} b_{n, \mathbf{i}} V_{n, \mathfrak{i}}\left(x_{\mathfrak{i}}, t\right) \quad \text { with } \quad \widetilde{w}_{\gamma} \in V_{\gamma}^{\ell+1,1}(\Omega)^{2}
$$

The full decomposition of $u$ can be written as

$$
u(x)=\widetilde{u}_{\gamma}(x)+\sum_{\mathfrak{i}=\mathbf{I}, \mathbf{I I}} \chi^{\mathfrak{i}}(x)\left[\sum_{n=1}^{2}\left(a_{n, \mathfrak{i}} \mathbf{e}_{n}+b_{n, \mathfrak{i}} V_{n, \mathfrak{i}}\left(x_{\mathfrak{i}}, t\right)+c_{n, \mathfrak{i}} W_{n, \mathfrak{i}}\left(x_{\mathfrak{i}}, t\right)\right)-x_{2} G_{\mathfrak{i}}\right]
$$

with $\widetilde{u}_{\gamma} \in V_{\gamma}^{\ell+1}(\Omega)^{2}$, real constants $a_{n, \mathfrak{i}}, b_{n, \mathfrak{i}}, c_{n, \mathfrak{i}}$, and $G_{\mathfrak{i}}$ according to Lemma 4.9 .

Remark 4.11. If, instead of a right hand side in $\{f, g\} \in R_{\gamma}^{\ell, 1} V(\Omega)$, we take a right hand side $\{f, g\} \in R_{\gamma}^{\ell} H(\Omega)$ (i.e., without compatibility conditions for tractions at crack tips) we find, instead of (4.35), a splitting of the form

$$
u(x)=\widetilde{w}_{\gamma}(x)+\sum_{\mathfrak{i}=\mathbf{I}, \mathbf{I I}} \chi^{\mathfrak{i}}(x) \sum_{n=1}^{2}\left(b_{n, \mathbf{i}} V_{n, \mathfrak{i}}\left(x_{\mathfrak{i}}, t\right)+d_{n, \mathfrak{i}} X_{n, \mathfrak{i}}\left(x_{\mathfrak{i}}, t\right)\right)
$$

with the regular part $\widetilde{w}_{\gamma} \in V_{\gamma}^{\ell+1,1}(\Omega)^{2}$. Here $X_{n, \mathbf{i}}$, for $n=1,2$, are logarithmic singularities, of the form $r_{\mathfrak{i}}\left(\ln r_{\mathfrak{i}} \mathcal{W}^{1}\left(\varphi_{\mathfrak{i}}, t\right)+\mathcal{W}^{0}\left(\varphi_{\mathfrak{i}}, t\right)\right)$ and $\left(d_{n, \mathfrak{i}}\right)_{n=1,2}$ are the two components of $g^{+}\left(\mathcal{O}^{\mathfrak{i}}\right)+g^{-}\left(\mathcal{O}^{\mathfrak{i}}\right)$. 


\section{Power AND POWER-LOGARITHMiC SOlutions of InStantaneous PROBlems WITH SINGULAR DATA}

In this section, we develop new preparatory material in view of the proof of our results relating to the creep problem (1.2)-(1.4). The idea we have in mind is to make use of the representation of solutions of the creep problem as a series (3.18) where the terms $u_{k}$ of the series solve $\{L, N\} u_{k}=\left\{f_{k}, g_{k}\right\}$ with $\left\{f_{k}, g_{k}\right\}$ defined by (3.19). We start the analysis by applying Theorem 4.10 to the first term $u_{0}$. Then, we obtain for $\left\{f_{1}, g_{1}\right\}$ a right hand side which contains itself a power singularity. This singular right hand side gives rise, in general, to a power-logarithmic singularity for $u_{1}$, because of a resonance between the data and the inverse of the Mellin operator pencil at $\lambda_{0}=\frac{1}{2}$.

The investigation of singular data in that sense is the purpose of this section. Relying on Kondrat'ev's theory, reformulated with the help of the Mellin transform (see Sect. 4.2), we prove sharp estimates about power-logarithmic singularities, independently of their degree. This will lead to our results regarding the general structure of singularities of the creep problem ("logarithmic packets"). Finally, to prepare for the situations where logarithmic terms do not propagate (or are absent), we investigate in more detail the angular structure of singularities, using the Cayley transform as initiated in [8].

\subsection{Volterra kernel in polar coordinates}

Recall that $\mathfrak{i}=\mathbf{I}, \mathbf{I I}$ indicates the crack tip $\mathcal{O}^{\mathfrak{i}}$ while \pm corresponds to the crack's surfaces $M^{ \pm}$. Freezing coefficients of differential operators associated to the relaxation kernel,

$$
\left\{\begin{array}{l}
P\left(x, t, \tau, \nabla_{x}\right)=D\left(-\nabla_{x}\right)^{\top} B(x, t, \tau) D\left(\nabla_{x}\right), \\
Q\left(x, t, \tau, \nabla_{x}\right)=D(n(x))^{\top} B(x, t, \tau) D\left(\nabla_{x}\right),
\end{array}\right.
$$

gives the operators with coefficients depending on the angular variable $\varphi_{\mathbf{i}} \in \Upsilon=(-\pi, \pi)$,

$$
\left\{\begin{aligned}
P^{\mathfrak{i}}\left(\varphi_{\mathfrak{i}}, t, \tau, \nabla_{x}\right) & =D\left(-\nabla_{x}\right)^{\top} B^{\mathfrak{i}}\left(\varphi_{\mathfrak{i}}, t, \tau\right) D\left(\nabla_{x}\right), \quad \varphi_{\mathfrak{i}} \in \Upsilon, \\
Q^{\mathfrak{i} \pm}\left(t, \tau, \nabla_{x}\right) & =D\left(\mp \mathbf{e}_{2}\right)^{\top} B^{\mathfrak{i}}( \pm \pi, t, \tau) D\left(\nabla_{x}\right),
\end{aligned}\right.
$$

with $B^{\mathfrak{i}}$ the "limit" of $B$ as $x \rightarrow \mathcal{O}^{\mathfrak{i}}$ in the sense of (1.9). Recall that for $B$ with smooth coefficients in the sense of (1.10), the coefficients in (5.2) are independent of $\varphi_{\mathbf{i}}$.

Similarly to (4.4), we write $P^{\mathfrak{i}}, Q^{\mathbf{i} \pm}$ in polar coordinates $(r, \varphi)$

$$
\left\{\begin{aligned}
P^{\mathfrak{i}}\left(\varphi, t, \tau, \nabla_{x}\right) & =: r^{-2} \mathcal{P}^{\mathfrak{i}}\left(t, \tau, \varphi, \partial_{\varphi}, r \partial_{r}\right), \\
Q^{\mathfrak{i} \pm}\left(t, \tau, \nabla_{x}\right) & =: r^{-1} \mathcal{Q}^{\mathfrak{i} \pm}\left(t, \tau, \partial_{\varphi}, r \partial_{r}\right) .
\end{aligned}\right.
$$

We fix one crack tip $\mathcal{O} \in\left\{\mathcal{O}^{\mathbf{I}}, \mathcal{O}^{\mathbf{I I}}\right\}$ and we therefore omit the index $\mathfrak{i} \in\{\mathbf{I}, \mathbf{I I}\}$. The time $t \in[0, T]$ is also fixed. Nevertheless, $t$ acts as a parameter and we will keep in our notations the dependency of the operators on $t$.

\subsection{Logarithmic right hand sides and solutions}

Let us fix $\lambda_{0} \in \Sigma, \lambda_{0}>0$. Let us go back to the iterative procedure used in the proof of Theorem 2.1: it consists in alternating the solution of an instantaneous problem $\left\{L, N^{ \pm}\right\}(t)$ with the application of the Volterra kernel $\left\{P, Q^{ \pm}\right\}(t, \tau)$. The Volterra kernel transforms a singularity of the form $r^{\lambda_{0}} \mathcal{U}^{0}(\varphi)$ into a right hand side of the form $\left\{r^{\lambda_{0}-2} \mathcal{F}^{0}(\varphi), r^{\lambda_{0}-1} \mathcal{G}^{0 \pm}\right\}$, corresponding to a Mellin transform with a pole of order 1 in $\lambda_{0}$. Then the next solution of the instantaneous problem will correspond to a Mellin transform with a pole of (generic) order 2, giving as singular part a term with a logarithm of the form $r^{\lambda_{0}}\left(\mathcal{U}^{0}(\varphi)+(\ln r) \mathcal{U}^{1}(\varphi)\right)$.

That is the reason why it is natural to consider right hand sides which have themselves a power logarithmic asymptotics. We first give simple formulas concerning the Mellin transform of functions with a power logarithmic expansion. 
Lemma 5.1. Let $\lambda_{0} \in \mathbb{R}, q \in \mathbb{N}_{0}$ and let be given $\mathcal{V}^{j} \in H^{\ell+1}(\Upsilon)$ for $j=0, \ldots, q$. We define the power logarithmic function $V$ as

$$
V(x)=r^{\lambda_{0}} \sum_{j=0}^{q} \frac{1}{j !}(\ln r)^{j} \mathcal{V}^{j}(\varphi)
$$

and the meromorphic function $\mathcal{V}$ as

$$
\mathcal{V}(\lambda)=\sum_{j=0}^{q} \frac{\mathcal{V}^{j}}{\left(\lambda-\lambda_{0}\right)^{j+1}}
$$

(i) We have the Cauchy residue formula for any $\varrho>0$ :

$$
V=\frac{1}{2 i \pi} \int_{\left|\lambda-\lambda_{0}\right|=\varrho} r^{\lambda} \mathcal{V}(\lambda) \mathrm{d} \lambda .
$$

(ii) Let us denote the characteristic function of the region $\mathbb{K}_{1}=\{x \in \mathbb{K}: r<1\}$ by $\Theta_{1}$. Let $\beta$ be such that $\ell-\beta<\lambda_{0}$. Then the function $\Theta_{1} V$ belongs to the weighted space $V_{\beta}^{\ell+1}\left(\mathbb{K}_{1}\right)$ and its norm satisfies the estimate:

$$
\left\|\Theta_{1} V\right\|_{V_{\beta}^{\ell+1}\left(\mathbb{K}_{1}\right)} \leq C \sum_{j=0}^{q} \frac{d^{j}}{\left(\lambda_{0}-l+\beta\right)^{j+1}}\left\|\mathcal{V}^{j}\right\|_{H^{\ell+1}(\Upsilon)}
$$

where $C>0$ and $d>1$ depend only on $l$.

(iii) Let $\gamma$ be such that $\ell-\gamma>\lambda_{0}$. Then the function $\left(1-\Theta_{1}\right) V$ belongs to $V_{\gamma}^{\ell+1}\left(\mathbb{K} \backslash \mathbb{K}_{1}\right)$ and its norm satisfies the estimate:

$$
\left\|\left(1-\Theta_{1}\right) V\right\|_{V_{\gamma}^{\ell+1}\left(\mathbb{K}_{1}\right)} \leq C \sum_{j=0}^{q} \frac{d^{j}}{\left(\ell-\gamma-\lambda_{0}\right)^{j+1}}\left\|\mathcal{V}^{j}\right\|_{H^{\ell+1}(\Upsilon)}
$$

(iv) The Mellin transform of $\Theta_{1} V$ is defined for all $\operatorname{Re} \lambda<\lambda_{0}$ whereas the Mellin transform of $\left(1-\Theta_{1}\right) V$ is defined for all $\operatorname{Re} \lambda>\lambda_{0}$ and both coincide with $\mathcal{V}$ in (5.5).

Proof. It relies on simple explicit computations based on the formula:

$$
\forall \varkappa>0, \quad \forall n \in \mathbb{N}_{0}, \quad \int_{0}^{1} r^{\varkappa}(\ln r)^{n} \frac{\mathrm{d} r}{r}=(-1)^{n} n ! \varkappa^{-n-1} .
$$

Let $\chi \in \mathcal{C}_{0}^{\infty}\left(\mathbb{R}^{2}\right)$ be a cut-off function which equals to 1 for $r<\frac{1}{2}$ and to 0 for $r>1$. We are ready for solving the model problem (4.3) with power logarithmic right-hand side. We will provide universal estimates for its solution (i.e. independent of its degree).

Proposition 5.2. Let $\lambda_{0} \neq 0$ belong to $\Sigma$. Let $\gamma$ and $\beta$ such that $\ell-\beta<\lambda_{0}<\ell-\gamma$ and $[\ell-\beta, \ell-\gamma] \cap \Sigma=\left\{\lambda_{0}\right\}$. Let $q \in \mathbb{N}_{0}$ and $\left\{\mathcal{F}^{j}, \mathcal{G}^{j \pm}\right\} \in R^{\ell} H(\Upsilon)$ for $j=0, \ldots, q-1$ and set

$$
H(x)=\sum_{j=0}^{q-1} \frac{1}{j !}(\ln r)^{j}\left\{r^{\lambda_{0}-2} \mathcal{F}^{j}, r^{\lambda_{0}-1} \mathcal{G}^{j \pm}\right\}, \quad x \in \mathbb{K} .
$$

(i) The right-hand side $\left\{F, G^{ \pm}\right\}=\chi H$ belongs to $R_{\beta}^{\ell} V(\mathbb{K})$ and problem (4.3) at time $t$ has a unique solution $U_{\beta} \in V_{\beta}^{\ell+1}(\mathbb{K})^{2}$. Similarly problem (4.3) at time $t$ with right hand side $\left\{F, G^{ \pm}\right\}=(1-\chi) H$ has a unique solution $U_{\gamma} \in V_{\gamma}^{\ell+1}(\mathbb{K})^{2}$. Moreover there exist $C_{0}>0$ and $\delta_{0}>1$ independent of $H, q$ and $t \in[0, T]$ so that

$$
\left\|U_{\beta}\right\|_{V_{\beta}^{\ell+1}(\mathbb{K})}+\left\|U_{\gamma}\right\|_{V_{\gamma}^{\ell+1}(\mathbb{K})} \leq C_{0} \sum_{j=0}^{q-1} \delta_{0}^{j}\left\|\left\{\mathcal{F}^{j}, \mathcal{G}^{j \pm}\right\}\right\|_{R^{\ell} H(\Upsilon)} .
$$


(ii) There exists unique angular functions $\mathcal{U}^{j} \in H^{\ell+1}(\Upsilon)^{2}, j=0, \ldots, q$ such that

$$
U_{\beta}=U_{\gamma}+r^{\lambda_{0}} \sum_{j=0}^{q} \frac{1}{j !}(\ln r)^{j} \mathcal{U}^{j}
$$

and we have the following estimates: for any $\delta_{1}>2$, there exists $C_{1}>0$ independent of $H, q$ and $t \in[0, T]$, so that there holds

$$
\max _{0 \leq j \leq q} \delta_{1}^{j}\left\|\mathcal{U}^{j}\right\|_{H^{\ell+1}(\Upsilon)} \leq C_{1} \max _{0 \leq j \leq q-1} \delta_{1}^{j}\left\|\left\{\mathcal{F}^{j}, \mathcal{G}^{j \pm}\right\}\right\|_{R^{\ell} H(\Upsilon)} .
$$

Proof.

(i) A straightforward modification of Lemma 5.1 (i) and (ii) yields that $\chi H$ belongs to $R_{\beta}^{\ell} V(\mathbb{K})$ and $(1-\chi) H$ to $R_{\gamma}^{\ell} V(\mathbb{K})$ with the estimates

$$
\|\chi H\|_{R_{\beta}^{\ell} V(\mathbb{K})}+\|(1-\chi) H\|_{R_{\gamma}^{\ell} V(\mathbb{K})} \leq C \sum_{j=0}^{q-1} \delta_{0}^{j}\left\|\left\{\mathcal{F}^{j}, \mathcal{G}^{j \pm}\right\}\right\|_{R^{\ell} H(\Upsilon)} .
$$

Using the continuity of the inverse of the operator of problem (4.3) in the suitable spaces, compare with Theorem 4.2, we obtain (5.9). The assumptions (1.5) of uniform ellipticity and (1.6) of boundedness yield that the constant $C_{0}$ in (5.9) can be chosen independently of $t \in[0, T]$.

(ii) Let us define $\mathcal{H}(\lambda)$ as the Mellin transform of $\chi\left\{r^{2} F, r G^{ \pm}\right\}$:

$$
\mathcal{H}(\lambda)=\left\{\mathcal{M}\left[\chi r^{2} F\right], \mathcal{M}\left[\chi r G^{ \pm}\right]\right\}(\lambda) \quad \text { for } \quad \operatorname{Re} \lambda<\lambda_{0} .
$$

We note that $\left\{F_{\text {reg }}, G_{\text {reg }}^{ \pm}\right\}:=\chi H-\Theta_{1} H$ has its support in the region $\left\{x: \frac{1}{2}<r<1\right\}$. Therefore the Mellin transform

$$
\mathcal{H}_{\text {reg }}(\lambda)=\left\{\mathcal{M}\left[r^{2} F_{\text {reg }}\right], \mathcal{M}\left[r G_{\text {reg }}^{ \pm}\right]\right\}(\lambda)
$$

is holomorphic in the whole complex plane and easy computations yield that $\mathcal{H}_{\text {reg }}$ satisfies the following estimates in any disc $\left\{\lambda \in \mathbb{C}:\left|\lambda-\lambda_{0}\right| \leq \varrho\right\}$ :

$$
\left\|\mathcal{H}_{\mathrm{reg}}(\lambda)\right\|_{R^{\ell} H(\Upsilon)} \leq C_{\varrho}^{\prime} \sum_{j=0}^{q-1} \frac{1}{j !}\left\|\left\{\mathcal{F}^{j}, \mathcal{G}^{j \pm}\right\}\right\|_{R^{\ell} H(\Upsilon)},
$$

with a constant $C_{\varrho}^{\prime}>0$ depending only on $l$ and $\varrho$. We deduce from (5.5) that

$$
\mathcal{H}(\lambda)=\mathcal{H}_{\mathrm{reg}}(\lambda)+\sum_{j=0}^{q-1} \frac{\mathcal{H}^{j}}{\left(\lambda-\lambda_{0}\right)^{j+1}} \quad \text { with } \quad \mathcal{H}^{j}:=\left\{\mathcal{F}^{j}, \mathcal{G}^{j \pm}\right\}
$$

By (4.11) we obtain the expansion (5.10) together with the Cauchy formula,

$$
r^{\lambda_{0}} \sum_{j=0}^{q} \frac{1}{j !}(\ln r)^{j} \mathcal{U}^{j}=\frac{1}{2 i \pi} \int_{\left|\lambda-\lambda_{0}\right|=\varrho} r^{\lambda} \mathcal{A}[t](\lambda)^{-1} \mathcal{H}(\lambda) \mathrm{d} \lambda .
$$

Combining with the Cauchy formula (5.6) we obtain

$$
\sum_{j=0}^{q} \frac{\mathcal{U}^{j}}{\left(\lambda-\lambda_{0}\right)^{j+1}} \simeq \mathcal{A}[t](\lambda)^{-1}\left(\sum_{n=0}^{q-1} \frac{\mathcal{H}^{n}}{\left(\lambda-\lambda_{0}\right)^{n+1}}+\mathcal{H}_{\mathrm{reg}}\left(\lambda_{0}\right)\right)
$$


modulo holomorphic functions. The Laurent expansion of $\mathcal{A}[t](\lambda)^{-1}$ at $\lambda_{0}$ takes the form of the convergent series:

$$
\mathcal{A}[t](\lambda)^{-1}=\frac{\mathcal{R}_{-1}[t]}{\lambda-\lambda_{0}}+\sum_{m \geq 0}^{\infty} \mathcal{R}_{m}[t]\left(\lambda-\lambda_{0}\right)^{m} .
$$

The radius of convergence of this series is the distance to the closest pole to $\lambda_{0}$. This distance being $\frac{1}{2}$, the radius of convergence is $\frac{1}{2}$, which means that for all $\delta>2$, we have the estimate for the terms $\mathcal{R}_{m}[t]$ with a constant $C=C(\delta, \mathcal{A}[t])$

$$
\left\|\mathcal{R}_{m}[t]\right\|_{R^{\ell} H(\Upsilon) \rightarrow H^{\ell+1}(\Upsilon)} \leq C \delta^{m}, \quad m=-1,0, \ldots
$$

Combining (5.15) with (5.16) and identifying the powers of $\left(\lambda-\lambda_{0}\right)$, we obtain

$$
\mathcal{U}^{j}=\sum_{\substack{n-m=j \\ n \geq-1, m \geq-1}} \mathcal{R}_{m}[t] \mathcal{H}^{n}
$$

with the convention $\mathcal{H}^{-1}:=\mathcal{H}_{\text {reg }}\left(\lambda_{0}\right)$.

Let us choose $\delta_{1}>2$ and let us denote by $\mathbf{N}$ the bound $\max _{j=0, \ldots, q-1} \delta_{1}^{j}\left\|\mathcal{H}^{j}\right\|_{R^{\ell} H(\Upsilon)}$. By definition,

$$
\left\|\mathcal{H}^{j}\right\|_{R^{\ell} H(\Upsilon)} \leq \delta_{1}^{-j} \mathbf{N}, \quad j=0, \ldots, q-1 .
$$

Thus (5.12) yields for $\mathcal{H}^{-1}=\mathcal{H}_{\text {reg }}\left(\lambda_{0}\right)$ :

$$
\left\|\mathcal{H}^{-1}\right\|_{R^{\ell} H(\Upsilon)} \leq C^{\prime} \mathrm{e}^{-\delta_{1}} \mathbf{N} \leq C^{\prime} \delta_{1} \mathbf{N},
$$

which means that we have finally

$$
\left\|\mathcal{H}^{j}\right\|_{R^{\ell} H(\Upsilon)} \leq C^{\prime} \delta_{1}^{-j} \mathbf{N}, \quad j=-1,0, \ldots, q-1 .
$$

Therefore estimates (5.17) and (5.19) yield

$$
\begin{aligned}
\left\|\mathcal{U}^{j}\right\|_{H^{\ell+1}(\Upsilon)} & \leq \sum_{n-m=j}\left\|\mathcal{R}_{m}[t]\right\|_{R^{\ell} H(\Upsilon) \rightarrow H^{\ell+1}(\Upsilon)}\left\|\mathcal{H}^{n}\right\|_{R^{\ell} H(\Upsilon)} \\
& \leq \sum_{n-m=j} C C^{\prime} \delta^{m} \mathbf{N} \delta_{1}^{-n}=\sum_{n-m=j} C C^{\prime}\left(\frac{\delta}{\delta_{1}}\right)^{m} \mathbf{N} \delta_{1}^{-j} \\
& \leq C C^{\prime} \mathbf{N} \delta_{1}^{-j} \sum_{m=-1}^{\infty}\left(\frac{\delta}{\delta_{1}}\right)^{m}=C C^{\prime} \mathbf{N} \delta_{1}^{-j} \frac{\delta_{1}}{\delta} \frac{1}{1-\delta / \delta_{1}} .
\end{aligned}
$$

Choosing $\delta \in\left(2, \delta_{1}\right)$ and letting

$$
C_{1}=C(\delta, \mathcal{A}[t]) C^{\prime} \delta_{1} \delta^{-1}\left(1-\delta / \delta_{1}\right)^{-1}
$$

we deduce (5.11) from (5.20). The assumptions (1.5)-(1.6) give us that the constants $C(\delta, \mathcal{A}[t])$ are bounded in $t$, therefore the constant $C_{1}$ in (5.11) can be chosen independently of $t \in[0, T]$, once $\delta_{1}>2$ is chosen.

Remark 5.3. The identity: $\mathcal{A}[t](\lambda)^{-1} \mathcal{A}[t](\lambda)=\mathrm{Id}$ combined with expansion (5.16), gives by identifying the coefficients of $\left(\lambda-\lambda_{0}\right)^{-1}$

$$
\mathcal{R}_{-1}[t] \mathcal{A}[t]\left(\lambda_{0}\right)=0, \quad \text { i.e. } \quad \mathcal{R}_{-1}[t] \equiv 0 \quad \text { on } \quad \text { Range } \mathcal{A}[t]\left(\lambda_{0}\right) .
$$

We note that the augmentation of the logarithmic degree from $H$ to $U$ in Proposition 5.2 comes from the only term $\mathcal{R}_{-1}[t] \mathcal{H}^{q-1}$. Thus if $\mathcal{H}^{q-1}$ belongs to the range of $\mathcal{A}[t]\left(\lambda_{0}\right)$, the logarithmic degree does not increase. 
It remains to give an estimate of the action of the relaxation kernel frozen in $\mathcal{O}$ over a power-logarithmic expression.

Proposition 5.4. Let $\lambda_{0} \in \mathbb{R}, q \in \mathbb{N}_{0}$ and let be given $\mathcal{U}^{j} \in H^{\ell+1}(\Upsilon)$ for $j=0, \ldots, q$. We define the power logarithmic function $\left\{F, G^{ \pm}\right\}$by

$$
\left\{P\left(\varphi, t, \tau, \nabla_{x}\right), Q^{ \pm}\left(t, \tau, \nabla_{x}\right)\right\}\left(r^{\lambda_{0}} \sum_{j=0}^{q} \frac{1}{j !}(\ln r)^{j} \mathcal{U}^{j}(\varphi)\right)=r^{\lambda_{0}} \sum_{j=0}^{q} \frac{1}{j !}(\ln r)^{j}\left\{r^{-2} \mathcal{F}^{j}(t, \tau, \varphi), r^{-1} \mathcal{G}^{j \pm}(t, \tau)\right\}
$$

For any $\delta>0$, there exists $C>0$ independent of $\mathcal{U}^{j}, q$ and $(t, \tau) \in \mathcal{T}(T)$, so that there holds

$$
\max _{0 \leq j \leq q} \delta^{j}\left\|\left\{\mathcal{F}^{j}, \mathcal{G}^{j \pm}\right\}(t, \tau)\right\|_{R^{\ell} H(\Upsilon)} \leq C \max _{0 \leq j \leq q} \delta^{j}\left\|\mathcal{U}^{j}\right\|_{H^{\ell+1}(\Upsilon)}
$$

Proof. Let $\mathcal{B}[t, \tau](\lambda):=\left\{\mathcal{P}\left(t, \tau, \varphi, \partial_{\varphi}, \lambda\right), \mathcal{Q}^{ \pm}\left(t, \tau, \partial_{\varphi}, \lambda\right)\right\}$ be the Mellin symbol of the expression (5.3) of the frozen relaxation kernel in polar coordinates. We have the formula, compare with (5.15)

$$
\mathcal{B}[t, \tau](\lambda)\left(\sum_{j=0}^{q} \frac{\mathcal{U}^{j}}{\left(\lambda-\lambda_{0}\right)^{j+1}}\right)=\sum_{j=0}^{q} \frac{\left\{\mathcal{F}^{j}, \mathcal{G}^{j \pm}\right\}(t, \tau)}{\left(\lambda-\lambda_{0}\right)^{j+1}}
$$

But, since $\mathcal{P}$ and $\mathcal{Q}^{ \pm}$are differential operators in $r \partial_{r}$ of order $\leq 2$, we have the expansion

$$
\mathcal{B}[t, \tau](\lambda)=\mathcal{B}^{0}[t, \tau]+\mathcal{B}^{1}[t, \tau]\left(\lambda-\lambda_{0}\right)+\mathcal{B}^{2}[t, \tau]\left(\lambda-\lambda_{0}\right)^{2}
$$

and, therefore:

$$
\left\{\mathcal{F}^{j}, \mathcal{G}^{j \pm}\right\}(t, \tau)=\mathcal{B}^{0}[t, \tau] \mathcal{U}^{j}+\mathcal{B}^{1}[t, \tau] \mathcal{U}^{j-1}+\mathcal{B}^{2}[t, \tau] \mathcal{U}^{j-2}
$$

The uniform bound (1.7) on the relaxation kernel yields a uniform bound on the operators $\mathcal{B}^{k}, k=0,1,2$, as continuous operators $H^{\ell+1}(\Upsilon) \rightarrow R^{\ell} H(\Upsilon)$. Hence estimate (5.23) follows.

\subsection{Absence of logarithms: Cayley representation}

In the situation where the limit "stabilized" material laws $B^{\mathfrak{i}}(t, \tau)$ for $\mathfrak{i}=\mathbf{I}$, II, of the relaxation kernel are independent of the angular variable $\varphi$, we are going to show that the iterative procedure (3.18)-(3.19) produces singularities of the form $r^{n+1 / 2} \mathcal{V}(\varphi)$ only, with the exclusion of any logarithmic term. As usual, we fix a crack tip $\mathcal{O}^{\mathfrak{i}}$ and omit its mention.

In order prove this "non appearance of logarithms", for any fixed non integer element $\lambda_{0}$ of $\Sigma$, we construct

- a class $\mathfrak{U}\left(\lambda_{0}\right)$ of functions $U(x)$ homogeneous of degree $\lambda_{0}$; i.e. of the form $r^{\lambda_{0}} \mathcal{U}(\varphi)$;

- a class $\mathfrak{H}\left(\lambda_{0}\right)$ of corresponding right hand sides $H(x)=\left\{F, G^{ \pm}\right\}(x)$,

with the following three properties:

(i) for any $t \in(0, T)$, the primal singular functions (4.12) belong to $\mathfrak{U}\left(\lambda_{0}\right)$;

(ii) if the frozen Volterra kernel $\left\{P, Q^{ \pm}\right\}(t, \tau)$ has constant coefficients in $\varphi$, then for all $U \in \mathfrak{U}\left(\lambda_{0}\right)$, $\left\{P, Q^{ \pm}\right\}(t, \tau) U$ belongs to $\mathfrak{H}\left(\lambda_{0}\right)$

(iii) for any $H \in \mathfrak{H}\left(\lambda_{0}\right)$, the solution (5.4) belongs to $\mathfrak{U}\left(\lambda_{0}\right)$.

We can see that, as a result, the singularities of the terms $u^{k}, k=0,1, \ldots$, generated by the iterative procedure (3.18)-(3.19) will stay inside $\mathfrak{U}\left(\lambda_{0}\right)$, excluding the appearance of any logarithmic term.

We start with the definition of a class $\mathfrak{U}(\lambda)$ for each $\lambda$ in the disc $D\left(\lambda_{0}, \frac{1}{4}\right)$. Let

$$
\zeta:=r \mathrm{e}^{i \varphi}, \quad r>0, \quad \varphi \in(-\pi, \pi)
$$


be the complex writing of the coordinates $x \in \mathbb{K}$ centered at $\mathcal{O}$. We have to define an Ansatz for an admissible homogeneous function of degree $\lambda$. The prototypes are the functions $\zeta^{\lambda}$ and $\bar{\zeta}^{\lambda}$, which are sufficient to describe the singularities of the Laplace operator. For a wider class of scalar elliptic operators, they have to be generalized to $(\alpha \zeta+\bar{\zeta})^{\lambda}$ and $(\zeta+\alpha \bar{\zeta})^{\lambda}$ with $\alpha$ a complex parameter of modulus $<1$, and finally, to cover any AgmonDouglis-Nirenberg system, to contour integrals in $\alpha$ of such functions [8].

We give the following meaning to $(\alpha \zeta+\bar{\zeta})^{\lambda}$ and $(\zeta+\alpha \bar{\zeta})^{\lambda}$ for $|\alpha|<1$ and $\zeta \in \mathbb{K}$ :

$$
(\alpha \zeta+\bar{\zeta})^{\lambda}:=\bar{\zeta}^{\lambda}\left(1+\alpha \frac{\zeta}{\bar{\zeta}}\right)^{\lambda} \text { and }(\zeta+\alpha \bar{\zeta})^{\lambda}:=\zeta^{\lambda}\left(1+\alpha \frac{\bar{\zeta}}{\zeta}\right)^{\lambda}
$$

The functions $\zeta^{\lambda}$ and $\bar{\zeta}^{\lambda}$ are well defined on $\mathbb{K}$ and as $|\alpha|<1$, the functions $1+\alpha \zeta / \bar{\zeta}$ and $1+\alpha \bar{\zeta} / \zeta$ take their values in the half plane $\operatorname{Re} z>0$, thus the products in (5.27) make sense.

For any fixed time $t$, the homogeneous operator $L\left(t, \nabla_{x}\right)$, see (4.2), transforms the homogeneous functions (5.27) in similar functions with $\lambda-2$ instead $\lambda$ : there holds

$$
\begin{aligned}
& L\left(t, \nabla_{x}\right)(\alpha \zeta+\bar{\zeta})^{\lambda}=\lambda(\lambda-1)(\alpha \zeta+\bar{\zeta})^{\lambda-2} L_{+}[t](\alpha), \\
& L\left(t, \nabla_{x}\right)(\zeta+\alpha \bar{\zeta})^{\lambda}=\lambda(\lambda-1)(\zeta+\alpha \bar{\zeta})^{\lambda-2} L_{-}[t](\alpha),
\end{aligned}
$$

where $L_{ \pm}[t](\alpha)$ are the Cayley symbols associated with $L\left(t, \nabla_{x}\right) \equiv L\left(t, \partial_{1}, \partial_{2}\right)$ :

$$
L_{+}[t](\alpha):=L(t, \alpha+1, i(\alpha-1)) \quad \text { and } \quad L_{-}[t](\alpha):=L(t, 1+\alpha, i(1-\alpha)) .
$$

Due to the uniform ellipticity condition (1.5) there exists $\varrho<1$ such that for all $t \in[0, T]$ and for all $\alpha \in \mathbb{C}$ with $|\alpha| \in[\varrho, 1]$,

$$
\operatorname{det} L_{ \pm}[t](\alpha) \neq 0
$$

Let us denote by $\mathfrak{A}$ the space of complex analytic functions of $\alpha$ in the ring $\{\alpha \in \mathbb{C}:|\alpha| \in[\varrho, 1]\}$. We set

$$
\begin{aligned}
\mathfrak{U}_{+}(\lambda) & =\left\{U: \exists \mathfrak{q}_{+} \in \mathfrak{A}^{2} \quad U=\int_{|\alpha|=\varrho}(\alpha \zeta+\bar{\zeta})^{\lambda} \mathfrak{q}_{+}(\alpha) \mathrm{d} \alpha\right\} \\
\mathfrak{U}_{-}(\lambda) & =\left\{U: \exists \mathfrak{q}_{-} \in \mathfrak{A}^{2} \quad U=\int_{|\alpha|=\varrho}(\zeta+\alpha \bar{\zeta})^{\lambda} \mathfrak{q}_{-}(\alpha) \mathrm{d} \alpha\right\} .
\end{aligned}
$$

Note that we can use any circle $|\alpha|=R$ with $\varrho \leq R<1$ in the above contour integrals without modifying the result.

Lemma 5.5. If $\lambda$ is not an integer, the intersection $\mathfrak{U}_{+}(\lambda) \cap \mathfrak{U}_{-}(\lambda)$ is reduced to $\{0\}$.

Proof. Let $U_{ \pm} \in \mathfrak{U}_{ \pm}(\lambda)$. According to the definition (5.27), we can write

$$
\begin{aligned}
& U_{+}(r, \varphi)=\int_{|\alpha|=\varrho}(\alpha \zeta+\bar{\zeta})^{\lambda} \mathfrak{q}_{+}(\alpha) \mathrm{d} \alpha=r^{\lambda} \mathrm{e}^{-i \lambda \varphi} \int_{|\alpha|=\varrho}\left(1+\alpha \mathrm{e}^{2 i \varphi}\right)^{\lambda} \mathfrak{q}_{+}(\alpha) \mathrm{d} \alpha \\
& U_{-}(r, \varphi)=\int_{|\alpha|=\varrho}(\zeta+\alpha \bar{\zeta})^{\lambda} \mathfrak{q}_{-}(\alpha) \mathrm{d} \alpha=r^{\lambda} \mathrm{e}^{i \lambda \varphi} \int_{|\alpha|=\varrho}\left(1+\alpha \mathrm{e}^{-2 i \varphi}\right)^{\lambda} \mathfrak{q}_{-}(\alpha) \mathrm{d} \alpha .
\end{aligned}
$$

We see that as functions of $\varphi$, both $U_{+}(r, \varphi)$ and $U_{-}(r, \varphi)$ have unique analytic continuations from $[-\pi, \pi]$ to all of $\mathbb{R}$ satisfying

$$
U_{+}(r, \varphi+\pi)=\mathrm{e}^{-i \lambda \pi} U_{+}(r, \phi) \quad \text { and } \quad U_{-}(r, \varphi+\pi)=\mathrm{e}^{i \lambda \pi} U_{-}(r, \phi) \quad \text { for all } \varphi \in \mathbb{R} .
$$


Now assume that $U_{+}=U_{-}$. By analyticity, this holds for all $\varphi \in \mathbb{R}$ if it holds for $\varphi$ in some non-trivial interval (in our case for $\varphi \in[-\pi, \pi]$ ). We find

$$
0=U_{+}(r, \varphi)-U_{-}(r, \varphi)=\left(\mathrm{e}^{-i \lambda \pi}-\mathrm{e}^{i \lambda \pi}\right) U_{+}(r, \varphi) \quad \text { for all } \varphi \in \mathbb{R} .
$$

Hence either $\lambda \in \mathbb{Z}$ or $U_{+}(r, \varphi)=0$ for all $\varphi \in \mathbb{R}$.

As a consequence of ([8], Thm. 2.1), the above Ansatz covers all solutions (4.8) of the homogeneous system (4.3) without boundary conditions:

Proposition 5.6. Let $\mathfrak{W}[t](\lambda)$ be the space of solutions (4.8) of the homogeneous system

$$
L\left(t, \nabla_{x}\right) U(x)=0, \quad x \in \mathbb{K} .
$$

Then for all $t \in(0, T)$ and non-integer $\lambda \in \mathbb{C}$

$$
\mathfrak{W}[t](\lambda) \subset \mathfrak{U}_{+}(\lambda) \oplus \mathfrak{U}_{-}(\lambda) .
$$

Furthermore, both spaces $\mathfrak{W}[t](\lambda) \cap \mathfrak{U}_{+}(\lambda)$ and $\mathfrak{W}[t](\lambda) \cap \mathfrak{U}_{-}(\lambda)$ have dimension 2 .

Since the order of the pole $\lambda_{0}$ of $\mathcal{A}[t](\lambda)^{-1}$ is 1 , the primal singularities in $\lambda_{0}$ are linear combinations of elements in $\mathfrak{W}[t]\left(\lambda_{0}\right)$. Therefore our first requirement (i) is met with

$$
\mathfrak{U}\left(\lambda_{0}\right):=\mathfrak{U}_{+}\left(\lambda_{0}\right) \oplus \mathfrak{U}_{-}\left(\lambda_{0}\right) .
$$

As already suggested by formulas (5.28) homogeneous operators with constant coefficients act between spaces $\mathfrak{U}(\lambda)$ with distinct $\lambda$.

Lemma 5.7. Let $P\left(\nabla_{x}\right)$ be a $2 \times 2$ matrix of differential operators of order $m$, homogeneous with constant coefficients. Then there holds $P U \in \mathfrak{U}(\lambda-m)$ for all $U \in \mathfrak{U}(\lambda)$.

Proof. The coefficients of $P\left(\nabla_{x}\right)$ are linear combinations of products of the form $\partial_{\zeta}^{\kappa_{1}} \partial_{\bar{\zeta}}^{\kappa_{2}}$ with $\kappa_{1}+\kappa_{2}=m$. However

$$
\partial_{\zeta}(\alpha \zeta+\bar{\zeta})^{\lambda}=\lambda \alpha(\alpha \zeta+\bar{\zeta})^{\lambda-1} \quad \text { and } \quad \partial_{\zeta}(\zeta+\alpha \bar{\zeta})^{\lambda}=\lambda(\zeta+\alpha \bar{\zeta})^{\lambda-1}
$$

and similarly for $\partial_{\bar{\zeta}}$. The conclusion of the lemma is now obvious.

Let us now define for $\lambda=\lambda_{0}$ the space $\mathfrak{H}\left(\lambda_{0}\right)$

$$
\mathfrak{H}\left(\lambda_{0}\right):=\left\{\left\{F, G^{ \pm}\right\}: F \in \mathfrak{U}\left(\lambda_{0}-2\right), G^{ \pm}=r^{\lambda_{0}-1} g^{ \pm}, g^{ \pm} \in \mathbb{C}, \text { with } g^{+}=g^{-}\right\} .
$$

Lemma 5.8. Let $P\left(\nabla_{x}\right)$ and $Q\left(\nabla_{x}\right)$ be $2 \times 2$ matrices of differential operators homogeneous of order 2 and 1 , respectively. Then there holds $\{P, \pm Q\} U \in \mathfrak{H}\left(\lambda_{0}\right)$ for all $U \in \mathfrak{U}\left(\lambda_{0}\right)$.

According to this lemma, our second requirement (ii) is met, since in the constant coefficient case for all $(t, \tau) \in \mathcal{T}(T), Q^{ \pm}(t, \tau)$ has the form $\pm Q$.

Proof. In view of Lemma 5.7, we only have to prove that $\left.Q U\right|_{\varphi=\pi}=-\left.Q U\right|_{\varphi=-\pi}$. Indeed, $Q U$ belongs to $\mathfrak{U}\left(\lambda_{0}-1\right)$. Let $\lambda_{1}:=\lambda_{0}-1$. Let us prove that

$$
\forall V \in \mathfrak{U}\left(\lambda_{1}\right),\left.\quad V\right|_{\varphi=\pi}=-\left.V\right|_{\varphi=-\pi} .
$$


In view of the Ansatz (5.30), it suffices to prove (5.33) for a scalar function $V$ of the form $(\alpha \zeta+\bar{\zeta})^{\lambda_{1}}$ or $(\zeta+\alpha \bar{\zeta})^{\lambda_{1}}$. We then use (5.27). We have

$$
\begin{aligned}
\left.\bar{\zeta}^{\lambda_{1}}\left(1+\alpha \frac{\zeta}{\bar{\zeta}}\right)^{\lambda_{1}}\right|_{\varphi=\pi}=\left.r \mathrm{e}^{-i \lambda_{1} \varphi}\left(1+\alpha \mathrm{e}^{2 i \varphi}\right)^{\lambda_{1}}\right|_{\varphi=\pi} & =r \mathrm{e}^{-i \lambda_{1} \pi}(1+\alpha)^{\lambda_{1}} \\
\text { and }\left.\quad \bar{\zeta}^{\lambda_{1}}\left(1+\alpha \frac{\zeta}{\bar{\zeta}}\right)^{\lambda_{1}}\right|_{\varphi=-\pi} & =r \mathrm{e}^{+i \lambda_{1} \pi}(1+\alpha)^{\lambda_{1}}
\end{aligned}
$$

Since $\lambda_{1}=m+\frac{1}{2}$, with an integer $m, \mathrm{e}^{ \pm i \lambda_{1} \pi}= \pm i$. Whence (5.33) for $V=(\alpha \zeta+\bar{\zeta})^{\lambda_{1}}$. The proof for the other case is similar.

We end this subsection with the proof of our third requirement (iii):

Lemma 5.9. Let $H=\left\{F, G^{ \pm}\right\}$belong to $\mathfrak{H}\left(\lambda_{0}\right)$. Then there exists a solution $U \in \mathfrak{U}\left(\lambda_{0}\right)$ of the model problem (4.3): $\left\{L\left(t, \nabla_{x}\right), N^{ \pm}\left(t, \nabla_{x}\right)\right\} U=\left\{F, G^{ \pm}\right\}$.

Proof. By assumption there exist $\mathfrak{q}_{ \pm} \in \mathfrak{A}^{2}$ such that

$$
F=\int_{|\alpha|=\varrho}(\alpha \zeta+\bar{\zeta})^{\lambda_{0}-2} \mathfrak{q}_{+}(\alpha) \mathrm{d} \alpha+\int_{|\alpha|=\varrho}(\zeta+\alpha \bar{\zeta})^{\lambda_{0}-2} \mathfrak{q}_{-}(\alpha) \mathrm{d} \alpha .
$$

Therefore by setting - here we use the Cayley symbols (5.29),

$$
\begin{aligned}
U_{0}= & \frac{1}{\lambda_{0}\left(\lambda_{0}-1\right)} \int_{|\alpha|=\varrho}(\alpha \zeta+\bar{\zeta})^{\lambda_{0}} L_{+}[t](\alpha)^{-1} \mathfrak{q}_{+}(\alpha) \mathrm{d} \alpha \\
& +\frac{1}{\lambda_{0}\left(\lambda_{0}-1\right)} \int_{|\alpha|=\varrho}(\zeta+\alpha \bar{\zeta})^{\lambda_{0}} L_{-}[t](\alpha)^{-1} \mathfrak{q}_{-}(\alpha) \mathrm{d} \alpha
\end{aligned}
$$

we obtain $U_{0} \in \mathfrak{U}\left(\lambda_{0}\right)$ such that $L\left(t, \nabla_{x}\right) U_{0}=F$. Lemma 5.8 yields that $\left\{L, N^{ \pm}\right\} U_{0}$ (which is equal to $\left.\{L, \pm N\} U_{0}\right)$ belongs to $\mathfrak{H}\left(\lambda_{0}\right)$. Considering the new right hand side $H-\left\{L, N^{ \pm}\right\} U_{0}$, we are reduced to solve the model problem (4.3) with $F=0$ and the compatibility condition $G^{+}=G^{-}$. Let us consider the mapping $\mathfrak{N}\left(\lambda_{0}\right)$ defined on the spaces $\mathfrak{W}$ of Proposition 5.6

$$
\begin{aligned}
\mathfrak{W}\left(\lambda_{0}\right) & \longrightarrow \mathbb{C}^{2} \times \mathbb{C}^{2} \\
U & \longmapsto\left(\left.N^{+} U\right|_{r=1, \varphi=\pi},\left.N^{-} U\right|_{r=1, \varphi=-\pi}\right) .
\end{aligned}
$$

As $\mathfrak{W}\left(\lambda_{0}\right) \subset \mathfrak{U}\left(\lambda_{0}\right)$, Lemma 5.8 yields that the range of $\mathfrak{N}\left(\lambda_{0}\right)$ is contained in the two-dimensional space $\left\{\left(g^{+}, g^{-}\right) \in \mathbb{C}^{2} \times \mathbb{C}^{2}: g^{+}=g^{-}\right\}$. The kernel of $\mathfrak{N}\left(\lambda_{0}\right)$ is the space of singular functions homogeneous of degree $\lambda_{0}$. This space is of dimension 2 (see Lem. 4.4 for $\lambda_{0}=\frac{1}{2}$, and [9] for more general framework). Therefore the range of $\mathfrak{N}\left(\lambda_{0}\right)$ is the whole space $\left\{\left(g^{+}, g^{-}\right) \in \mathbb{C}^{2} \times \mathbb{C}^{2}: g^{+}=g^{-}\right\}$, and there exists a solution in $\mathfrak{W}\left(\lambda_{0}\right) \subset \mathfrak{U}\left(\lambda_{0}\right)$ to our last problem.

As a consequence of Lemma 5.5 and the symbolic calculus of Lemmas 5.8 and 5.9, we obtain immediately the following result.

Proposition 5.10. Let $\lambda_{0} \in \Sigma \backslash \mathbb{Z}$. The spaces $\mathfrak{U}\left(\lambda_{0}\right)$ and $\mathfrak{H}\left(\lambda_{0}\right)$, defined in (5.30)-(5.31) and (5.32), respectively, equipped with the norms

$$
\begin{aligned}
\|U\|_{\mathfrak{U}\left(\lambda_{0}\right)} & =\max _{\varrho \leq|\alpha| \leq 1}\left|\mathfrak{q}_{+}(\alpha)\right|+\max _{\varrho \leq|\alpha| \leq 1}\left|\mathfrak{q}_{-}(\alpha)\right|, \\
\left\|\left\{F, r^{\lambda_{0}-1} g^{ \pm}\right\}\right\|_{\mathfrak{H}\left(\lambda_{0}\right)} & =\|F\|_{\mathfrak{U}\left(\lambda_{0}-2\right)}+\left|g^{+}\right|+\left|g^{-}\right|
\end{aligned}
$$


are Banach spaces. Moreover, the operators

$$
\begin{aligned}
\left\{P\left(t, \tau, \nabla_{x}\right), Q^{ \pm}\left(t, \tau, \nabla_{x}\right)\right\} \quad \text { and } & \left\{L\left(t, \nabla_{x}\right), N^{ \pm}\left(t, \nabla_{x}\right)\right\} \quad: \mathfrak{U}\left(\lambda_{0}\right) \rightarrow \mathfrak{H}\left(\lambda_{0}\right) \\
& \left\{L\left(t, \nabla_{x}\right), N^{ \pm}\left(t, \nabla_{x}\right)\right\}^{-1}: \mathfrak{H}\left(\lambda_{0}\right) \rightarrow \mathfrak{U}\left(\lambda_{0}\right)
\end{aligned}
$$

are bounded linear operators whose norms depend only on bounds of the coefficients of the differential operators, cf. (1.6)-(1.7) and, concerning $\left\{L\left(t, \nabla_{x}\right), N^{ \pm}\left(t, \nabla_{x}\right)\right\}^{-1}$, on the ellipticity constant $c_{A}(1.5)$ of the operator $A$.

\section{Asymptotics NeAR the CRACK tips FOR THE CREeP PROBlem}

We come back to the solution $u(x, t), x \in \Omega, t \in(0, T)$, of the creep problem (1.13). We make the following assumption on the data $\{f(t), g(t)\}$ :

$$
\{f, g\} \in L_{\infty}\left(0, T ; R_{\gamma}^{\ell, 1} V(\Omega)\right), \quad \gamma \text { such that } \ell-\gamma \in\left(1, \frac{3}{2}\right)
$$

with the compatibility condition (2.7). Here $l$ is the positive integer in (1.6)-(1.7) and $R_{\gamma}^{\ell, 1} H(\Omega)$ is the space (4.32), where the tractions $g$ are supposed to satisfy the compatibility conditions $g^{+}\left(\mathcal{O}^{\mathfrak{i}}\right)+g^{-}\left(\mathcal{O}^{\mathfrak{i}}\right)=0$ at the crack tips. We will discuss later on the more general case when $\{f, g\} \in L_{\infty}\left(0, T ; R_{\gamma}^{\ell} H(\Omega)\right)$, where no condition on the traces $g^{ \pm}\left(\mathcal{O}^{\mathfrak{i}}\right)$ are prescribed. We assume that the stabilization rate in (1.9) satisfies the inequality

$$
\delta_{B}>\ell-\gamma-1 / 2
$$

where, since $\ell-\gamma>1$, the lower bound is larger than $1 / 2$.

From Theorem 3.5, we know that the solution $u$ belongs to $L_{\infty}\left(0, T ; V_{\beta}^{\ell+1}(\Omega)\right)^{2}$ for all $\beta$ such that $\ell-\beta \in$ $\left(0, \frac{1}{2}\right)$. We recall that $u$ has the representation (3.18) as a sum of terms $u^{k}$ which solve of the problems $\{L, N\} u^{k}=\left\{f^{k}, g^{k}\right\}$ subject to the orthogonality condition (2.7). The right hand sides $\left\{f^{k}, g^{k}\right\}$ are given by $\left\{f^{0}, g^{0}\right\}=\{f, g\}$ for $k=0$ and (3.19) for $k \in \mathbb{N}$.

In this section we are going to derive the following asymptotic formulae for the members $u^{k}$ of series (3.18) (the "iterate solutions"):

$$
u^{k}(x, t)=\widetilde{u}^{k}(x, t)+\sum_{\mathfrak{i}=\mathbf{I}, \mathbf{I I}} \chi^{\mathfrak{i}}(x)\left(a_{\mathfrak{i}}^{k}(t)+V_{\mathfrak{i}}^{k}(x, t)+W_{\mathfrak{i}}^{k}(x, t)\right),
$$

where $\widetilde{u}^{k}$ is the "flat regular part" in $L_{\infty}\left(0, T ; V_{\gamma}^{\ell+1}(\Omega)\right)^{2}$, the terms $a_{\mathfrak{i}}^{k}(t)$ are constants in $\mathbb{R}^{2}$ at each fixed time, and the functions $V_{\mathfrak{i}}^{k}$ and $W_{\mathfrak{i}}^{k}$ are, a priori, "logarithmic packets" of degree $k$ at $\lambda_{0}=\frac{1}{2}$ and $\lambda_{0}=1$, respectively:

$$
\begin{aligned}
V_{\mathfrak{i}}^{k}(x, t) & =r_{\mathfrak{i}}^{1 / 2} \sum_{j=0}^{k} \frac{1}{j !}\left(\ln r_{\mathfrak{i}}\right)^{j} \mathcal{V}_{\mathfrak{i}}^{k j}\left(\varphi_{\mathfrak{i}}, t\right), \\
W_{\mathfrak{i}}^{k}(x, t) & =r_{\mathfrak{i}} \sum_{j=0}^{k} \frac{1}{j !}\left(\ln r_{\mathfrak{i}}\right)^{j} \mathcal{W}_{\mathfrak{i}}^{k j}\left(\varphi_{\mathfrak{i}}, t\right) .
\end{aligned}
$$

From the mere application of Proposition 5.2, it can be readily seen that the number of terms in the sums grows with $k \rightarrow \infty$, in general, and therefore, the abstract Theorem 2.1 cannot be employed in the framework of weighted spaces with separated asymptotics since these spaces must inflate from step to step of the iterative procedure.

Nevertheless, in the situation where the limit relaxation kernels $B^{\mathfrak{i}}$ do not depend on the angular variables $\varphi_{\mathfrak{i}}$, the degree of the singular parts is stationary at 0, as shown in Section 5.3, which restores the possibility of applying the abstract Theorem 2.1. Before investigating the most general situation, we first deal with this case. 


\subsection{Case of a smooth kernel: absence of logarithms}

We make the assumption (1.10) on the relaxation kernel $B$. Therefore we are in the situation where $\left\{P, Q^{ \pm}\right\}(t, \tau)$ has constant coefficients in $\varphi$ and the constructions of Section 5.3 apply. Let $\gamma$ such that $\ell-\gamma \in\left(1, \frac{3}{2}\right)$ and such that (6.1) holds.

We set $\beta=\gamma+1$, thus $\beta$ satisfies the condition prescribed in Theorem 3.5. We are going to use the abstract framework of Theorem 2.1 with the following choice for the spaces $\mathfrak{D}$ and $\mathfrak{R}$ : we choose subspaces of the couple $(\mathfrak{D}, \mathfrak{R})$ used before in $(3.13)$ in the form of spaces with detached asymptotics, where the asymptotics belong to the special classes $\mathfrak{U}\left(\frac{1}{2}\right)$ and $\mathfrak{H}\left(\frac{1}{2}\right)$ defined in (5.30)-(5.31) and (5.32), respectively;

$$
\begin{aligned}
\mathfrak{D}=\left\{u \in V_{\beta}^{\ell+1,0}(\Omega)^{2}: u\right. & \text { satisfies orthogonality condition }(2.8) \text { and } \\
u & \left.=\widetilde{w}+\sum_{\mathfrak{i}=\mathbf{I}, \mathbf{I I}} \chi\left(x_{\mathfrak{i}}\right) U_{\mathfrak{i}}\left(x_{\mathfrak{i}}\right), \quad \widetilde{w} \in V_{\gamma}^{\ell+1,1}(\Omega)^{2}, U_{\mathfrak{i}} \in \mathfrak{U}\left(\frac{1}{2}\right)\right\}, \\
\mathfrak{R}=\left\{\{f, g\} \in R_{\beta}^{\ell} V(\Omega): h\right. & :=\{f, g\} \text { satisfy compability condition }(2.7) \text { and } \\
h & \left.=\widetilde{h}+\sum_{\mathfrak{i}=\mathbf{I}, \mathbf{I I}} \chi\left(x_{\mathfrak{i}}\right) H_{\mathfrak{i}}\left(x_{\mathfrak{i}}\right), \quad \widetilde{h} \in R_{\gamma}^{\ell, 1} V(\Omega), H_{\mathfrak{i}} \in \mathfrak{H}\left(\frac{1}{2}\right)\right\},
\end{aligned}
$$

with the respective norms, $c f .(5.39)$ and (5.40),

$$
\begin{aligned}
\|u\|_{\mathfrak{D}} & =\left(\|\widetilde{w}\|_{V_{\gamma}^{\ell+1,1}(\Omega)}^{2}+\sum_{\mathfrak{i}=\mathbf{I}, \mathbf{I I}}\left\|U_{\mathfrak{i}}\right\|_{\mathfrak{U}(1 / 2)}^{2}\right)^{1 / 2} \\
\|h\|_{\mathfrak{R}} & =\left(\|\widetilde{h}\|_{R_{\gamma}^{\ell, 1} V(\Omega)}^{2}+\sum_{\mathfrak{i}=\mathbf{I}, \mathbf{I I}}\left\|H_{\mathfrak{i}}\right\|_{\mathfrak{H}(1 / 2)}^{2}\right)^{1 / 2} .
\end{aligned}
$$

Theorem 6.1. Let the assumption (1.10) on the relaxation kernel $B$ be satisfied. Let the right hand side of problem (1.13) satisfy for a $\gamma$ such that $\ell-\gamma \in\left(1, \frac{3}{2}\right)$

$$
\{f, g\} \in L_{\infty}\left(0, T ; R_{\gamma}^{\ell, 1} V(\Omega)\right), \quad \text { i.e., with } g^{-}\left(\mathcal{O}^{\mathfrak{i}}, t\right)=-g^{+}\left(\mathcal{O}^{\mathfrak{i}}, t\right),
$$

together with the compatibility condition (2.7). Let $\beta=\gamma+1$.

Then the solution $u \in L_{\infty}\left(0, T ; V_{\beta}^{\ell+1,0}(\Omega)^{2}\right)$ of problem (1.13) given by Theorem 3.5, admits the representation

$$
u(x, t)=\widetilde{w}(x, t)+\sum_{\mathfrak{i}=\mathbf{I}, \mathbf{I I}} \chi^{\mathfrak{i}}(x) r_{\mathfrak{i}}^{1 / 2} \mathcal{V}_{\mathbf{i}}\left(\varphi_{\mathfrak{i}}, t\right)
$$

where (recall that $\Upsilon$ denotes the interval $(-\pi, \pi)$ )

$$
\widetilde{w} \in L_{\infty}\left(0, T ; V_{\gamma}^{\ell+1,1}(\Omega)^{2}\right) \quad \text { and } \quad \mathcal{V}_{\mathbf{i}} \in L_{\infty}\left(0, T ; H^{\ell+1}(\Upsilon)^{2}\right),
$$

with the estimates

$$
\|\widetilde{u} ; t\|_{V_{\gamma}^{\ell+1}(\Omega)}+\left\|\mathcal{V}_{\mathbf{i}} ; t\right\|_{H^{\ell+1}(\Upsilon)} \leq c_{0} \mathrm{e}^{\delta_{0} t}\|\{f, g\} ; t\|_{R_{\gamma}^{\ell, 1} V(\Omega)} .
$$

Remark 6.2. In (6.10) and (6.11) the space $H^{\ell+1}(-\pi, \pi)$ has been mentioned only to fix ideas. Any Sobolev space $H^{m}$ could have been used either since, in the present situation, the angular functions are analytic on $[-\pi, \pi]$. 
Proof. We only have to prove that the assumptions of Theorem 2.1 are satisfied, namely that the operator $\left\{L(t), N^{ \pm}(t)\right\}$ is continuous and invertible from $\mathfrak{D}$ into $\mathfrak{R}$, with uniform norms in $t \in(0, T)$, and that $\left\{P(t, \tau), Q^{ \pm}(t, \tau)\right\}$ is continuous from $\mathfrak{D}$ into $\mathfrak{R}$, with uniform norms in $(t, \tau) \in \mathcal{T}(T)$.

The continuity from $\mathfrak{D}$ into $\mathfrak{R}$ relies on (5.41), on the continuity of $\left\{L(t), N^{ \pm}(t)\right\}$ and $\left\{P(t, \tau), Q^{ \pm}(t, \tau)\right\}$ from $V_{\gamma}^{\ell+1,1}(\Omega)^{2}$ into $R_{\gamma}^{\ell, 1} V(\Omega)$, and on the continuity of

$$
u \longmapsto \chi^{\mathfrak{i}}(x)\left(\left\{L\left(t, x, \nabla_{x}\right), N^{ \pm}\left(t, x, \nabla_{x}\right)\right\}-\left\{L\left(t, \mathcal{O}^{\mathfrak{i}}, \nabla_{x}\right), N^{ \pm}\left(t, \mathcal{O}^{\mathfrak{i}}, \nabla_{x}\right)\right\}\right) u
$$

from $\mathfrak{U}\left(\frac{1}{2}\right)$ into $R_{\gamma}^{\ell} V(\Omega)$, and similar for $\left\{P, Q^{ \pm}\right\}$.

The continuity of the inverse relies on (5.42) and on Theorem 4.10.

\subsection{Case of a sectorial kernel: accumulation of logarithms for iterate solutions}

The estimates given in the following proposition allow for the convergence proof of the double series resulting from (3.18) when using (6.2) combined with (6.3).

Proposition 6.3. We assume that the operators (1.11) and (1.12) satisfy conditions (1.6), (1.9) and (6.1). Let the right hand side of problem (1.13) satisfy for a $\gamma$ such that $\ell-\gamma \in\left(1, \frac{3}{2}\right)$ assumption (6.8) together with the compatibility condition (2.7). Let $u^{k}$ be the iterate solutions of instantaneous elasticity problems $\{L, N\} u^{k}=$ $\left\{f^{k}, g^{k}\right\}$ with right-hand sides (3.19) under orthogonality condition (2.8).

Then $u^{k}$ can be represented in the form (6.2), with the coefficients given by (6.3). In addition, the following estimates hold

$\left\|\widetilde{u}^{k} ; t\right\|_{V_{\gamma}^{\ell+1}(\Omega)}+\sum_{\mathfrak{i}=\mathbf{I}, \mathbf{I I}}\left(\left\|a_{\mathfrak{i}}^{k} ; t\right\|+\sum_{j=0}^{k} \delta_{1}^{j}\left[\left\|\nu_{\mathfrak{i}}^{k j} ; t\right\|_{H^{\ell+1}(\Upsilon)}+\left\|\mathcal{W}_{\mathfrak{i}}^{k j} ; t\right\|_{H^{\ell+1}(\Upsilon)}\right]\right) \leq \frac{c_{2}\left(\delta_{2} t\right)^{k}}{k !}\|\{f, g\} ; t\|_{R_{\gamma}^{\ell, 1} V(\Omega)}$,

where $\delta_{1}, \delta_{2}$ and $c_{2}$ are positive constants, independent of the integer $k$.

Proof. Let us denote by $\mathbf{N}(t)$ the norm

$$
\mathbf{N}(t):=\|\{f, g\} ; t\|_{R_{\gamma}^{\ell, 1} V(\Omega)} .
$$

Since $u^{0}$ solves the instantaneous elasticity problem (4.1), Theorem 4.10 ensures the representations (6.2) and (6.3) at $k=0$ together with formulae

$$
\begin{gathered}
\mathcal{V}_{\mathbf{i}}^{00}(\varphi, t)=b_{1, \mathfrak{i}}(t) \mathcal{V}_{1, \mathbf{i}}(\varphi, t)+b_{2, \mathfrak{i}}(t) \mathcal{V}_{2, \mathbf{i}}(\varphi, t) \\
\mathcal{W}_{\mathbf{i}}^{00}(\varphi, t)=c_{1, \mathbf{i}}(t) \mathcal{W}_{1, \mathbf{i}}(\varphi, t)+c_{2, \mathbf{i}}(t) \mathcal{W}_{2, \mathbf{i}}(\varphi, t)-x_{2} G_{\mathbf{i}}(t)
\end{gathered}
$$

and the estimate

$$
\left\|\widetilde{u}^{0} ; t\right\|_{V_{\gamma}^{\ell+1}(\Omega)}+\left\|a_{n, \mathbf{i}}^{0} ; t\right\|+\left\|b_{n, \mathbf{i}} ; t\right\|+\left\|c_{n, \mathbf{i}} ; t\right\|+\left\|G_{\mathbf{i}} ; t\right\| \leq C \mathbf{N}(t),
$$

where $G_{\mathfrak{i}}$ is determined according to Lemma 4.9 with $G(t)=g^{+}\left(\mathcal{O}^{\mathfrak{i}}, t\right)(c f .(6.8))$. Direct evaluation of norms of elements in (6.13) in the space $H^{\ell+1}(\Upsilon)$ shows that estimate (6.14) yields estimate (6.12) for $j=k=0$.

The proof is achieved by induction, thus we assume that (6.2), (6.3) and (6.12) hold for $k<K$ and we verify the same formulae for $k=K \geq 1$.

First of all, we observe, that owing to (4.22), assumption (6.8) provides inclusion (3.15) for any $\beta \in\left(\ell-\frac{1}{2}, l\right)$ while, in view of (6.1), we can choose the weight index $\beta$ such that

$$
\beta-\delta_{\beta}<\gamma \text { and } \beta-1<\gamma \text {. }
$$


Inequalities (6.15) allow to reduce the problem for $u^{K}$ in $\Omega$ to model problem (4.3). To this end, equations (4.1) for $u^{K}$ with the right-hand side (3.19) are multiplied by the cut-off function $\chi^{\mathfrak{i}}$ which leads to the problem

$$
\begin{gathered}
L^{\mathfrak{i}}\left(t, \nabla_{x}\right) \chi^{\mathfrak{i}}(x) u^{K}(x, t)=\chi^{\mathfrak{i}}(x)^{2} F_{\mathfrak{i}}^{K}(x, t)+\widetilde{F}_{\mathfrak{i}}^{K}(x, t), \quad x \in \mathbb{K}, \\
N^{\mathfrak{i} \pm}\left(t, \nabla_{x}\right) \chi^{\mathfrak{i}}(x) u^{K}(x, t)=\chi^{\mathfrak{i}}(x)^{2} G_{\mathfrak{i}}^{K \pm}(x, t)+\widetilde{G}_{\mathfrak{i}}^{K \pm}(x, t), \quad \varphi= \pm \pi,
\end{gathered}
$$

where

$$
\left\{F_{\mathfrak{i}}^{K}(x, t), G_{\mathfrak{i}}^{K \pm}(x, t)\right\}=-\int_{0}^{t}\left\{P^{\mathfrak{i}}\left(x, t, \tau, \nabla_{x}\right), Q^{\mathfrak{i} \pm}\left(x, t, \tau, \nabla_{x}\right)\right\}\left(V_{\mathfrak{i}}^{K-1}(x, \tau)+W_{\mathfrak{i}}^{K-1}(x, \tau)\right) \mathrm{d} \tau,
$$

and

$$
\begin{aligned}
& \left\{\widetilde{F}_{\mathfrak{i}}^{K}, \widetilde{G}_{\mathfrak{i}}^{K \pm}\right\}=\underbrace{\left[\left\{L^{\mathfrak{i}}, N^{\mathfrak{i} \pm}\right\}, \chi^{\mathfrak{i}}\right] u^{K}}_{I_{1}^{\mathfrak{i}}}-\underbrace{\chi^{\mathfrak{i}}\left\{L-L^{\mathfrak{i}}, N-N^{\mathfrak{i} \pm}\right\} u^{K}}_{I_{2}^{\mathfrak{i}}}
\end{aligned}
$$

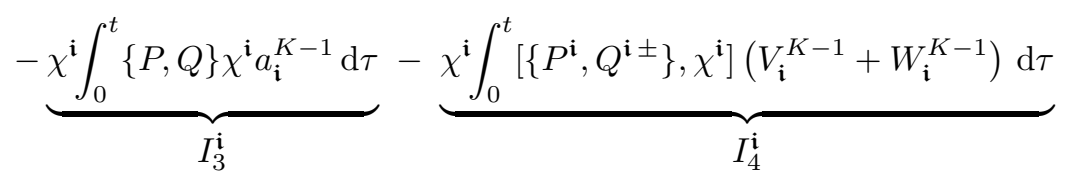

$$
\begin{aligned}
& -\underbrace{\chi^{\mathfrak{i}} \int_{0}^{t}\left[\left\{P-P^{\mathfrak{i}}, Q-Q^{\mathfrak{i} \pm}\right\}, \chi^{\mathfrak{i}}\right]\left(V_{\mathfrak{i}}^{K-1}+W_{\mathfrak{i}}^{K-1}\right) \mathrm{d} \tau}_{I_{5}^{\mathfrak{i}}}-\underbrace{\chi^{\mathfrak{i}} \int_{0}^{t}\{P, Q\} \widetilde{u}^{K-1} \mathrm{~d} \tau}_{I_{6}^{\mathbf{i}}} .
\end{aligned}
$$

The term $I_{1}^{\mathfrak{i}}$ includes the commutator $\left[\left\{L^{\mathfrak{i}}, N^{\mathfrak{i} \pm}\right\}, \chi^{\mathfrak{i}}\right]$ which vanishes near the tip $\mathcal{O}^{\mathfrak{i}}$, hence

$$
\left\|I_{1}^{\mathrm{i}} ; t\right\|_{R_{\varkappa}^{\ell} V(\mathbb{K})} \leq c\left\|u^{K} ; t\right\|_{V_{\beta}^{\ell+1,0}(\Omega)}
$$

with any $\varkappa \in \mathbb{R}$, in particular for $\varkappa=\gamma$. The presence of the right factor $D\left(\nabla_{x}\right)$ in (5.1) implies that the point $\mathcal{O}^{\mathfrak{i}}$ is outside of the support of $I_{4}^{\mathfrak{i}}$, so the induction insures that

$$
\left\|I_{3}^{\mathfrak{i}} ; t\right\|_{R_{\gamma}^{\ell} V(\Omega)} \leq c \int_{0}^{t}\left|a_{\mathfrak{i}}^{K-1}(\tau)\right| \mathrm{d} \tau \leq c \frac{c_{2}}{\delta_{2}} \frac{1}{K !}\left(\delta_{2} t\right)^{K} \mathbf{N}(t) .
$$

Next, an application of estimate (6.12) for the remainder $\widetilde{u}^{K}$ leads to

$$
\begin{aligned}
\left\|I_{6}^{\mathrm{i}} ; t\right\|_{R_{\gamma}^{\ell} V(\mathbb{K})} & \leq c \int_{0}^{t}\left\|\widetilde{u}^{K-1} ; \tau\right\|_{V_{\gamma}^{\ell+1,0}(\Omega)} \mathrm{d} \tau \\
& \leq c c_{2} \int_{0}^{t} \frac{1}{(K-1) !}\left(\delta_{2} \tau\right)^{K-1} \mathbf{N}(\tau) \mathrm{d} \tau \leq c c_{2} \frac{1}{K !}\left(\delta_{2} t\right)^{K} \mathbf{N}(t) .
\end{aligned}
$$

Furthermore, by (6.15) and the following inequality

$$
\left|\nabla_{x}^{j}\left(A(x, t)-A\left(\mathcal{O}^{\mathfrak{i}}, t\right)\right)\right| \leq c_{A j}^{\mathfrak{i}} r_{\mathfrak{i}}^{\delta_{j}, 0} \leq C_{A j}^{\mathfrak{i}} r_{\mathfrak{i}}^{1-j},
$$

we find that, by virtue of (4.4), (5.3) and (3.20),

$$
\begin{aligned}
\left\|I_{2}^{\mathrm{i}} ; t\right\|_{R_{\gamma}^{\ell} V(\mathbb{K})} & \leq c\left\|r_{\mathfrak{i}} \nabla_{x} u^{K} ; t\right\|_{V_{\gamma}^{\ell}\left(\Omega \cap \operatorname{supp} \chi^{\mathrm{i}}\right)} \\
& \leq c\left\|u^{K} ; t\right\|_{V_{\gamma+1}^{\ell+1,0}(\Omega)} \leq c\left\|u^{K} ; t\right\|_{V_{\beta}^{\ell+1,0}(\Omega)} \leq c \frac{c_{0}}{\delta_{0}} \frac{1}{K !}\left(\delta_{0} t\right)^{K} \mathbf{N}(t) .
\end{aligned}
$$


By an application of Lemma 5.1, in view of estimate (6.12) with $k=K-1$, to the angular parts $\mathcal{V}^{K-1 \mathfrak{i}}$ and $\mathcal{W}^{K-1 \mathrm{i}}$ it follows that

$$
\begin{aligned}
& \left\|V_{\mathbf{i}}^{K-1} ; t\right\|_{V_{\beta}^{\ell+1}\left(\mathbb{K}_{1}\right)}+\left\|W_{\mathbf{i}}^{K-1} ; t\right\|_{V_{\beta}^{\ell+1}\left(\mathbb{K}_{1}\right)} \\
& \leq s \sum_{j=0}^{K-1} d^{j}\left(\frac{1}{\left(\beta-l+\frac{1}{2}\right)^{j+1}}\left\|\mathcal{V}_{\mathbf{i}}^{K-1} ; t\right\|_{H^{\ell+1}(\Upsilon)}+\frac{1}{(\beta-l+1)^{j+1}}\left\|\mathcal{W}_{\mathbf{i}}^{K-1} ; t\right\|_{H^{\ell+1}(\Upsilon)}\right) \\
& \quad \leq c c_{2} \frac{2}{(K-1) !}\left(\delta_{2} t\right)^{K-1} \mathbf{N}(t)\left\{\sum_{j=0}^{K-1} \frac{\delta_{1}^{-j} d^{j}}{\left(\beta-l+\frac{1}{2}\right)^{j+1}}\right\}
\end{aligned}
$$

For $\delta_{1}>0$ sufficiently large, the sum in curly brackets in (6.24) is bounded by the constant

$$
\mathbf{C}=\left(\beta-l+\frac{1}{2}\right)^{-1}\left(1-d\left[\delta_{1}\left(\beta-l+\frac{1}{2}\right)\right]^{-1}\right)^{-1},
$$

since $(\beta-l+1)>\left(\beta-l+\frac{1}{2}\right)>0$ by the restriction required in Theorem 3.3. The derived estimate serves for processing the terms $I_{4}^{\mathrm{i}}$ and $I_{5}^{\mathrm{i}}$ in the same way as with $I_{1}^{\mathrm{i}}$ and $I_{2}^{\mathrm{i}}$ :

$$
\left\|I_{4}^{\mathrm{i}} ; t\right\|_{R_{\varkappa}^{\ell} V(\mathbb{K})} \leq c \int_{0}^{t}\left(\left\|V_{\mathbf{i}}^{K-1} ; \tau\right\|_{V_{\beta}^{\ell+1}\left(\mathbb{K}_{1}\right)}+\left\|W_{\mathbf{i}}^{K-1} ; \tau\right\|_{V_{\beta}^{\ell+1}\left(\mathbb{K}_{1}\right)}\right) \mathrm{d} \tau \leq c \mathbf{C} \frac{1}{K !}\left(\delta_{2} t\right)^{K} \mathbf{N}(t),
$$

for all $\varkappa \in \mathbb{R}$, and

$$
\begin{array}{r}
\left\|I_{5}^{\mathfrak{i}} ; t\right\|_{R_{\gamma}^{\ell} V(\mathbb{K})} \leq c \int_{0}^{t}\left(\left\|r_{\mathfrak{i}}^{\delta_{B}} V_{\mathfrak{i}}^{K-1} ; \tau\right\|_{V_{\gamma}^{\ell+1}\left(\mathbb{K}_{1}\right)}+\left\|r_{\mathfrak{i}}^{\delta_{B}} W_{\mathfrak{i}}^{K-1} ; \tau\right\|_{V_{\gamma}^{\ell+1}\left(\mathbb{K}_{1}\right)}\right) \mathrm{d} \tau \\
\leq c \int_{0}^{t}\left(\left\|V_{\mathfrak{i}}^{K-1} ; \tau\right\|_{V_{\beta}^{\ell+1}\left(\mathbb{K}_{1}\right)}+\left\|W_{\mathfrak{i}}^{K-1} ; \tau\right\|_{V_{\beta}^{\ell+1}\left(\mathbb{K}_{1}\right)}\right) \mathrm{d} \tau \\
\leq c \mathbf{C} \frac{c_{2}}{\delta_{2}} \frac{1}{K !}\left(\delta_{2} t\right)^{K} \mathbf{N}(t) .
\end{array}
$$

Stabilization condition (1.9) and the first inequality in (6.15) are applied in (6.26).

It is supposed that $\delta_{2} \geq \delta_{0}$, where $\delta_{0}$ denotes the constant in Theorem 3.5. Thus, the norms indicated in (6.19), (6.23), (6.20), (6.25), (6.26), (6.21) are bounded by the common majorant

$$
c\left(\frac{1}{\delta_{0}}+\frac{1}{\delta_{2}}\right) \frac{1}{K !}\left(\delta_{2} t\right)^{K} \mathbf{N}(t)
$$

Using representations (6.17), (6.3), estimates (6.12) with $k=K-1$ and Proposition 5.4, we conclude that

$$
\begin{aligned}
\left\{F_{\mathfrak{i}}^{K}(x, t), G_{\mathfrak{i}}^{K}(x, t)\right\}=-\sum_{j=0}^{K-1} \frac{1}{j !} & \left(\ln r_{\mathfrak{i}}\right)^{j} \\
& \times \int_{0}^{t}\left(\left\{r^{-3 / 2} \mathcal{F}_{\mathfrak{i}}^{K j}\left(\varphi_{\mathfrak{i}}, \tau\right), r^{-1 / 2} \mathcal{G}_{\mathfrak{i}}^{K j \pm}(\tau)\right\}+\left\{r^{-1} \mathcal{H}_{\mathfrak{i}}^{K j}\left(\varphi_{\mathfrak{i}}, \tau\right), r^{0} \mathcal{K}_{\mathfrak{i}}^{K j \pm}(\tau)\right\}\right) \mathrm{d} \tau,
\end{aligned}
$$

with the estimates

$$
\left\|\left\{\mathcal{F}_{\mathbf{i}}^{K j}, \mathcal{G}_{\mathbf{i}}^{K j \pm}\right\} ; \tau\right\|_{R^{\ell} H(\Upsilon)}+\left\|\left\{\mathcal{H}_{\mathbf{i}}^{K j}, \mathcal{K}_{\mathfrak{i}}^{K j \pm}\right\} ; \tau\right\|_{R^{\ell} H(\Upsilon)} \leq c \delta_{1}^{-j} \frac{\left(\delta_{2} \tau\right)^{K-1}}{(K-1) !} \mathbf{N}(\tau)
$$


Thus, by an application of Proposition 5.2 for three values $\lambda_{0}=0, \lambda_{0}=\frac{1}{2}$ and $\lambda_{0}=1$, and Theorem 4.2, the following decomposition of the solution to model problem (6.16) is obtained

$$
\chi^{\mathfrak{i}}(x) u^{K}(x, t)=a_{\mathfrak{i}}^{K}(t)+V_{\mathfrak{i}}^{K}(x, t)+W_{\mathfrak{i}}^{K}(x, t)+\widetilde{u}_{\mathfrak{i}}^{K}(x, t),
$$

where $V_{\mathbf{i}}^{K}$ and $W_{\mathbf{i}}^{K}$ take the form (6.3) and, moreover,

$$
\begin{aligned}
\left\|\widetilde{u}_{\mathfrak{i}}^{K} ; t\right\|_{R_{\gamma}^{\ell} V(\mathbb{K})}+\left\|a_{\mathfrak{i}}^{K} ; t\right\| & +\sum_{j=0}^{K} \delta_{1}^{j}\left(\left\|\mathcal{V}_{\mathfrak{i}}^{K j} ; t\right\|_{H^{\ell+1}(\Upsilon)}+\left\|\mathcal{W}_{\mathfrak{i}}^{K j} ; t\right\|_{H^{\ell+1}(\Upsilon)}\right) \\
& \leq c\left\{\frac{1}{\delta_{0}} \frac{1}{K !}\left(\delta_{2} t\right)^{K}+\int_{0}^{t} \frac{1}{(K-1) !}\left(\delta_{2} \tau\right)^{K-1} d \tau\right\} \mathbf{N}(t) \\
& \leq c\left(\frac{1}{\delta_{0}}+\frac{1}{\delta_{1}}\right) \frac{1}{K !}\left(\delta_{2} t\right)^{K} \mathbf{N}(t) .
\end{aligned}
$$

In order to derive the asymptotic formula for $u^{K}$ from (6.29) we proceed as follows. (6.29) is multiplied by the cut-off function $\chi^{\mathfrak{i}}$, and the contributions for $\mathfrak{i}=\mathbf{I}, \mathbf{I I}$ and the term

$$
\left\{1-\left(\chi^{\mathbf{I}}\right)^{2}-\left(\chi^{\mathbf{I I}}\right)^{2}\right\} u^{K}
$$

are assembled together. The estimate

$$
\left\|\left\{1-\left(\chi^{\mathbf{I}}\right)^{2}-\left(\chi^{\mathbf{I I}}\right)^{2}\right\} u^{K} ; t\right\|_{V_{\gamma}^{\ell+1}(\Omega)} \leq\left\|u^{K} ; t\right\|_{V_{\beta}^{\ell+1,0}(\Omega)} \leq \frac{c}{\delta_{0}} \frac{1}{K !}\left(\delta_{0} t\right)^{K} \mathbf{N}(t)
$$

is obtained, taking into account that $\chi^{\mathbf{I}}+\chi^{\mathbf{I I}}=1$ near the points $\mathcal{O}^{\mathbf{i}}$.

Thus, we have proved representation (6.2), (6.3) and estimate (6.12) for $k=K$. Since the majorant in (6.12) is of the form (6.27) with the constant $c$ which now should be selected in the appropriate way. It is possible, for the numbers $\delta_{0}$ and $\delta_{2} \geq \delta_{0}$ chosen in such a way that the majorant in (6.12) is smaller than $c_{2}(K !)^{-1}\left(\delta_{2} t\right)^{K} \mathbf{N}(t)$.

\subsection{Case of a sectorial kernel: theorem on asymptotics with logarithmic packets}

All preparations for our final result on the asymptotics of solutions of the creep problem are now complete and we are ready to prove:

Theorem 6.4. Let $\{f, g\}$ satisfy (6.8), where the smoothness index $l$ and the weight index $\gamma$ are related by (4.22). Let $\beta=\gamma+1$. Then the solution $u \in L_{\infty}\left(0, T ; V_{\beta}^{\ell+1,0}(\Omega)^{2}\right)$ of the creep problem (1.13), given by Theorem 3.5, admits the representation

$$
u(x, t)=\widetilde{u}(x, t)+\sum_{\mathbf{i}} \chi^{\mathfrak{i}}(x)\left(a_{\mathfrak{i}}(t)+r_{\mathbf{i}}^{1 / 2} \mathcal{V}_{\mathbf{i}}\left(\ln r_{\mathfrak{i}}, \varphi_{\mathbf{i}}, t\right)+r_{\mathfrak{i}} \mathcal{W}_{\mathfrak{i}}\left(\ln r_{\mathfrak{i}}, \varphi_{\mathbf{i}}, t\right)\right),
$$

where

and (recall that $\Upsilon$ denotes the interval $(-\pi, \pi)$ )

$$
\widetilde{u} \in L_{\infty}\left(0, T ; V_{\gamma}^{\ell+1}(\Omega)^{2}\right), \quad a_{\mathfrak{i}} \in L_{\infty}(0, T)^{2},
$$

$$
\begin{aligned}
& \mathcal{V}_{\mathbf{i}}(\ln r, \varphi, t)=\sum_{j=0}^{\infty} \frac{1}{j !}(\ln r)^{j} \mathcal{V}_{\mathbf{i}}^{j}(\varphi, t), \quad \mathcal{V}_{\mathfrak{i}}^{j} \in L_{\infty}\left(0, T ; H^{\ell+1}(\Upsilon)^{2}\right) \\
& \mathcal{W}_{\mathfrak{i}}(\ln r, \varphi, t)=\sum_{j=0}^{\infty} \frac{1}{j !}(\ln r)^{j} \mathcal{W}_{\mathfrak{i}}^{j}(\varphi, t), \mathcal{W}_{\mathfrak{i}}^{j} \in L_{\infty}\left(0, T ; H^{\ell+1}(\Upsilon)^{2}\right)
\end{aligned}
$$


with the estimates, for positive constants $\delta_{1}, \delta_{2}$ and $c_{2}$ :

$$
\begin{gathered}
\|\widetilde{u} ; t\|_{V_{\gamma}^{\ell+1}(\Omega)}+\left\|a_{\mathfrak{i}} ; t\right\| \leq c_{2} \mathrm{e}^{\delta_{2} t}\|\{f, g\} ; t\|_{R_{\gamma}^{\ell, 1} V(\Omega)} \\
\left\|\mathcal{V}_{\mathfrak{i}}^{j} ; t\right\|_{H^{\ell+1}(\Upsilon)}+\left\|\mathcal{W}_{\mathfrak{i}}^{j} ; t\right\|_{H^{\ell+1}(\Upsilon)} \leq c_{2} \frac{1}{j !}\left(\frac{\delta_{2}}{\delta_{1}} t\right)^{j} \mathrm{e}^{\delta_{2} t}\|\{f, g\} ; t\|_{R_{\gamma}^{\ell, 1} V(\Omega)} .
\end{gathered}
$$

Moreover, the logarithmic packets (6.32) verify the estimate

$$
\left\|\mathcal{V}_{\mathbf{i}}(\ln r, \cdot, \cdot) ; t\right\|_{H^{\ell+1}(\Upsilon)}+\left\|\mathcal{W}_{\mathbf{i}}(\ln r, \cdot, \cdot) ; t\right\|_{H^{\ell+1}(\Upsilon)} \leq c_{2} \mathrm{e}^{\delta_{2} t} \cosh \left(2 \sqrt{\frac{\delta_{2}}{\delta_{1}} t|\ln r|}\right)\|\{f, g\} ; t\|_{R_{\gamma}^{\ell, 1} V(\Omega)} .
$$

Proof. We set

$$
a^{\mathfrak{i}}=\sum_{k=0}^{\infty} a_{\mathfrak{i}}^{k}, \quad \widetilde{u}=\sum_{k=0}^{\infty} \widetilde{u}^{k}
$$

an application of estimates (6.12) yields inclusions (6.31) and estimate (6.33). Owing to (6.3) we have

$$
\mathcal{V}_{\mathbf{i}}^{j}=\sum_{k=j}^{\infty} \mathcal{V}_{\mathfrak{i}}^{k j}, \quad \mathcal{W}_{\mathbf{i}}^{j}=\sum_{k=j}^{\infty} \mathcal{W}_{\mathfrak{i}}^{k j}
$$

Estimates (6.12) combined with the inequality $m ! n ! \leq(m+n)$ ! leads to

$$
\left\|\mathcal{V}_{\mathbf{i}}^{j} ; t\right\|_{H^{\ell+1}(\Upsilon)} \leq c_{2} \delta_{1}^{-j} \sum_{k=j}^{\infty} \frac{1}{k !}\left(\delta_{2} t\right)^{k} \mathbf{N}(t) \leq c_{2} \frac{1}{j !}\left(\frac{\delta_{2}}{\delta_{1}} t\right)^{j} \mathbf{N}(t) \sum_{n=0}^{\infty} \frac{1}{n !}\left(\delta_{2} t\right)^{n} .
$$

The same evaluation performed for $\mathcal{W}_{\mathfrak{i}}^{j}$ results in (6.34). To show (6.35), we need the inequality

$$
2^{2 j} \frac{(j !)^{2}}{(2 j) !}=\frac{2 \cdot 4 \cdot \ldots \cdot 2 j}{1 \cdot 3 \cdot \ldots \cdot(2 j-1)} \cdot \frac{2 \cdot 4 \cdot \ldots \cdot 2 j}{2 \cdot 4 \cdot \ldots \cdot 2 j}>1
$$

and then we obtain

$$
\begin{aligned}
\left\|\mathcal{V}_{\mathbf{i}} ; t\right\|_{H^{\ell+1}(\Upsilon)} & \leq c_{2} \exp \left(\delta_{2} t\right) \sum_{j=0}^{\infty} \frac{2^{2 j}}{(2 j) !}\left(\frac{\delta_{2}}{\delta_{1}} t|\ln r|\right)^{j} \mathbf{N}(t) \\
& =c_{2} \exp \left(\delta_{2} t\right) \cosh \left(2 \sqrt{\frac{\delta_{2}}{\delta_{1}} t|\ln r|}\right) \mathbf{N}(t)
\end{aligned}
$$

The terms $\mathcal{W}_{\mathbf{i}}$ are estimated in the same way, which ends the proof.

Remark 6.5. If the data $\{f, g\}$ belong to $L_{\infty}\left(0, T ; R_{\gamma}^{\ell} H(\Omega)\right)$ without fulfilling (6.8), the compatibility conditions (4.27) at the crack tips $\mathcal{O}^{i}$ are violated. Therefore a logarithmic term appears in the decomposition (6.2) of the solution $u^{0}$, see (4.37). Each step of the iterative procedure brings a new term $\ln r_{\mathfrak{i}}$. Thus, finally, the summation over $j=0, \ldots, k$ in $(6.3)$ for $W_{\mathfrak{i}}^{k}$ must be replaced by a summation over $j=0, \ldots, k+1$. 
However estimate (6.12) is still valid (possibly, with new constants $c_{2}$ and $\delta_{1}, \delta_{2}$ ). Moreover, Theorem 6.4 applies, provided we perform the replacement

$$
c_{2} \mapsto c_{2}\left(1+\delta_{1}^{-1}\right)
$$

in (6.33)-(6.35). Indeed, in view of the modification of summation proposed above for (6.3), formula (6.36) is now

$$
\mathcal{V}_{\mathbf{i}}^{j}=\sum_{k=j}^{\infty} \mathcal{V}_{\mathfrak{i}}^{k j}, \quad \mathcal{W}_{\mathfrak{i}}^{j}=\sum_{k=(j-1)_{+}}^{\infty} \mathcal{W}_{\mathfrak{i}}^{k j}
$$

It can be readily seen that inequality (6.34) remains valid under the replacement (6.37).

Remark 6.6. The stresses induced by the displacement field (6.30) have an asymptotic representation containing logarithmic packets at the exponents $-\frac{1}{2}$ and 0 :

$$
\sigma(u ; x, t)=\widetilde{\sigma}(x, t)+\sum_{\mathfrak{i}=\mathbf{I}, \mathbf{I I}} \chi^{\mathfrak{i}}(x)\left[r_{\mathfrak{i}}^{-1 / 2} \mathcal{S}_{\mathfrak{i}}\left(\ln r_{\mathfrak{i}}, \varphi_{\mathfrak{i}}, t\right)+\mathcal{T}_{\mathfrak{i}}\left(\ln r_{\mathfrak{i}}, \varphi_{\mathbf{i}}, t\right)\right]
$$

with $\widetilde{\sigma} \in L_{\infty}\left(0, T ; V_{\gamma}^{\ell}(\Omega)^{4}\right)$ and the estimates

$$
\begin{gathered}
\|\widetilde{\sigma} ; t\|_{V_{\gamma}^{\ell}(\Omega)} \leq C_{2} \mathrm{e}^{\delta_{2} t}\|\{f, g\} ; t\|_{R_{\gamma}^{\ell, 1} V(\Omega)}, \\
\left\|\mathcal{S}_{\mathfrak{i}} ; t\right\|_{H^{\ell}(\Upsilon)}+\left\|\mathcal{T}_{\mathfrak{i}} ; t\right\|_{H^{\ell}(\Upsilon)} \leq C_{2} \mathrm{e}^{\delta_{2} t} \cosh \left(2 \sqrt{\frac{\delta_{2}}{\delta_{1}} t|\ln r|}\right)\|\{f, g\} ; t\|_{R_{\gamma}^{\ell, 1} V(\Omega)} .
\end{gathered}
$$

Since $\ell \geq 2$ and $\ell-\gamma \in\left(1, \frac{3}{2}\right)$, the Sobolev embeddings and Lemma 3.2 allow to deduce from (6.39) pointwise estimates on $\Omega$ for $\widetilde{\sigma}$ and on any ring $0<r_{a} \leq r_{\mathfrak{i}} \leq r_{b}$ for $\mathcal{S}_{\mathfrak{i}}$ and $\mathcal{T}_{\mathfrak{i}}$.

\section{Further REMARKS ON THE LOGARITHMiC PACKETS}

In this section we give sufficient conditions for the presence of logarithmic packets in asymptotic expansions of elastic fields near the crack tips. We assume, moreover, the following independence on time of the Hooke matrix $A$ and the relaxation kernel $B$ :

$$
A(x, t) \equiv A(x) \quad \text { and } \quad B(x, t, \tau) \equiv B(x) .
$$

\subsection{Sufficient conditions for the presence of logarithms}

Let us suppose as before that $\{f, g\}$ belongs to $L_{\infty}\left(0, T ; R_{\gamma}^{\ell, 1} V(\Omega)\right)$.

As a first insight into the possible appearance of logarithms, let us come back to the starting steps of the construction of the series $\sum_{k} u^{k}$ in (3.18). Expansion (4.35) is valid for $u^{0}$, which now take the form, since the angular parts $\mathcal{V}_{j}$ are constant in $t$,

$$
u^{0}(x)=\widetilde{w}^{0}(x)+\sum_{\mathfrak{i}=\mathbf{I}, \mathbf{I I}} \chi^{\mathfrak{i}}(x) r_{\mathfrak{i}}^{1 / 2} \sum_{n=1,2} b_{n, \mathbf{i}}(t) \mathcal{V}_{n, \mathbf{i}}\left(\varphi_{\mathbf{i}}\right) \quad \text { with } \quad \widetilde{w}^{0} \in V_{\gamma}^{\ell+1,1}(\Omega)^{2} .
$$

Therefore, in view of (3.19), the right hand side $\left\{f^{1}, g^{1}\right\}$ for $u^{1}$ can be split according to

$$
\begin{aligned}
& f^{1}(x, t)=\widetilde{f}^{1}(x, t)-\sum_{\mathfrak{i}=\mathbf{I}, \mathbf{I I}} \chi^{\mathfrak{i}}(x) r_{\mathfrak{i}}^{-3 / 2} \sum_{n=1,2} \bar{b}_{n, \mathbf{i}}(t) \mathcal{P}^{\mathfrak{i}}\left(\varphi, \partial_{\varphi}, \frac{1}{2}\right) \mathcal{V}_{n, \mathbf{i}}(\varphi), \\
& g^{1}(x, t)=\widetilde{g}^{1}(x, t)-\sum_{\mathfrak{i}=\mathbf{I}, \mathbf{I I}} \chi^{\mathfrak{i}}(x) r_{\mathfrak{i}}^{-1 / 2} \sum_{n=1,2} \bar{b}_{n, \mathfrak{i}}(t) \mathcal{Q}^{\mathfrak{i} \pm}\left(\partial_{\varphi_{\mathfrak{i}}}, \frac{1}{2}\right) \mathcal{V}_{n, \mathbf{i}}( \pm \pi),
\end{aligned}
$$


where $\left\{\widetilde{f}^{1}, \widetilde{g}^{1}\right\} \in L_{\infty}\left(0, T ; R_{\gamma}^{\ell} H(\Omega)\right)$, see (4.33), and

$$
\bar{b}_{n, \mathbf{i}}(t)=\int_{0}^{t} b_{n, \mathbf{i}}(\tau) \mathrm{d} \tau .
$$

We further consider one of the crack tips and omit the subscript $\mathfrak{i}=\mathbf{I}$, II.

Since $u^{1}(x, t)$ is a solution of the instantaneous elasticity problem (4.1) with right-hand side $\left\{f^{1}, g^{1}\right\}$, according to Lemma 4.4 (iii) the asymptotic decomposition (6.2) of $u^{1}$ does not contain logarithms associated with the exponent $\frac{1}{2}$ (i.e., $\mathcal{V}_{\mathbf{i}}^{11}=0$ in (6.3)) if and only if the orthogonality condition (4.17) is satisfied with

$$
\mathcal{F}=\sum_{n=1,2} \bar{b}_{n}(t) \mathcal{P}\left(\varphi, \partial_{\varphi}, \frac{1}{2}\right) \mathcal{V}_{n}(\varphi) \quad \text { and } \quad \mathcal{G}^{ \pm}=\sum_{n=1,2} \bar{b}_{n}(t) \mathcal{Q}^{ \pm}\left(\partial_{\varphi_{\mathbf{i}}}, \frac{1}{2}\right) \mathcal{V}_{n}( \pm \pi)
$$

Condition (4.17) will be satisfied for any right hand side $\{f, g\}$, i.e., for any coefficient (7.3) if and only if

$$
\mathscr{Y}\left(\mathcal{V}_{j}^{*} \mid \mathcal{V}_{n}\right)=0 \text { for } n, j=1,2 \text {, }
$$

with

$$
\mathscr{Y}\left(\mathcal{V}_{j}^{*} \mid \mathcal{V}_{n}\right):=\int_{-\pi}^{\pi} \mathcal{V}_{j}^{*}(\varphi)^{\top} \mathcal{P}\left(\varphi, \partial_{\varphi}, \frac{1}{2}\right) \mathcal{V}_{n}(\varphi) \mathrm{d} \varphi+\sum_{ \pm} \mathcal{V}_{j}^{*}( \pm \pi)^{\top} \mathcal{Q}^{ \pm}\left(\partial_{\varphi}, \frac{1}{2}\right) \mathcal{V}_{n}( \pm \pi)
$$

We set, as usual,

$$
V_{n}(x)=r^{1 / 2} \mathcal{V}_{n}(\varphi), \quad V_{j}^{*}(x)=r^{-1 / 2} \mathcal{V}_{j}^{*}(\varphi)
$$

Multiplying (7.4) with $r^{-1} \mathrm{~d} r=r^{-1 / 2} r^{-3 / 2} r \mathrm{~d} r$, integrating in $r \in(1,2)$, with the change to the Cartesian coordinates in the annulus $\Xi=\{x: r \in(1,2),|\varphi|<\pi\}$, yields

$$
\begin{aligned}
(\ln 2) \mathscr{Y}\left(\mathcal{V}_{j}^{*} \mid \mathcal{V}_{n}\right)= & \int_{\Xi} V_{j}^{*}(x)^{\top} D\left(-\nabla_{x}\right)^{\top} B^{\mathfrak{i}}(\varphi) D\left(\nabla_{x}\right) V_{n}(x) \mathrm{d} x \\
& +\sum_{ \pm} \int_{1}^{2} V_{j}^{*}\left(x_{1}, \pm 0\right)^{\top} D\left(\mp e_{2}\right)^{\top} B^{\mathfrak{i}}(\varphi) D\left(\nabla_{x}\right) V_{n}\left(x_{1}, \pm 0\right) \mathrm{d} x_{1} \\
= & \left(B^{\mathfrak{i}} D\left(\nabla_{x}\right) V_{n}, D\left(\nabla_{x}\right) V_{j}^{*}\right)_{\Xi}=: \mathfrak{M}_{n j} .
\end{aligned}
$$

Here $B^{\mathfrak{i}}(\varphi)$ is the limit (1.9) of the kernel $B, c f .(5.2)$. Note that integrals over the circles $\partial \mathbb{B}_{1}$ and $\partial \mathbb{B}_{2}$, which appear when using Green's formula, cancel each other, since their common integrand is a homogeneous function of $r$ of order -1 .

Let $\mathfrak{M}$ be the $2 \times 2$ matrix with coefficients $\mathfrak{M}_{n j}$.

Strain columns $D\left(\nabla_{x}\right) V_{n}$ for $n=1,2$ are linearly independent in $\Xi$. So are $D\left(\nabla_{x}\right) V_{j}^{*}$ for $j=1,2$. Therefore, one can find a matrix $B^{\mathfrak{i}}(\varphi)$ such that $\mathfrak{M}$ is non-singular ${ }^{2}$. In such a case the conditions defined by (7.4) are not satisfied in general, and the term $u^{1}$ gets logarithms in its asymptotic form (6.2). In addition, from the theory of weight functions $(c f .[20,25])$ it follows that the stress intensity factors $b_{j}(t)$ in $(6.13)$ can attain any value by the appropriate selection of the load $\left.g\right|_{\partial \Omega}$.

In the following assertion we prove that the same condition det $\mathfrak{M} \neq 0$ produces infinite logarithmic packets (or no singularity at all) in the asymptotic decomposition (6.30) of elastic fields in viscoelastic anisotropic bodies with special cylindrical inhomogeneity of their relaxation kernel.

\footnotetext{
2 The results in Section 5.3 imply that $\mathfrak{M} \equiv 0$ for any matrix $B^{\mathfrak{i}}$ independent of $\varphi$. A non-trivial dependence in $\varphi$ is necessary to obtain that $\mathfrak{M}$ is non-singular.
} 
Theorem 7.1. Let the matrices $A$ and $B$ be independent of time variables $t, \tau$, and the matrix $\mathfrak{M}$ with entries (7.6) be non-singular. If the function $\ln r \rightarrow \mathcal{V}(\ln r, \varphi, t)$ in (6.32) is a polynomial and the corresponding sum is finite, then $\mathcal{V}(\ln r, \varphi, t)=0$.

Proof. We suppose that

$$
\mathcal{V}(\ln r, \varphi, t)=\sum_{j=0}^{k} \frac{1}{j !}(\ln r)^{j} \mathcal{V}^{j}(\varphi, t),
$$

and the coefficient $\mathcal{V}^{k} \not \equiv 0$. We put the corresponding asymptotic expansion (6.30) into problem (1.2). Using formulas (4.4) and (5.3), and assembling together the coefficients of the expression $r^{-3 / 2} \frac{1}{k !}(\ln r)^{k}$ in the interior equation and of the term $r^{-1 / 2} \frac{1}{k !}(\ln r)^{k}$ in the boundary condition of (1.2), the following identity is derived

$$
\begin{aligned}
& \mathcal{L}\left(\varphi, \partial_{\varphi}, \frac{1}{2}\right) \mathcal{V}^{k}(\varphi, t)=-\int_{0}^{t} \mathcal{P}\left(\varphi, \partial_{\varphi}, \frac{1}{2}\right) \mathcal{V}^{k}(\varphi, \tau) \mathrm{d} \tau, \varphi \in \Upsilon, \\
& \mathcal{N}\left(\partial_{\varphi}, \frac{1}{2}\right) \mathcal{V}^{k}( \pm \pi, t)=-\int_{0}^{t} \mathcal{Q}^{ \pm}\left(\partial_{\varphi}, \frac{1}{2}\right) \mathcal{V}^{k}( \pm \pi, \tau) \mathrm{d} \tau .
\end{aligned}
$$

We here used the formula $r \partial_{r} r^{1 / 2}(\ln r)^{j}=r^{1 / 2}(\ln r)^{j}\left\{1+j(\ln r)^{-1}\right\}$ which shows that we do not need to pay attention to the differentiation of logarithms, so that the terms of lower order in (7.7) can be neglected.

From compatibility conditions (4.17) for problem (4.16) it follows that for a.e. $t \in(0, T)$

$$
\int_{0}^{t}\left\{\int_{-\pi}^{\pi} \mathcal{V}_{j}^{*}(\varphi)^{\top} \mathcal{P}\left(\varphi, \partial_{\varphi}, \frac{1}{2}\right) \mathcal{V}^{k}(\varphi, \tau) \mathrm{d} \varphi+\sum_{ \pm} \mathcal{V}_{j}^{*}( \pm \pi)^{\top} \mathcal{Q}^{ \pm}\left(\partial_{\varphi}, \frac{1}{2}\right) \mathcal{V}^{k}( \pm \pi, \tau)\right\} \mathrm{d} \tau=0
$$

for $j=1,2$, thus

$$
\int_{-\pi}^{\pi} \mathcal{V}_{j}^{*}(\varphi)^{\top} \mathcal{P}\left(\varphi, \partial_{\varphi}, \frac{1}{2}\right) \mathcal{V}^{k}(\varphi, t) \mathrm{d} \varphi+\sum_{ \pm} \mathcal{V}_{j}^{*}( \pm \pi)^{\top} \mathcal{Q}^{ \pm}\left(\partial_{\varphi}, \frac{1}{2}\right) \mathcal{V}^{k}( \pm \pi, t)=0
$$

for $j=1,2$ and all $t \in(0, T)$.

Let us prove now that the linear mapping of problem (4.16) is an isomorphism from

$$
\left\{\mathcal{V} \in H^{\ell+1}(\Upsilon)^{2}: \mathcal{V} \text { verifies the orthogonality conditions }(7.10)\right\}
$$

onto the linear subspace

$$
\left\{\left(\mathcal{F}, \mathcal{G}^{ \pm}\right) \in R^{\ell} H(\Upsilon):\left(\mathcal{F}, \mathcal{G}^{ \pm}\right) \text {verifies the compability conditions }(4.17)\right\} .
$$

Indeed, the mapping is an epimorphism in view of Lemma 4.4. It is a monomorphism since, owing to definitions (7.4), (7.6) and the assumption det $\mathfrak{M} \neq 0$, any element of the kernel $b_{1} \mathcal{V}_{1}(\varphi)+b_{2} \mathcal{V}_{2}(\varphi)$ subject to the orthogonality condition (7.10) is zero.

Therefore, problem (7.8)-(7.9) leads to an homogeneous Volterra equation of second kind on $\Upsilon$, which admits only the trivial solution. Thus $\mathcal{V}^{k}=0$ and we have a contradiction which completes the proof of theorem.

\subsection{An interface crack}

In this section, we are going to construct explicitly a singularity containing an infinite logarithmic packet for the case when the relaxation kernel $B(\varphi)$ takes two distinct values in the lower half-plane $\varphi \in(-\pi, 0)$ and the upper half-plane $\varphi \in(0, \pi)$, corresponding to two different isotropic materials. Stricto sensu, this case 
does not satisfy the assumptions adopted in the paper, since it is supposed in (1.9) that $\varphi \mapsto B(\varphi)$ is smooth. Nevertheless all our results can be extended to the new framework of a piecewise constant relaxation kernel, see Remark 2.3. As a fundamental ingredient, we use a formula giving the imaginary shift $i \nu$ in the spectrum (4.13) for an interface crack.

Lemma 7.2. Let $\mathcal{O}$ be the tip of the interface crack $\left\{\left(x_{1}, 0\right): x_{1}<0\right\}$ between two isotropic materials with Lamé constants $\left(\lambda_{1}, \mu_{1}\right)$ and $\left(\lambda_{2}, \mu_{2}\right)$. Each material fills a half-space, $x_{2}<0$ and $x_{2}>0$, respectively. The spectrum of the Mellin operator pencil associated with this transmission problem at the point $\mathcal{O}$ has the form (4.13) with

$$
\nu=\frac{1}{2 \pi} \ln \left(\frac{\lambda_{1}+\mu_{1}}{\lambda_{2}+\mu_{2}} \cdot \frac{\lambda_{2}\left(\mu_{1}+\mu_{2}\right)+3 \mu_{1} \mu_{2}+\mu_{2}^{2}}{\lambda_{1}\left(\mu_{1}+\mu_{2}\right)+3 \mu_{1} \mu_{2}+\mu_{1}^{2}}\right) .
$$

Proof. Formula (7.11) is deduced from explicit calculations made in [12], Section 3.3 (we refer also to [13] for this type of formulae): we find in (3.27) loc. cit. that

$$
\nu=\frac{1}{2 \pi} \ln \frac{\gamma_{5}-\gamma_{6}}{\gamma_{5}+\gamma_{6}}
$$

Going backward in the paper, we find formulas for $\gamma_{5}$ and $\gamma_{6}$ as functions of $\mu_{1}, \mu_{2}$ and the Poisson ratios $\sigma_{1}$, $\sigma_{2}$. And we arrive at

$$
\nu=\frac{1}{2 \pi} \ln \frac{\mu_{1}\left(3-4 \sigma_{2}\right)+\mu_{2}}{\mu_{1}+\mu_{2}\left(3-4 \sigma_{1}\right)} .
$$

Replacing $\sigma_{k}$ by its expression with respect to $\lambda_{k}$ and $\mu_{k}$ :

$$
\sigma_{k}=\frac{\lambda_{k}}{2\left(\lambda_{k}+\mu_{k}\right)}
$$

we find (7.11).

Remark 7.3. From formula (7.12) we easily deduce that $\nu=0$, i.e., $\mu_{1}\left(3-4 \sigma_{2}\right)+\mu_{2}=\mu_{1}+\mu_{2}\left(3-4 \sigma_{1}\right)$ if and only if the Dundurs relation is satisfied

$$
\frac{\mu_{1}}{\mu_{2}}=\frac{1-2 \sigma_{1}}{1-2 \sigma_{2}}
$$

See also [12], (3.25).

Let us consider the situation where:

- the Hooke matrix $A$ is that of an isotropic material of Lamé constants $(\lambda, \mu)$;

- the relaxation kernel $\varphi \mapsto B(\varphi)$ (still independent of time) is defined for $\varphi<0$ (i.e., $x_{2}<0$ ) by the matrix $B_{-}$of Lamé constants $\left(\lambda_{-}, \mu_{-}\right)$and for $\varphi>0$ (i.e., $\left.x_{2}>0\right)$ by the matrix $B_{+}$of Lamé constants $\left(\lambda_{+}, \mu_{+}\right)$.

$A$ and $B_{ \pm}$are of the form (1.14) with $\lambda, \mu$ and $\lambda_{ \pm}, \mu_{ \pm}$, respectively.

We are going to construct an infinite logarithmic packet for the creep problem associated with $A$ and $B \equiv B_{ \pm}$ by a comparison with the singularities of the interface problem of purely elastic material law $A+\varepsilon B_{ \pm}$where $\varepsilon$ is a small parameter.

Lemma 7.4. Let $i \nu(\varepsilon)$ be the imaginary shift of the spectrum associated with $A+\varepsilon B_{ \pm}$. Then

$$
\nu(\varepsilon)=\frac{1}{2 \pi} \ln \kappa(\varepsilon)
$$

where $\varepsilon \mapsto \kappa(\varepsilon)$ is analytic in a neighborhood of 0 . The Taylor expansion of $\kappa$ at 0 starts with

$$
\kappa(\varepsilon)=1+\varepsilon \kappa_{1}+\mathcal{O}\left(\varepsilon^{2}\right), \quad \varepsilon \rightarrow 0,
$$


with

$$
\kappa_{1}=\frac{\mu}{(\lambda+\mu)(\lambda+2 \mu)}\left(\lambda_{+}+\mu_{+}-\lambda_{-}-\mu_{-}\right) .
$$

Proof. We use formula (7.11) with

$$
\lambda_{1}=\lambda+\varepsilon \lambda_{-}, \quad \mu_{1}=\mu+\varepsilon \mu_{-}, \quad \lambda_{2}=\lambda+\varepsilon \lambda_{+}, \quad \mu_{2}=\mu+\varepsilon \mu_{+} .
$$

We find for $\kappa(\varepsilon)$ a rational fraction with numerator and denominator of degree 3 in $\varepsilon$ and tending to 1 as $\varepsilon \rightarrow 0$. Computing $\kappa_{1}$ yields (7.16).

We assume that

$$
\lambda_{+}+\mu_{+} \neq \lambda_{-}+\mu_{-},
$$

which ensures that $\nu(\varepsilon) \neq 0$ for $0<\varepsilon<\varepsilon_{0}$ with $\varepsilon_{0}>0$. Then the "first" singularities of the interface crack $A+\varepsilon B_{ \pm}$have the form of

$$
V(\varepsilon ; x)=r^{\frac{1}{2}+i \nu(\varepsilon)} \mathcal{V}(\varepsilon ; \varphi)=r^{\frac{1}{2}} \mathrm{e}^{i \nu(\varepsilon) \ln r} \mathcal{V}(\varepsilon ; \varphi),
$$

and their complex conjugates. It is possible to choose $\varepsilon \mapsto \mathcal{V}(\varepsilon ; \varphi)$ so that the dependence is analytic and $\mathcal{V}(0 ; \varphi) \not \equiv 0$. Composing $\varepsilon$-expansions of $\nu(7.14)-(7.15)$ and $\mathcal{V}$ with series expansion of the exponential function we find that

$$
V(\varepsilon ; x)=\sum_{k=0}^{\infty} \varepsilon^{k} V^{k}(x) \quad \text { with } \quad V^{k}(x)=\left(\frac{i \kappa_{1}}{2 \pi}\right)^{k} r^{\frac{1}{2}} \frac{(\ln r)^{k}}{k !} \mathcal{V}(0 ; \varphi)+\sum_{j=0}^{k-1} r^{\frac{1}{2}}(\ln r)^{j} \mathcal{V}^{k j}(\varphi)
$$

Since $\left(A+\varepsilon B_{ \pm}\right) V(\varepsilon ; x) \equiv 0$, we find that the terms $V^{k}$ of the series (7.18) satisfy

$$
\left\{L, N^{ \pm}\right\} V^{k}=-\left\{P, Q^{ \pm}\right\} V^{k-1} \quad \forall k \in \mathbb{N} .
$$

We come back to the creep problem by the following trick: we set

$$
u^{k}(x, t)=\frac{t^{k}}{k !} V^{k}(x) .
$$

Thanks to (7.19), we check that the series $u^{k}$ solves the instantaneous problems $\left\{L, N^{ \pm}\right\} u^{k}=\left\{f^{k}, g^{k}\right\}$ with $\left\{f^{k}, g^{k}\right\}$ given by (3.19). Therefore the associated series (3.18) $\sum_{k} u^{k}:=u(x, t)$ solves the homogeneous creep problem in an infinite cracked domain. Combining (7.18) with (7.20) gives finally

$$
u(x, t)=\sum_{k=0}^{\infty} \frac{t^{k}}{k !}\left[\left(\frac{i \kappa_{1}}{2 \pi}\right)^{k} r^{\frac{1}{2}} \frac{(\ln r)^{k}}{k !} \mathcal{V}(0 ; \varphi)+\sum_{j=0}^{k-1} r^{\frac{1}{2}}(\ln r)^{j} \mathcal{V}^{k j}(\varphi)\right]
$$

Considering $\chi^{\mathfrak{i}}(x) u\left(x_{\mathfrak{i}}, t\right)$ produces a solution of the creep problem with smooth and flat right hand side, containing an infinite logarithmic packet.

Remark 7.5. a) By extension, the proof of the last formula shows that logarithmic packets also appear when the set (4.13) does not coincide with $\frac{1}{2} \mathbb{Z}$, i.e., the exponents of power solutions have non-trivial imaginary parts $\nu(t)$.

b) Since logarithmic packets are present for aging materials if the Dundurs relation (7.13) is violated, these packets can appear at a crack on the interface of an isotropic aging material provided that the lower and upper parts of the material on both sides of the crack are of different age. 
Acknowledgements. The paper was prepared during visits of S.A. Nazarov at the Institute Élie Cartan in Nancy and at IRMAR in Rennes. The research is supported by INRIA in the framework of a grant from Institut franco-russe A.M. Liapunov d'Informatique et de Mathématiques Appliquées and by the Russian Foundation for Basic Research (grant 03-01-00835).

\section{REFERENCES}

[1] M.S. Agranovich and M.I. Vishik, Elliptic problems with the parameter and parabolic problems of general type. Uspekhi Mat. Nauk 19 (1963) 53-161 (English transl.: Russ. Math. Surv. 19 (1964) 53-157).

[2] N.Kh. Arutyunyan and V.B. Kolmanovskii, The theory of creeping heterogeneous bodies. Nauka, Moscow (1983) 336.

[3] N.Kh. Arutyunyan, S.A. Nazarov and B.A. Shoikhet, Bounds and the asymptote of the stress-strain state of a threedimensional body with a crack in elasticity theory and creep theory. Dokl. Akad. Nauk SSSR 266 (1982) 1361-1366 (English transl.: Sov. Phys. Dokl. 27 (1982) 817-819).

[4] N.Kh. Arutyunyan, A.D. Drozdov and V.E. Naumov, Mechanics of growing visco-elasto-plastic bodies. Nauka, Moscow (1987) 472 .

[5] C. Atkinson and J.P. Bourne, Stress singularities in viscoelastic media. Q. J. Mech. Appl. Math. 42 (1989) 385-412.

[6] C. Atkinson and J.P. Bourne, Stress singularities in angular sectors of viscoelastic media. Int. J. Eng. Sci. 28 (1990) 615-650.

[7] J.P. Bourne and C. Atkinson, Stress singularities in viscoelastic media. II. Plane-strain stress singularities at corners. IMA J. Appl. Math. 44 (1990) 163-180.

[8] M. Costabel and M. Dauge, Construction of corner singularities for Agmon-Douglis-Nirenberg elliptic systems. Math. Nachr. 162 (1993) 209-237.

[9] M. Costabel and M. Dauge, Crack singularities for general elliptic systems. Math. Nachr. 235 (2002) 29-49.

[10] M. Costabel, M. Dauge and R. Duduchava, Asymptotics without logarithmic terms for crack problems. Comm. Partial Differential Equations 28 (2003) 869-926.

[11] R. Duduchava and W.L. Wendland, The Wiener-Hopf method for systems of pseudodifferential equations with an application to crack problems. Integr. Equat. Oper. Th. 23 (1995) 294-335.

[12] R. Duduchava, A.M. Sändig and W.L. Wendland, Interface cracks in anisotropic composites. Math. Method. Appl. Sci. 22 (1999) 1413-1446.

[13] J. Dundurs, Effect of elastic constants on stress in composite under plane deformations. J. Compos. Mater. 1 (1967) 310.

[14] G. Gripenberg, S.-O. Londen and O. Staffans, Volterra Integral and Functional equations. Cambridge Univ. Press, Cambridge (1990).

[15] V.A. Kondratiev, Boundary problems for elliptic equations in domains with conical or angular points. Trudy Moskov. Mat. Obshch. 16 (1967) 209-292 (English transl.: Trans. Moscow Math. Soc. 16 (1967) 227-313).

[16] V.A. Kondratiev and O.A. Oleinik, Boundary-value problems for the system of elasticity theory in unbounded domains. Korn's inequalities. Uspehi Mat. Nauk 43 (1988) 55-98 (English transl.: Russ. Math. Surv. 43 (1988) 65-119).

[17] V.A. Kozlov, V.G. Maz'ya and J. Rossmann, Elliptic boundary value problems in domains with point singularities. Amer. Math. Soc., Providence (1997).

[18] M.A. Krasnosel'skii, G.M. Vainikko and P.P. Zabreiko, Approximate solutions to integral equations. Nauka, Moscow (1969) 455.

[19] V.G. Maz'ya and B.A. Plamenevskii, Weighted spaces with nonhomogeneous norms and boundary value problems in domains with conical points. In: Elliptische Differentialgleichungen (Meeting in Rostock, 1977), Wilhelm-Pieck-Univ., Rostock (1978) 161-189 (English transl.: Amer. Math. Soc. Transl. Ser. 2123 (1984) 89-107).

[20] V.G. Maz'ya and B.A. Plamenevskii, The coefficients in the asymptotics of solutions of elliptic boundary value problems in domains with conical points. Math. Nachr. 76 (1997) 29-60 (English transl.: Amer. Math. Soc. Transl. Ser. 2123 (1984) $57-88)$.

[21] S.E. Mikhailov, Singularities of stresses in a plane hereditarily-elastic aging solid with corner points. Mech. Solids (Izv. AN SSSR. MTT) 19 (1984) 126-139.

[22] S.E. Mikhailov, Singular stress behavior in a bonded hereditarily-elastic aging wedge. Part. I: Problem statement and degenerate case. Math. Method. Appl. Sci. 20 (1997) 13-30.

[23] S.E. Mikhailov, Singular stress behavior in a bonded hereditarily-elastic aging wedge. Part. II: General heredity. Math. Method. Appl. Sci. 20 (1997) 31-45.

[24] S.A. Nazarov, Vishik-Lyusternik method for elliptic boundary-value problems in regions with conical points. 1. The problem in a cone. Sibirsk. Mat. Zh. 22 (1981) 142-163 (English transl.: Siberian Math. J. 22 (1982) 594-611).

[25] S.A. Nazarov, Weight functions and invariant integrals. Vychisl. Mekh. Deform. Tverd. Tela. 1 (1990) 17-31. (Russian)

[26] S.A. Nazarov, Self-adjoint boundary value problems. The polynomial property and formal positive operators. St.-Petersburg Univ., Probl. Mat. Anal. 16 (1997) 167-192. (Russian)

[27] S.A. Nazarov, The interface crack in anisotropic bodies. Stress singularities and invariant integrals. Prikl. Mat. Mekh. 62 (1998) 489-502 (English transl.: J. Appl. Math. Mech. 62 (1998) 453-464). 
[28] S.A. Nazarov, The polynomial property of self-adjoint elliptic boundary-value problems and the algebraic description of their attributes. Uspekhi mat. nauk 54 (1999) 77-142 (English transl.: Russ. Math. Surv. 54 (1999) 947-1014).

[29] S.A. Nazarov and B.A. Shoikhet, Asymptotic behavior of the solution of a certain integro-differential equation near an angular point of the boundary. Mat. Zametki. 33 (1983) 583-594 (English transl.: Math. Notes 33 (1983) 300-306).

[30] S.A. Nazarov and B.A. Plamenevskii, Neumann problem for selfadjoint elliptic systems in a domain with piecewise smooth boundary. Trudy Leningrad. Mat. Obshch. 1 (1990) 174-211 (English transl.: Amer. Math. Soc. Transl. Ser. 2 155 (1993) 169-206).

[31] S.A. Nazarov and B.A. Plamenevsky, Elliptic problems in domains with piecewise smooth boundaries. Walter de Gruyter, Berlin, New York (1994) 525.

[32] S.A. Nazarov, L.P. Trapeznikov and B.A. Shoikhet, On the correspondence principle in the plane creep problem of aging homogeneous media with developing slits and cavities. Prikl. Mat. Mekh. 51 (1987) 504-512 (English transl.: J. Appl. Math. Mech. 51 (1987) 392-399).

[33] J. Nečas, Les méthodes directes en théorie des équations elliptiques. Masson-Academia, Paris-Prague (1967).

[34] A.C. Pipkin, Lectures on viscoelasticity theory. Springer, NY (1972) 180.

[35] G.S. Vardanyan and V.D. Sheremet, On certain theorems in the plane problem of the creep theory. Izvestia AN Arm. SSR. Mechanics 4 (1973) 60-76.

[36] V.P. Zhuravlev, S.A. Nazarov and B.A. Shoikhet, Asymptotics of the stress-strain state near the tip of a crack in an inhomogeneously aging bodies. Dokl. Akad. Nauk Armenian SSR 74 (1982) 26-29. (Russian)

[37] V.P. Zhuravlev, S.A. Nazarov and B.A. Shoikhet, Asymptotics near the tip of a crack of the state of stress and strain of inhomogeneously aging bodies. Prikl. Mat. Mekh. 47 (1983) 200-208 (English transl.: J. Appl. Math. Mech. 47 (1984) 162170).

To access this journal online: www.edpsciences.org 